\title{
WestVirginiaUniversity
}

THE RESEARCH REPOSITORY @ WVU

Graduate Theses, Dissertations, and Problem Reports

2017

\section{Exploring Staff Turnover in a Large-Scale EBT Implementation Initiative}

Laurel A. Brabson

Follow this and additional works at: https://researchrepository.wvu.edu/etd

\section{Recommended Citation}

Brabson, Laurel A., "Exploring Staff Turnover in a Large-Scale EBT Implementation Initiative" (2017). Graduate Theses, Dissertations, and Problem Reports. 5249.

https://researchrepository.wvu.edu/etd/5249

This Thesis is protected by copyright and/or related rights. It has been brought to you by the The Research Repository @ WVU with permission from the rights-holder(s). You are free to use this Thesis in any way that is permitted by the copyright and related rights legislation that applies to your use. For other uses you must obtain permission from the rights-holder(s) directly, unless additional rights are indicated by a Creative Commons license in the record and/ or on the work itself. This Thesis has been accepted for inclusion in WVU Graduate Theses, Dissertations, and Problem Reports collection by an authorized administrator of The Research Repository @ WVU. For more information, please contact researchrepository@mail.wvu.edu. 
Exploring Staff Turnover in a Large-Scale EBT Implementation Initiative

Laurel A. Brabson, B.A.

Thesis defense submitted to the Eberly College of Arts and Sciences

at West Virginia University in partial fulfillment of the requirements

for the degree of Master of Science in Psychology

Amy D. Herschell, Ph.D., Chair Amy Fiske, Ph.D.

Nicholas Turiano, Ph.D.

Department of Psychology

Morgantown, West Virginia

2017

Keywords: Behavioral Health, Turnover, Evidence-Based Treatment, Predictors 


\section{Abstract \\ Exploring Staff Turnover in a Large-Scale EBT Implementation Initiative Laurel A. Brabson}

Staff turnover in the behavioral health field is a substantial and chronic problem with implications for both agencies and clients (Ben-Dror, 1994). Increased focus on the implementation of evidence-based treatments (EBT) has further highlighted the problems associated with turnover, as EBT trainings are particularly costly and time-consuming for clinicians and their agencies (Cook, Biyanova, \& Coyne, 2009). The current study examined rates and predictors of turnover within an EBT implementation initiative designed to assess the effectiveness of three different training models. Data was collected from 110 families, 100 clinicians, 50 supervisors, and 50 administrators involved in the state-wide implementation of Parent-Child Interaction Therapy (PCIT). Overall, rates of staff turnover (8\% at 12 months, 30\% at 24 months) were less than typically reported by community behavioral health organizations (50\% or greater at 12 months). In addition, organizational climate was a significant predictor of supervisor and administrator turnover, with different rates of turnover noted across different training conditions. Evidence also suggested that clinician turnover may be associated with poor client outcomes, although for a limited proportion of families. Implications for behavioral health agencies and future directions for research are discussed. 


\section{Table of Contents}

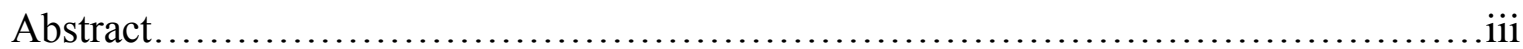

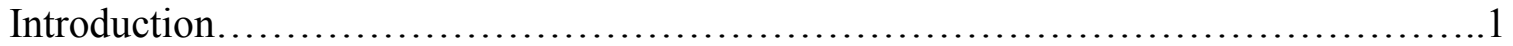

Hypothesis...........................................................20

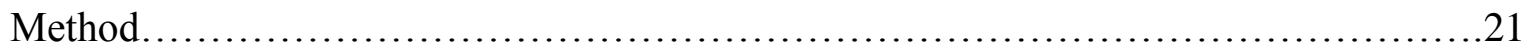

Participants............................................................21

Procedures.........................................................22

Measures...............................................................23

Definition of Turnover..................................................... 27

Data Analyses...................................................... 27

Results................................................................. 28

Sample Characteristics................................................... 28

Aim 1: Rates of Turnover.............................................. 30

Aim 2: Predictors of Turnover.......................................... 31

Aim 3: Therapeutic Outcomes.......................................... 35

Discussion.............................................................. 38

References.............................................................53

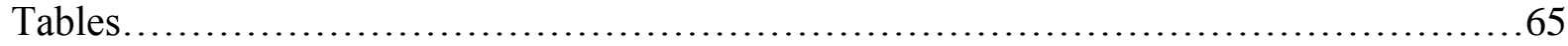

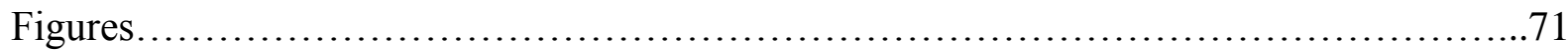

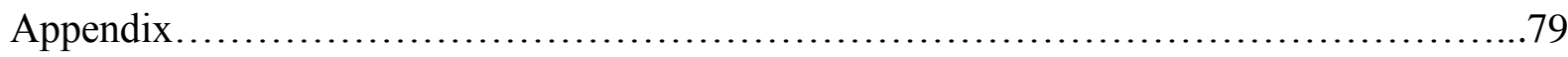




\section{Introduction}

Turnover, broadly defined, is the separation of an employee from an establishment (Bureau of Labor Statistics, n.d.). Rates of turnover vary across professions and thus are more problematic in some industries than others. For instance, physicians, nurses, and teachers are generally thought of as having problematic annual turnover rates which are estimated at $7 \%$, 14\%, and 11\%, respectively (Flatt, 2014; Gray \& Phillips, 1994; Ingersoll, 2001). Even more problematic are annual turnover rates for behavioral health staff, which are consistently reported to be approximately 50\% or greater (e.g., Ben-Dror, 1994; Bjorklund, Monroe-DeVita, Reed, Toulon, \& Morse, 2009; Selden, 2010).

Numerous problems arise from staff turnover in behavioral health settings. Research has demonstrated negative effects of turnover on the organization as a whole, as well as on other staff members within the organization. Specifically, turnover has been shown to have a negative impact on staff morale, productivity, and organizational effectiveness (Iglehart, 1990). Turnover also results in added financial burden, as the organization must pay to recruit and train new staff members (Ben-Dror, 1994). These financial burdens are exacerbated when the organization is involved in implementing evidence-based treatments (EBTs) due to the increased cost of training and fidelity monitoring often associated with EBTs (Bjorklund et al., 2009). Perhaps more problematic, turnover has been hypothesized to result in significant disruption of the therapeutic relationship and in overall service delivery for clients (Bjorklund et al., 2009), which may hinder therapeutic progress and outcomes (Adair et al., 2005).

Given its problematic nature, research has been aimed at identifying factors that can predict turnover within the behavioral health field. Demographic factors that are predictive of turnover include age and education (Ben-Dror, 1994); and job level and tenure with the organization (Blankertz \& Robinson, 1997). Organizational factors that are predictive of 
turnover include organizational culture (i.e., norms and expectations for how things are done; Aarons \& Sawitzky, 2006; Glisson et al., 2008), organizational climate (i.e., employee attitudes toward and perceptions of the work environment; Aarons \& Sawitzky, 2006; Glisson et al., 2008); amount and quality of clinical supervision (Kim \& Lee, 2009; Knudsen, Ducharme, \& Roman, 2008); amount of paperwork (McGowan, Auerbach, \& Strolin-Goltzman, 2009), and pay rate (Ben-Dror, 1994). A third type of variable related to turnover results from the interaction between demographic and organizational variables, and includes factors such as emotional exhaustion (Aarons, Fettes, Flores, \& Sommerfeld, 2009; Knudsen et al., 2008), role stress (Kim \& Stoner, 2008), social support (Kim \& Stoner, 2008), and perceived job autonomy (Knudsen et al., 2008).

Staff turnover is a complex phenomenon influenced by numerous diverse factors, including the context in which it is studied. The present study investigated factors that influenced staff turnover within a statewide initiative to implement Parent-Child Interaction Therapy (PCIT), an EBT for families of young children with disruptive behavior disorders. The purposes of the current study were to: (a) describe rates of across agency clinicians, supervisors, and administrators; (b) identify predictors of turnover specific to this statewide implementation initiative; and (c) examine the potential relation between clinician turnover and client outcomes. The following is a review of the definition of turnover, the significance of turnover for behavioral health treatment, and the numerous variables that have been associated with turnover.

\section{Definition of Turnover}

Although the concept of turnover may seem straightforward, researchers have used different definitions across studies. For example, some studies have examined turnover intent (i.e., "conscious and deliberate willfulness to leave [an] organization;” Tett \& Meyer, 1993, p. 
262) as an outcome variable and a proxy for actual turnover (e.g., Kim \& Stoner, 2008; Knudsen et al., 2008). Researchers have justified the use of turnover intent based on the argument that “the best single predictor of an individual's behavior [is] a measure of his intention to perform that behavior" (Fishbein \& Ajzen, 1975, p. 369). Although research has shown that turnover intentions do predict actual turnover (Steel \& Ovalle, 1984; Tett \& Meyer, 1993), additional research has demonstrated that a multitude of other factors contribute to an employee ultimately leaving an organization. Thus, turnover intent should remain a predictor of, rather than a proxy for, actual turnover.

Although the broad definition of turnover applies to any employee who leaves an organization, a distinction must be made between voluntary and involuntary (i.e., employees who were fired) turnover. To some degree, involuntary turnover can be beneficial to the organization, as it provides opportunities to replace employees who are underperforming with new employees who have the potential to perform better. Taking this into account, it is important to note that some studies focus exclusively on voluntary turnover (e.g., Ben-Dror, 1994; Wright \& Cropanzano, 1998) and some combine voluntary and involuntary turnover (e.g., Aarons \& Sawitzky, 2006; Kolko et al., 2012; Rollins, Salyers, Tsai, \& Lydick, 2010). Employees who leave voluntarily versus involuntarily are not a homogenous group and inherently have different reasons for leaving their role; therefore, combining them into one group confounds the process of identifying predictors of turnover.

One final complication in the definition of turnover is the use of different time periods in which turnover is measured. Often, the time period is determined by the amount of time devoted to data collection within the research study, but can also be influenced by the administrative practices within the organization. For instance, studies have used 12 months, (e.g., Glisson et al., 
2008), 24 months (e.g., Woltmann et al., 2008), 2.5 years (Sheidow, Schoenwald, Wagner, Allred, \& Burns, 2007), and methods of converting longer time periods into 12-month estimates (e.g., Rollins, Salyers, Tsai, \& Lydick, 2010). The use of different time periods can cause difficulty when comparing rates of turnover across studies.

\section{Importance of Turnover}

Prevalence estimates indicate that roughly half of the population will meet criteria for a mental illness at some point in their lifetimes (Kessler et al., 2005; Kessler \& Wang, 2008). Findings also indicate that the majority of mental illnesses first commence in childhood and adolescence, with about 50\% presenting before age 14 (Catania, Hetrick, Newman, \& Purcell, 2011). It is crucial that individuals with mental illness have access to effective therapeutic interventions. This is especially true for children, as evidence has shown that earlier age of onset is associated with a more chronic and severe condition if untreated (Kessler \& Wang, 2008).

Unfortunately, national estimates have also shown that only $47 \%$ of children receive clinically-indicated services for which they are referred (Dougherty, Schiff, \& Mangione-Smith, 2011). This difficulty in receiving care has been primarily attributed to a general lack of highquality behavioral health services, especially for children (Oppenheim et al., 2016), which is exacerbated by high rates of clinician turnover. When children are able to receive services, it is possible that they will experience a disruption in the therapeutic relationship as a result of clinician turnover (Strolin-Goltzman, Kollar, \& Trinkle, 2010). Although many researchers have hypothesized that clinician turnover may negatively impact client outcomes as a result of the ruptured therapeutic relationship (e.g., Albizu-García, Ríos, Juarbe, \& Alegría, 2004; Kim \& Stoner, 2008; Mor Barak et al., 2001; Sheidow et al., 2007; Woltmann et al., 2008), evidence is mixed. Once study found very little effect of clinician turnover on outcomes for youth receiving 
substance use treatment (Garner, Funk, \& Hunter, 2013), although other studies have shown worse outcomes for youth in foster care (Strolin-Goltzman et al., 2010) and for adults with chronic pain (Williams \& Potts, 2010) who were impacted by clinician turnover.

Although additional research is needed to understand the degree to which turnover directly impacts client outcomes, evidence has shown that turnover can place added burdens on remaining staff members, causing lower morale (Iglehart, 1990) and ultimately a deterioration in service quality (Maslach \& Jackson, 1981). Thus, evidence would suggest that even when children are able to access behavioral health services, there is a possibility that they may not be receiving the highest quality care possible.

One strategy that has been identified as both a professional (i.e., by the American Psychological Association; American Psychological Association, 2005) and national priority (President's New Freedom Commission on Mental Health, 2003) for improving the quality of behavioral health services is the increased implementation of evidence-based treatments (EBTs). While this has prompted numerous effectiveness trials and implementation initiatives, it is ultimately incumbent upon the workforce to successfully learn and sustain the interventions. Turnover in agencies implementing EBTs can be especially problematic due to three core features common to most EBTs: (a) intensive training, (b) highly structured/manualized protocols, and (c) fidelity monitoring (Aarons, Sommerfeld, Hecht, Silovsky, \& Chaffin, 2009). These features often require additional resources and funding compared with other nonevidence-based interventions or treatment as usual. For implementation efforts to be successful and sustainable, turnover of staff trained in EBTs must be minimized.

Given the problems associated with turnover in the behavioral health field, researchers have focused on identifying factors that are associated with turnover. These efforts are critical to 
identifying employees at risk for turnover, for creating organizational interventions that may reduce turnover, and for ultimately creating a more stable, high-quality, and effective behavioral health workforce.

\section{Limitations}

Prior to reviewing predictor variables associated with turnover, there are a number of limitations within the turnover literature that warrant discussion, including: the use of turnover intent as an outcome in place of actual turnover, the lack of a clear and/or consistent operational definition of turnover across studies, and the lack of a consistent time period for measuring turnover across studies. These limitations, in addition to those discussed below, result in the need for some degree of caution when interpreting and comparing results of turnover studies.

Methodology. When reviewing the literature to identify possible predictors of turnover, it is important to consider the type of methodology used. Turnover research lends itself to both qualitative and quantitative designs. While this is not inherently a limitation, and can even act as a strength in some cases, it is important to understand the difference in types of information gained from qualitative versus quantitative research.

Some studies have identified factors contributing to turnover using strictly qualitative methods. For example, one study used focus groups and qualitative analyses to identify factors contributing uniquely to employee turnover and retention based on the perspectives of child welfare professionals (Ellett, Ellis, Westbrook, \& Dews, 2007). While this type of study provides a wealth of detailed information, statistical analyses designed to assess the predictive strength of each factor cannot be used. Additionally, this qualitative data is based on the experiences of a specific type of professional and should not be generalized to other professionals. 
Other studies have employed mixed-method designs in order to reap the benefits of both qualitative and quantitative analyses. For example, Woltmann and colleagues (2008) collected quantitative data to determine the rate of staff turnover in a wide-scale EBT implementation initiative. They also collected qualitative data to understand staff perspectives regarding the role turnover played in the overall success and sustainability of the initiative (Woltmann et al., 2008). While this mixed-method study provided important information regarding how turnover can impact implementation initiatives, it did not provide any information on specific predictors of staff turnover in behavioral health settings.

Samples. Perhaps most relevant to the current study is the dearth of literature regarding turnover of behavioral health staff specifically. For example, previous studies have examined turnover in samples of retail and insurance salespeople (Allen, Shore, \& Griffeth, 2003), unspecified hospital employees (Cropanzano, Rupp, \& Byrne, 2003), and unspecified workers employed at least part-time (Lambert, Hogan, \& Barton, 2001), among others. While this general information can be helpful in identifying potential variables of interest for continued study, different stressors are associated with different types of work and those specific to behavioral health workers may not be endorsed by the general workforce.

With the recent nationwide focus on implementing EBTs, there seems to have been an increased interest in turnover within the field of behavioral health. However, the fact remains that research on behavioral health staff turnover lags behind that within similar industries, including substance abuse treatment (e.g., Knudsen, Ducharme, \& Roman, 2006; Knudsen et al., 2008; McNulty, Oser, \& Johnson, 2007) and child welfare (e.g, Aarons \& Palinkas, 2007; Ellett et al., 2007; McGowan, Auerbach, \& Strolin-Goltzman, 2009; Mor Barak, Nissly, \& Levin, 2001). As the success and sustainability of EBT implementation initiatives is contingent upon a 
stable workforce, further research is needed to investigate factors underlying turnover for behavioral health staff specifically.

\section{Predictors of Turnover}

It is apparent that turnover research is complicated by numerous limitations.

Unfortunately, it is further complicated by the actual variables of interest for a few reasons. First, many of the variables that have been associated with turnover are multidimensional, encompassing several distinct constructs. In some studies, these multidimensional variables have been disaggregated and only a few of the underlying constructs have been studied (detailed examples are provided below). This disaggregation makes comparisons across studies challenging. Second, some studies have identified specific variables as direct predictors of turnover, whereas others have found the same variables to have a mediational or indirect effect on turnover. These complexities result in a somewhat disorganized model of turnover.

In order to understand which variables might be important to assess within the current study, the literature on both general workforce turnover and behavioral health workforce literate was reviewed. Figure 1 organizes the variables identified in the literature based on the following categories: demographic predictors of general workforce turnover, organizational predictors of general workforce turnover, demographic predictors of behavioral health turnover, and organizational predictors of behavioral health turnover. Please note that this figure only includes studies that examined actual turnover; those studies that measured turnover intent as the outcome variable were not included. What follows is a summary of the literature on predictors of turnover, in an effort to further delineate these complexities and underscore variables of interest specific to research on turnover in behavioral health settings. 
Demographic predictors of behavioral health turnover. Perhaps the least complex of the variables associated with turnover are the demographic variables. In one of the first studies examining predictors of turnover specifically for behavioral health staff, Ben-Dror (1994) identified that age and salary were negatively associated with turnover for employees of a community behavioral health residential center. Additionally, there was an association between education and turnover, whereby individuals with a Masters-level education were more likely to leave the organization than individuals with either less or more education (Ben-Dror, 1994). Findings from this study also indicated that an employee's decision to leave an organization was most influenced by salary (Ben-Dror, 1994). A second study examining staff turnover during the implementation of an EBT for adolescents also identified salary as a significant predictor of turnover (Sheidow et al., 2007). It is interesting to note that in these two studies, salary was measured as the actual numerical value as opposed to a more subjective indicator of the employee's satisfaction with his/her salary. However, another recent study using qualitative methods noted that $41 \%$ of participants $(N=29$ clinicians at outpatient behavioral health clinics) who left their agencies reported that financial reasons impacted their decision to leave (Beidas et al., 2015). Thus, regardless of how it is measured, salary seems to be an important factor in staff turnover.

Organizational predictors of behavioral health turnover. Organizational factors associated with behavioral health turnover are more complex than demographic factors. This is largely because the organizational factors that have been studied thus far have consisted almost entirely of multidimensional variables. The sole exceptions are service structure (i.e., the manner in which adult and children's services are organized within the agency) and organization size. Service structure was identified as a predictor of turnover in a large-scale survey of a nationwide 
sample of behavioral health clinics (Glisson, Schoenwald, et al., 2008). Results of this study indicated that turnover rates were found to be higher in agencies with separate children's service units compared with units that served both children and adults (Glisson, Schoenwald, et al., 2008). Additionally, organization size was implicated as a predictor of turnover in recent survey examining factors that influenced turnover in community behavioral health settings across Ohio (Bukach, Ejaz, Dawson, \& Gitter, 2015). Specifically, results indicated that smaller organizations tended to have lower rates of turnover than larger organizations (Bukach et al., 2015).

Organizational culture. Organizational culture is defined as the "normative beliefs and shared behavioral expectations" regarding how things are done in a work unit (Glisson \& James, 2002, p. 770). This is a broad definition and has been measured in a variety of ways within turnover literature. For example, in a study examining the effects of organizational culture, climate, and work attitudes on turnover within a sample of behavioral health case managers, organizational culture was measured dimensionally as constructive or defensive, with defensive cultures considered more problematic (Aarons \& Sawitzky, 2006). In another study examining correlates of turnover in a large-scale survey of behavioral health clinics, organizational culture was defined categorically as either rigid, proficient, or resistant, with both rigid and resistant cultures considered problematic (Glisson, Schoenwald, et al., 2008). Although the majority of studies have categorized agencies based on different types of cultures (Aarons \& Sawitzky, 2006; Glisson, Schoenwald, et al., 2008; Glisson, Williams, Hemmelgarn, Proctor, \& Green, 2016), organizational culture has also been measured as a continuous variable related to an individual's positive or negative perceptions of the day-to-day practices and structure within the organization (Lehman, Greener, \& Simpson, 2002). While research demonstrated that negative 
organizational cultures were associated with higher rates of turnover, the use of different definitions make it difficult to parse apart which specific facets of negative culture may have been driving turnover.

Organizational climate. Methods for defining and measuring organizational climate are equally complicated. Organizational climate has been defined as employee perceptions of the overall work environment (Pritchard \& Karasick, 1973). This broad definition has led to various interpretations and measures of organizational climate within research on turnover. For example, Sheidow and colleagues (2007) included measures of "fairness, role clarity, role overload, role conflict, cooperation, growth and advancement, job satisfaction, emotional exhaustion, personal accomplishment, and depersonalization" (p. 49) as indicators of organizational climate. Aarons and Sawitzky (2006) only assessed emotional exhaustion and depersonalization using Likert-type scales in their overall measure of organizational climate. Glisson and colleagues (2008) took a different approach by categorizing organizational climate as engaged, functional, or stressful, with stressful climates considered more negative and problematic. Despite these differing definitions and the use of either continuous or categorical variables, two studies found that negative organizational climates were directly related to higher turnover (Glisson, Schoenwald, et al., 2008; Sheidow et al., 2007), while the other noted that a negative organizational climate was indirectly related to turnover, mediated by negative work attitudes (Aarons \& Sawitzky, 2006).

It is important to point out that some of the constructs used by Sheidow and colleagues (2007) as indicators of organizational climate have been studied as discrete variables in other studies. Specifically, one empirical study and one meta-analysis identified fairness, growth and promotional opportunities, role overload/stress, work group cooperation, and job satisfaction as 
unique predictors of turnover in different samples of general employees (Allen et al., 2003; Griffeth, Hom, \& Gaertner, 2000).

Demographic predictors of general workforce turnover. As with research specific to behavioral health staff, only two demographic variables were found to be associated with general workforce turnover. Specifically, a meta-analysis of turnover literature conducted in the 1990s indicated that an employee's number of children was positively associated with turnover, while the employee's tenure within the organization was negatively associated with turnover (Griffeth et al., 2000). It is interesting to note that these two demographic variables have not been investigated or reported on in most of the research specific to behavioral health staff turnover.

Organizational predictors of general workforce turnover. A number of organizational factors, in addition to those previously described, have been found to be significantly associated with general workforce turnover. For instance, a meta-analysis indicated that strong organizational commitment, positive leadership, and perceived autonomy were related to lower rates of turnover (Griffeth et al., 2000). A much older meta-analysis indicated that, above and beyond job satisfaction and organizational commitment, turnover intent was most predictive of actual turnover (Steel \& Ovalle, 1984). A follow-up study using both meta-analytic and path modeling procedures indicated that turnover intent mediated nearly every attitudinal contributor to turnover, again including job satisfaction and organizational commitment (Tett \& Meyer, 1993). These studies paved the way for more recent research to utilize turnover intent as a proxy for actual turnover. Unfortunately, this phenomenon has dominated the literature on predictors of turnover specific to the behavioral health field (e.g., Blankertz \& Robinson, 1997; Cropanzano et al., 2003; Lambert et al., 2001). Interestingly, these studies identified predictors that were nearly identical to the literature examining actual turnover. Two significant predictors of turnover intent 
that have not been studied in the literature on actual turnover were the availability of employment alternatives and job level (Mor Barak et al., 2001).

\section{Conceptual Model of Turnover}

Lambert and colleagues (2001) proposed a general model of the turnover process that neatly conceptualizes the complex associations between different types of predictors (Figure 2). Although this model is not specific to the behavioral health workforce, it encompasses the same broad constructs relevant to behavioral health staff. Specifically, Lambert and colleagues (2001) proposed that both demographic factors and organizational factors, in combination with alternative employment opportunities, are predictive of turnover intent. While the relation between organizational factors and turnover intent is mediated by work attitudes, demographic factors impact turnover intent both directly and indirectly. Finally, turnover intent is directly related to actual turnover. This conceptual model was based, in part, on a meta-analytic review of the general turnover literature in which a number of both demographic and organizational predictors were noted, with stronger effect sizes for the organizational predictors (Griffeth et al., 2000).

A logical inference to be made from the complex literature is that predictors of turnover vary across organizations, and also likely as a function of the population served by that organization (e.g., Blankertz \& Robinson, 1997). This study will examine the predictors of turnover in community behavioral health agencies involved in a state-wide initiative to implement Parent-Child Interaction Therapy (PCIT; Eyberg \& Funderburk, 2011; McNeil \& Hembree-Kigin, 2010). 


\section{Parent Study}

The current study uses data from a larger state-wide implementation initiative (NIMH R01 MH095750; A Statewide Trial to Compare Three Training Models for Implementing an $E B T$; PI: Herschell) in which the primary aim was to examine the relative effectiveness of different training models in promoting clinician use of PCIT. PCIT is a manualized parent training intervention with empirical support for use with families of children aged 2.5 to 7 years with disruptive behavior, or families with a history of physical abuse (McNeil \& Hembree-Kigin, 2010). Given the large evidence-base for the efficacy and effectiveness of PCIT (Thomas \& Zimmer-Gembeck, 2007), focus has now been placed on understanding the most effective way to implement PCIT in community treatment settings. As an initial step in this line of inquiry, the parent study is evaluating the effectiveness of three training methods common in the behavioral health field: (a) cascading model (CM); (b) learning collaborative (LC); and (c) distance education (DE; a full description of each training method is presented below).

The parent study presents a unique framework in which to study turnover for a number of reasons. First, some have speculated that rates of staff turnover would be greater in organizations implementing EBTs compared to those using treatment as usual (TAU) given the high degree of manualization and fidelity monitoring associated with most EBTs, as well as the subsequent lack of perceived autonomy reported by some clinicians implementing EBTs (Bjorklund et al., 2009; Novins, Green, Legha, \& Aarons, 2013). However, the few studies that have examined this hypothesis to date have found that EBTs may have a protective effect against turnover (e.g., Aarons, Sommerfeld, et al., 2009). Additional research is needed to more fully understand the impact that EBT implementation has on staff turnover. 
Second, the different training methods being assessed in the parent study vary substantially in terms of cost, time-intensiveness for the trainee, and quality control methods (see below for details regarding each training method). Given the inherent differences across these training methods, it is possible that rates of turnover could vary as a function of the type of training received by the participant. To our knowledge, this will be the first study investigating the possible effects of different training methods on staff turnover.

Third, characteristics of the specific EBT used in this study may impact turnover. The combination of challenging families and high rates of attrition seen in PCIT (e.g., Lanier et al., 2011; Lyon \& Budd, 2010) could contribute to higher levels of emotional exhaustion and depersonalization in clinicians (i.e., components of burnout; Maslach \& Jackson, 1981), both of which have been associated with greater turnover (Aarons \& Sawitzky, 2006; Sheidow et al., 2007). Although the parent study was not designed specifically to study turnover, care was taken to incorporate measures that would facilitate a prospective understanding of turnover. Each of these complexities within the parent study has created a unique context in which to study turnover specific to the field of behavioral health.

\section{Training Conditions}

Cascading Model. The cascading model (CM), also known as "train-the-trainer," is the training model that has been endorsed by the National PCIT Training Committee (Eyberg et al., 2009; Scudder \& Herschell, 2015). The initial training consists of 40 hours of direct contact with a PCIT trainer, followed by 16 hours of live training using real cases for 6 months. Participants also have bi-weekly contact with a trainer throughout the entire 12-month training phase. Following the 12-month initial training, clinicians participate in an additional 6 months of consultation and training (Herschell et al., 2015), at which point they are eligible to train others 
within their agencies. Clinicians involved in this first wave of training are referred to as "first generation clinicians;" the second group of clinicians that they train is referred to as "second generation clinicians."

Cascading model trainings require substantial up-front investment, with considerable time required to attend the initial training as well as the ongoing consultation. In addition, cascading model trainings for PCIT are expensive, with current rates at $\$ 4500$ per trainee (PCIT International Inc., n.d.). However, the primary benefit of a cascading model is that it allows trained clinicians (i.e., first generation) to return to their agencies and function as the trainer for other clinicians (i.e., second generation), with the intention of promoting more successful sustainability of the intervention without any effort needed by higher-ranking staff members (e.g., supervisors or administrators). Although research indicates that workshop trainings with ongoing follow-up like cascading models are effective in promoting clinician behavior change (Herschell, Kolko, Baumann, \& Davis, 2010), other research has suggested that there is a drift in fidelity to the intervention that occurs when the first generation of clinicians trains the second generation (Shore, Iwata, Vollmer, Lerman, \& Zarcone, 1995).

Learning Collaborative. The learning collaborative (LC) model takes a clinic-based approach to EBT implementation, and involves specialized training sessions for employees at each level of the organizational hierarchy (i.e., clinician, supervisor, and administrator). Within the current study, two clinicians, one supervisor, and one administrator from each clinic participated in the learning collaborative. Based on recommendations from the National Child Traumatic Stress Network, which routinely implements learning collaborative trainings (Markiewicz, Ebert, Ling, Amaya-Jackson, \& Kisiel, 2006), the learning collaborative condition for the parent study included three phases: pre-work, learning sessions, and action periods. The 
3-month pre-work phase consisted of a review of readings and materials, and conference calls with PCIT experts. Learning sessions were carried out over a 9-month period and consisted of three, two-day face-to-face meetings. Action periods occurred between learning sessions and incorporated the use of improvement data, technology, team meetings, and conference calls to support learning. In order to promote the sustainability of the intervention, clinics selected one supervisor and one clinician (i.e., first generation staff) who had completed the year-long intensive training to undergo additional training in order to enable them to train others (i.e., second generation staff) within their clinic (Herschell et al., 2015).

The learning collaborative model is perhaps the most time-intensive and costly of the three models. In fact, one recent study reported a cost of $\$ 11,523$ per clinician in a learning collaborative training (Dopp, Hanson, Saunders, Dismuke, \& Moreland, 2017). Despite the significant investment required, the intent of learning collaborative approach is to provide the entire clinic with the support and resources needed to promote the long-term sustainability of the intervention. However, there is mixed evidence regarding its ability to promote clinician behavior change and use of the intervention (reviewed in Herschell et al., 2015).

Distance Education. Distance education (DE) generally refers to a training model in which trainees learn the material at their own pace away from a traditional, face-to-face training setting. An online training course developed by the PCIT Team at the University of California, Davis (SAMHSA grant; PI: Urquiza) was used for the distance education condition within the current study. The training course included 11 modules and incorporated written materials, vignettes, videos, and quizzes; the entire training took clinicians approximately 10 hours to complete (Herschell et al., 2015). Consistent with the other two training models, each clinician in this condition was provided with the PCIT manual, the Dyadic Parent-Child Interaction Coding 
System (DPICS) Manual, the DPICS workbook, and phone consultation with a trainer (Herschell et al., 2015).

Advantages to the distance education condition include that it is free for trainees, and is the least time-intensive of the three training conditions. However, a review of training models for psychosocial interventions indicated that there are very few empirical studies examining selfdirected training and online training methods (Herschell et al., 2010). Additionally, the few studies that have examined self-directed and online trainings have shown that they generally do not produce positive outcomes in terms of clinician behavior or use of the intervention (Herschell et al., 2010; Jackson, Brabson, Quetsch, \& Herschell, under review). Thus, while the distance education condition might be appealing to clinicians as a result of its ease and lack of additional burdens, it is unclear whether it will produce the desired increase in clinician knowledge and skills.

\section{Purpose of the Current Study}

The current study explored demographic and organizational factors that predicted turnover in community behavioral health agencies involved in the state-wide initiative to implement PCIT. Turnover within the current study was defined as an employee separating from the original agency. Employees who changed roles and/or positions within the same agency were not included in turnover rates. Additionally, the current study differentiated between voluntary and involuntary turnover, as the lack of this distinction in previous turnover research has been a limitation. Finally, as there have been inconsistencies in the time frames used within the turnover literature, the current study included two different turnover rates: (a) across the 12-month training period; and (b) across the entire 24-month duration of data collection in the parent study. 
Although some studies have reported on similar PCIT implementation efforts, they only mentioned clinician turnover in passing and did not report on rates or predictors specific to their samples (e.g., Christian, Niec, Acevedo-Polakovich, \& Kassab, 2014; Pearl et al., 2012). Thus, the purpose of the current study was to explore turnover in a novel sample (i.e., PCIT clinicians) and a novel setting (i.e., community-based outpatient clinics implementing an EBT).

In the process of examining turnover in this new setting, the current study attempted to address some of the limitations found in previous turnover research. First, the current study used a clear definition of turnover, only including participants who left their agency (rather than those who changed roles within their agencies). Second, the outcome of interest in the current study was actual turnover, which differs from prior studies that have measured turnover intent. Third, the current study examined turnover for staff at different levels of the organizational hierarchy (i.e., clinicians, supervisors, and administrators), where prior research has focused almost exclusively on direct service providers. Fourth, the current study is unique in that the data regarding predictors of turnover were collected at multiple time points, thus allowing a prospective, rather than retrospective (which is most common in the turnover literature) understanding of what factors contributed to turnover. Finally, the current study examined the association between turnover and client outcomes, which has been hypothesized to exist but has been the focus of only a few studies with inconsistent findings (Garner et al., 2013; StrolinGoltzman et al., 2010; Williams \& Potts, 2010). 


\section{Hypotheses}

\section{Aim 1}

The first aim of the current study was to explore rates of turnover for staff members involved in the larger implementation initiative. Are there different turnover rates for clinicians, supervisors, and administrators? Are staff turnover rates influenced by training model?

It was hypothesized that there would be different turnover rates for clinicians, supervisors, and administrators. Research has indicated that a higher job level within an organization is associated with lower turnover intent (Mor Barak et al., 2001). Given the strong association between turnover intent and actual turnover in general workforce studies (Steel \& Ovalle, 1984; Tett \& Meyer, 1993), it was hypothesized that lower-ranking employees (i.e., clinicians) would have higher rates of turnover compared to higher-ranking employees (i.e., supervisors and administrators).

Given that research has found that strong organizational support can protect against turnover (Allen et al., 2003), it was hypothesized that rates of clinician turnover would be lower in the learning collaborative condition, in which staff members at all levels of the organizational hierarchy are involved in the implementation initiative. To date, research investigating factors that influence turnover within implementation initiatives has focused exclusively on clinicians; thus no hypotheses were made regarding the effect of training condition on supervisor and administrator turnover.

\section{$\operatorname{Aim} 2$}

The second aim of the current study was to identify predictors of turnover for clinicians, supervisors, and administrators involved in the parent study. Based on the literature, it was hypothesized that both demographic (Ben-Dror, 1994b; Griffeth et al., 2000; Sheidow et al., 
2007) and organizational factors (Aarons \& Sawitzky, 2006; Glisson et al., 2008; Sheidow et al., 2007) would predict turnover. It was also hypothesized that the organizational factors would be more predictive of turnover than demographic factors (Griffeth et al., 2000).

\section{$\operatorname{Aim} 3$}

The final aim of the proposed study was to explore the hypothesized relation between clinician turnover and therapeutic outcomes. Due to the study design and inconsistencies across prior studies (Garner et al., 2013; Strolin-Goltzman et al., 2010; Williams \& Potts, 2010), this aim was exploratory in nature.

\section{Method}

\section{Participants}

Data collected from clinicians, supervisors, administrators, and families participating in the state-wide implementation initiative (NIMH R01 MH095750; A Statewide Trial to Compare Three Training Models for Implementing an EBT; PI: Herschell) was used for the current study. Participants included 100 clinicians, 50 supervisors, 50 administrators who were recruited to participate in the larger study described above. In addition, 110 families who were enrolled by first generation clinicians (i.e., those who were trained in the first group of the cascading model and learning collaborative) and had completed the Eyberg Child Behavior Inventory (ECBI; described below) at baseline were included in the current sample.

Outpatient clinics and their staff members were recruited by research study team members. Clinic inclusion criteria were: (a) psychiatric outpatient clinic licensure in Pennsylvania, (b) willingness to participate in PCIT training, (c) the ability to cover site preparation costs, and (d) agreeable to research participation (Herschell et al., 2015). Clinic administrators were defined as an Executive Director, Chief Financial Officer, or other 
individual responsible for daily operations at an enrolled clinic; there were no other inclusion criteria for administrators (Herschell et al., 2015). Supervisors were eligible to participate if they were employed at an enrolled agency, had been identified by the administrator as the program lead, and were willing to participate in training if they were assigned to the learning collaborative condition (Herschell et al., 2015). Clinician inclusion criteria were as follows: (a) employment at an agency that had elected to participate in PCIT training, (b) masters or doctoral degree in a human services field, current licensure in his/her field or receiving supervision from a licensed individual, (c) a current caseload that included clients appropriate for PCIT, d) receptive to receiving PCIT training and (e) have not been previously trained, and willing to complete research-related tasks (Herschell et al., 2015).

All families enrolled in PCIT services with a participating clinician were eligible, unless the child was a ward of the state or living under state custody (Herschell et al., 2015). Clinicians recruited families by presenting all eligible families on their caseloads with a "permission to contact" form. If caregivers were interested in participating in the study, clinicians instructed them to provide their contact information and sign the "permission to contact" form, which was then sent to the study team. Interested caregivers were then contacted by a staff member from the research team who obtained informed consent. Only families who had been enrolled by a first generation clinician were eligible for inclusion in the current study, as second generation clinician data collection had not been complete at the time of analyses.

\section{Procedures}

Participating clinicians, supervisors, and administrators completed a battery of assessments at four time points: baseline, 6- (mid), 12- (post), and 24-months (1 year follow-up). If a member of the research team learned of a staff member leaving the agency at any point 
outside of the assessment windows, they followed up with the staff member in order to have them complete the Agency Staff Change form (described below). This was to ensure accurate reporting of turnover rates and to measure variables associated with turnover as close to the time of turnover as possible. Additionally, participating caregivers completed an assessment battery at four time points (baseline, 3-, 6-, and 12-months). The battery contained a variety of questionnaires including the Demographic Information Form and the Eyberg Child Behavior Inventory (ECBI), which are the only caregiver-child measures of relevance to the current study. In order to maximize the amount of data collected, participants were provided with a number of methods to complete their questionnaire batteries. The majority of participants (86\% of clinicians and supervisors, and $61 \%$ of families) chose to complete their questionnaires online. If participants did not have internet access, they were given the option of completing the questionnaires over the phone or completing paper copies.

\section{Measures}

Please see the Appendix for all measures used in the current study.

Demographics. Demographic information was collected using the Administrator Background Form (completed by administrators), the Supervisor Background and Contact Information Form (completed by supervisors), the Clinician Background and Contact Form (completed by clinicians), and the Demographic Information Form (completed by families). Each of these forms asked for standard demographic information (e.g., gender, race, education level). Additionally, the forms for agency staff members asked about information regarding the respondent's current role, such as the amount of time employed by the agency and the amount of experience within the human services industry. Demographic information was only collected during the baseline assessment. 
Agency Staff Change Form. The Agency Staff Change Form contained questions related to changes in employment. Because it is possible for staff to move between two clinics enrolled in the parent study, these forms were administered to clinicians, supervisors, and administrators at all four time points. As previously mentioned, study team members also completed the form if they learned about a staff member leaving the original agency at any point during the study outside of assessment windows. Both the Agency Staff Change Form and the Demographics form have been used in previous implementation trials (K23 MH074716; PI: Herschell; Kolko et al., 2012)

Organizational Readiness for Change (ORC). The Organizational Readiness for Change questionnaire (ORC; Lehman, Greener, \& Simpson, 2002) is a 115-item self-report questionnaire completed by supervisors and administrators during the baseline, 12-month, and 24-month assessment periods. This questionnaire was designed as a comprehensive assessment of an organization's overall functioning and readiness for change. When completing the ORC, participants rated their level of agreement with each item on a 5-point Likert-type scale (1disagree strongly, 2-disagree, 3-uncertain, 4-agree, 5-agree strongly). The ORC includes four main scales (italicized), each with a number of subscales (listed in parentheses): (a) motivational factors (program needs, training needs, pressures for change); (b) program resources (office facilities, staffing, training, equipment, internet); (c) staff attributes (growth, efficacy, influence, adaptability, satisfaction); (d) organizational climate (mission, cohesion, autonomy, communication, stress, change, leadership).

Of particular relevance to the current study are the organizational climate and program resources scales. The items on these scales map onto the constructs of organizational climate and culture (respectively) that have been found to be predictive of staff turnover. As such, the 
organizational climate scale was used as an indicator of organizational climate and the program resources scale was used as an indicator of organizational culture in the current study. Scores on these scale range from 10 to 50, with higher scores indicating more positive ratings of the climate or culture.

Research has indicated that responses on the ORC have adequate psychometric properties. Cronbach's alpha was computed for each of the subscales as an estimate of internal consistency, and was adequate (above .70) for the majority of the subscales (Lehman et al., 2002). Responses from the current sample of supervisors and administrators indicated adequate internal consistency for the organizational climate $(\alpha=0.81)$ and program resources $(\alpha=0.68)$ scales.

Survey of Organizational Functioning (SOF). The Survey of Organizational Functioning (SOF) is a 162-item self-report questionnaire that was developed based on the ORC and was completed by clinicians in the current study at baseline, 12-month, and 24-month assessment periods. Participants rated their level of agreement with each item on a 5-point Likert-type scale. Because the SOF is geared toward clinicians, there are more items and scales than on the ORC. The SOF includes seven main scales (italicized) and a number of subscales (listed in parentheses): (a) change (program needs, training needs, pressures for change); (b) resources (offices, staffing, training, computer access, e-communications); (c) staff attributes (growth, efficacy, influence, adaptability); (d) organizational climate (mission, cohesion, autonomy, communication, stress, change); (e) job attitudes (burnout, satisfaction, director leadership); (f) workplace practices (peer collaboration, deprivatized practice, collective responsibility, focus on outcomes, reflective dialogue, counselor socialization); (g) training 
exposure and utilization (training satisfaction, training exposure, training utilization-individual level, training utilization-program level).

As with the sample of supervisors and administrators, the organizational climate and resources scales will be used as indicators of organizational climate and organizational culture, respectively. Scores on these scale range from 10 to 50, with higher scores indicating more positive ratings of the climate or culture. Although no published reports on the psychometrics of the SOF currently exist, results from the current sample of clinicians indicated acceptable internal consistency for both the organizational climate $(\alpha=0.83)$ and the resources $(\alpha=0.68)$ scales.

Eyberg Child Behavior Inventory (ECBI). Therapeutic outcomes were assessed using the Eyberg Child Behavior Inventory (ECBI; Eyberg \& Pincus, 1999). The ECBI is a 36-item caregiver-report measure designed to assess externalizing behavior problems in children between 2 and 16 years of age, and is commonly used in PCIT to measure progress throughout treatment. In the current study, caregivers completed the ECBI at baseline, 3-, 6-, and 12-month assessment periods. Caregivers rated each item on two scales: a 7-point Likert-type scale to measure the intensity of the behavior (i.e., Intensity Scale), and a dichotomous yes/no scale indicating whether or not they believe the behavior is a problem (i.e., Problem Scale). Higher scores on each of these scales indicate greater child behavior problems, with a score of 131 on the Intensity Scale and/or a score of 15 on the Problem Scale indicating clinically-significant behavior problems (Rich \& Eyberg, 2001).

Responses on the Intensity Scale have demonstrated strong test-retest and inter-rater reliabilities ( $r=.75$ and .86, respectively; Eyberg \& Pincus, 1999). Responses on the Problems Scale also demonstrated strong inter-rater reliability ( $r=.79$; Eyberg \& Pincus, 1999). Boggs, 
Eyberg, and Reynolds (1990) found that responses on the Intensity Scale demonstrated evidence of concurrent validity with the Internalizing and Externalizing subscales on the Child Behavior Checklist (CBCL; Achenbach, Dumenci, \& Rescorla, 2002; $r=.75, r=.67$, respectively).

\section{Definition of Turnover}

Turnover as assessed within the current study was defined as an employee separating from the original agency. Employees who changed roles and/or positions within the same agency were not included in the turnover count. Additionally, the current study differentiated between voluntary and involuntary turnover, and only included participants who voluntarily left their agencies. Finally, turnover rates were calculated for both the 12-month training period and for the entire 24-month study duration.

\section{Data Analyses}

All analyses were conducted either in Statistical Package for the Social Sciences (SPSS version 24.0, IBM Corp, 2016) or in HLM, version 7 (Raudenbush, Bryk, \& Congdon, 2011). For Aim 1, 12-month and 24-month rates of turnover were calculated for clinicians, supervisors, and administrators. A series of chi-square tests of independence were used to determine if turnover rates differed by job type or by training condition.

For Aim 2, supervisors and administrators were combined to form one sample. This decision was made given their higher positions within the organization and different day-to-day responsibilities compared with clinicians, their shared measure of organizational climate and culture (i.e., the ORC), their similar roles within each training condition, and in order to maximize power. Two logistic regressions were used to test for significant demographic predictors of (a) clinician turnover, and (b) supervisor/administrator turnover. 
A different analytic plan was required for the organizational predictor variables, given the nested structure of the data (staff members within agencies). This was deemed unimportant for demographic variables, as they are not subject to change due to the influence of agency-level factors. However, organizational variables are conceptually related to agency-level factors, and thus standard logistic regression models would not be appropriate due to the violation of the assumption of independence. As such, Hierarchical Linear Modeling (HLM) was used in order to account for the nested structure of the data in assessing organizational predictors of turnover. A series of HLM analyses were run to assess for significant organizational predictors of (a) clinician turnover, and (b) supervisor/administrator turnover.

For Aim 3, a more descriptive approach was taken. Although 110 families were originally enrolled, a number of issues prevented the use of inferential statistics. Specifically, high rates of family attrition were noted, and only 26 families were assigned to clinicians who left during the study, thus limiting the sample size. As such, the current study takes an in-depth look at the outcomes for individual families whose clinicians left at some point during their treatment phase.

\section{Results}

\section{Sample Characteristics}

Table 1 contains the full demographic information for clinicians, administrators, and supervisors. Clinicians $(n=100)$ were primarily Caucasian $(91 \%)$ and female $(84 \%)$, and were an average of 39 years old $(S D=10.04)$ at baseline. The majority $(92 \%)$ held a Master's degree in either in psychology (37\%) or social work (31\%), and half (52\%) were professionally licensed. Most clinicians were employed full time (74\%) and reported an average yearly salary of $\$ 43,939(S D=\$ 12,712)$, while those who were employed part time (28\%) reported an average 
hourly wage of $\$ 28.55(S D=\$ 6.77)$. Clinicians had an average of 11.42 years $(S D=8.20)$ experience within the human service industry and an average of 4.82 years $(S D=5.61)$ at their current agencies.

Supervisors $(n=50)$ were also primarily Caucasian $(88 \%)$ and female $(80 \%)$ and were an average of 45 years old $(S D=9.48)$ at baseline. Similar to the clinicians, the majority of supervisors held a Master's degree (82\%), while the remainder held a Doctoral degree. Most held their degrees in either psychology (32\%) or social work (36\%). Most supervisors (86\%) were employed full-time and reported an average yearly salary of $\$ 55,991(S D=\$ 11,132)$, while those who were employed part-time $(n=5,<1 \%)$ reported an average hourly wage of $\$ 29.40$ $(S D=\$ 4.93)$. Supervisors had an average of 18.26 years $(S D=8.60)$ experience within the human service industry and an average of 7.20 years $(S D=5.45)$ years within their current agencies.

As with clinicians and supervisors, administrators $(n=50)$ were primarily Caucasian $(90 \%)$ and female $(62 \%)$, and were an average of 48 years old $(S D=8.96)$ years old at baseline. The majority of administrators held a Master's degree (68\%) or a Doctoral degree (20\%). Most administrators had a degree in social work (32\%), with a substantial number holding degrees in psychology (22\%) or another field (30\%). Administrators in the current sample reported an average of 22 years $(S D=8.53)$ experience in the human service industry and had worked an average of 12 years $(S D=8.40)$ at their current agencies.

Table 2 contains demographic information for families. Children in the current sample were primarily Caucasian $(68 \%)$ and male $(61 \%)$, with an average age of 5.26 years $(S D=1.63)$ at the time of enrollment. Caregivers were primarily Caucasian (68\%) and female (96\%), were typically the child's biological mother (90\%), and were an average of 32.29 years old $(S D=$ 
8.18) at the time of enrollment. Most were high school graduates (32\%) and had an annual family income of $\$ 14,999$ or less $(38 \%)$.

\section{Aim 1: Rates of Turnover}

In order to maintain a clear picture of turnover, two different rates of turnover were calculated: one from the time of participant enrollment to the 12-month assessment, and one from the time of participant enrollment through the entire 24-month duration of the study. Within the 12 months, $11 \%$ of clinicians, $6 \%$ of supervisors, and $4 \%$ of administrators left their respective agencies. By the end of the 24-month study duration, $31 \%$ of clinicians, $30 \%$ of supervisors, and $26 \%$ of administrators had left their respective agencies (Table 3 ).

Two chi-square tests of independence were run to test the hypothesis that staff in higherranking roles (i.e., supervisors and administrators) would have lower rates of turnover than clinicians. Contrary to hypotheses, results of the chi-square test indicated that there were no differences in the percentage of clinicians, supervisors, or administrators who left during the first year of the study $\left(\chi^{2}[2, n=196]=0.24, n s\right)$ or over the 24-month course of the study, $\left(\chi^{2}[2, n=\right.$ 191] $=0.46, n s ;$ see Table 3).

A second series of chi-square tests of independence were run to determine if there were different rates of clinician, supervisor, and administrator turnover based on training condition. Contrary to hypotheses, results indicated that there were no differences in the rates of turnover for clinicians in each training condition at the 12-month time point $\left(\chi^{2}[2, n=96]=2.10, n s\right)$ or by the end of the study $\left(\chi^{2}[2, n=95]=0.51, n s\right)$. Additionally, there were no significant differences in the 12-month rates of turnover for supervisors $\left(\chi^{2}[2, n=50]=2.02, n s\right)$ or administrators $\left(\chi^{2}[2, n=50]=0.96, n s\right)$. However, differences in 24-month turnover rates based on training condition for supervisors $\left(\chi^{2}[2, n=48]=0.51, p=.08\right)$ and administrators $\left(\chi^{2}[2, n=\right.$ 
$48]=4.75, p=.09)$ approached significance. For both supervisors and administrators, rates of turnover were greater in the learning collaborative condition than in the cascading model or distance education conditions (Table 4). Figures 3-5 visually depict the increase in turnover rates from 12-months to 24-months for clinicians, supervisors, and administrators in each of the three conditions.

\section{Aim 2: Predictors of Turnover}

Variable selection. A thorough review of the literature was conducted to identify possible predictors of turnover relevant to the current study. Because there is limited research pertaining specifically to behavioral health staff turnover, predictor variables within the general workforce literature were also considered for the current study. Although a large number of variables were identified as potentially relevant, (refer to Figure 1), a limited number of these variables were selected for inclusion in the current study in order to maximize power.

Between both the general workforce literature and the behavioral health workforce literature, six demographic predictors of turnover were identified (i.e., age, education, salary, tenure with the agency, number of children, and alternative opportunities). Four of these variables (i.e., age, education, salary, and tenure with the agency) were selected for inclusion in the current analyses, given that they were assessed in the larger parent study. However, data on annual salary was not collected for administrators; thus, salary was not included as a predictor for the supervisor/administrator group. Finally, based on results of these preliminary analyses, education was excluded from regression analyses for clinicians due to lack of variability (92\% of clinicians held a Master's degree and 8\% held a Doctoral degree).

Fifteen organizational variables were identified as significant predictors of turnover based on existing literature (refer to Figure 1). Of these variables, organizational culture and 
organizational climate were selected for inclusion in the current analyses. These two variables are multidimensional constructs that encapsulate many of the other unidimensional variables that were identified in the literature (e.g., job stress/burnout, work attitudes, organizational support). Thus, they were selected as the most parsimonious representations of the literature and were also already being measured in the parent study. Two other organizational variables - service structure and agency size - were excluded from analyses in order to maximize power, given that they were not as strongly supported in the literature.

Preliminary analyses. For demographic predictors, a series of preliminary analyses were used to check for outliers, violations of normality, homogeneity of variances, and multicollinearity. Skewness and kurtosis values were between -2 and +2 , which are considered acceptable limits for normality (Tabachnick \& Fidell, 2014), for all variables (both for the clinician group and the supervisor/administrator group) except for tenure with the agency. This variable had five outliers (as indicated by $z$-scores greater than 3.2); three clinicians had worked at their respective agencies for 26, 28, and 32 years, while two administrators had worked at their respective agencies for 33 and 35 years. Given that the purpose of this study is to understand factors that predict why people leave their agencies, it was decided that this variable would be transformed rather than deleting the outliers. Given that these participants had remained at their agencies for so long, they could contribute important information to the understanding of turnover. Thus, a logarithmic transformation was computed for the "tenure with agency" variable which resulted in acceptable skewness and kurtosis values. Although this transformed variable was included in regressions, the untransformed means and standard deviations are reported for ease of interpretation (Tabachnick \& Fidell, 2014). 
Correlations were also computed between all variables of interest to assess for multicollinearity (Table 5). As would be expected, age and tenure with the agency were significantly correlated, but not to the extent that would indicate multicollinearity. For supervisors and administrators, the two organizational variables of interest were also significantly correlated, but not to the extent that would indicate multicollinearity. For clinicians, however, the correlation between the two organizational variables was slightly greater than 0.70 , which it typically the cut-off for multicollinearity (Tabachnick \& Fidell, 2014). However, it was not much greater than this cut-off $(r=0.73)$, and there is substantial literature to support the inclusion of these two variables as distinct but related constructs (Glisson, Landsverk, et al., 2008). As such, organizational climate and culture were included in analyses for both the clinician group and the supervisor/administrator group.

Before HLM analyses were computed, an unconditional model was run to test for the amount of variance in staff turnover that could be accounted for by nesting. This was achieved through a one-way analysis of variance in which the outcome variable (turnover) was entered without any additional predictors. This test provides an intraclass correlation coefficient (ICC), which is an estimate of the variance in level-one factors that can be accounted for by level-two factors. Two unconditioned models were run, one to understand the effect of nesting on clinician turnover, and one to understand the effect of nesting on supervisor and administrator turnover. Results indicated that about $18 \%$ of the variance in clinician turnover was accounted for at the agency level, while $79 \%$ of the variance in supervisor and administrator turnover was accounted for at the agency level. Both of the ICCs indicate that a significant amount of variance in turnover is accounted for by agency-level factors and supported the use of HLM to account for this shared variance. 
Demographic predictors. A simultaneous logistic regression model was computed in order to predict clinician turnover based on age, salary, and tenure with the agency. A test of the full model was not significant, $\chi^{2}(3)=0.72, n s$. For this sample of clinicians, age, salary, and tenure with the agency were not significant predictors of turnover. A second simultaneous logistic regression model was computed to predict supervisor and administrator turnover based on age, and tenure with the agency. Again, the test of the full model was not significant, $\chi^{2}(2)=$ $2.86, n s$. For this sample of supervisors and administrators, age and tenure with the agency did not significantly predict turnover (Table 6).

Organizational predictors. Separate files were created in SPSS for the each level of the data and for both the clinician group and the supervisor/administrator group. The level-one data files included each participant's scores on the measures of organizational culture and climate. The level-two data files included dummy codes for training condition, as agencies were randomized to training conditions and it was hypothesized that differences in training conditions could influence participants' perceptions of their workplace. Once data files had been created and cleaned, they were imported into the HLM software (Raudenbush et al., 2011) for analyses. Each model was run twice, once without training condition included and once with training condition included.

Results indicated that perceptions of organizational culture did not predict clinician turnover, either before (Coefficient $=-0.05, S E=0.08, t=-0.17, d f=47, p=0.53$ ) or after (Coefficient $=-0.05, S E=0.08, t=-0.63, d f=47, p=0.53)$ training condition was added to the model. Organizational climate was also not a significant predictor of clinician turnover either before $($ Coefficient $=-0.02, S E=0.07, t=-0.35, d f=47, p=0.72)$ or after $($ Coefficient $=-0.03$, $S E=0.07, t=-0.37, d f=47, p=0.72)$ taking training condition into account. Organizational 
culture was also not a significant predictor of supervisor and administrator turnover before $($ Coefficient $=0.03, S E=0.08, t=0.36, d f=47, p=0.73)$ or after $($ Coefficient $=0.03, S E=0.08$, $t=-0.36, d f=45, p=0.72)$ accounting for training condition. However, organizational climate did significantly predict supervisor and administrator turnover both without training condition $($ Coefficient $=-0.14, S E=0.07, t=-2.09, d f=47, p=0.04)$ and with training condition accounted for (Coefficient $=-0.16, S E=0.07, t=-2.20, d f=47, p=0.03$ ). See table 6 for all HLM statistics. These results indicated that supervisors and administrators with greater organizational climate scores (i.e., more positive perceptions of the workplace) were less likely to leave their agencies.

\section{Aim 3: Therapeutic Outcomes}

To examine the association between clinician turnover and client outcomes, families were first categorized based on the likelihood that they had experienced service disruption as a result of clinician turnover. For the purposes of this analysis, service disruption was defined in terms of when the clinician left in relation to the typical length of PCIT treatment. Once families were identified as having experienced likely service disruption, their outcomes over time as measured on the ECBI were examined.

Figure 6 shows the process by which families were identified as possibly having experienced service disruption as a result of clinician turnover. Of the 110 families originally enrolled in the study, 24\% $(n=26)$ had been assigned to a clinician who left at some point during the duration of the study. These 26 cases were further analyzed using a descriptive approach to better understand the degree to which clinician departure may have impacted their outcomes. Several criteria were examined for this more in-depth analysis: (a) how far along in treatment families were when their clinicians left; (b) how this treatment duration matched with typical 
PCIT treatment length; (c) whether or not they had been assigned to a new clinician within the implementation trial; (d) how many time points for which they had completed measures; and (e) the change in their ECBI intensity scores from the first time point to the last available time point. Based on these criteria, families were categorized as one of the following: (a) unlikely service disruption ( $n=11,42.3 \%$ ): (b) possible service disruption $(n=10,38.5 \%)$; or (c) likely service disruption $(n=5,19.2 \%)$.

Unlikely service disruption. Typical PCIT treatment lasts for about 12 to 16 weekly sessions, for a total duration of three to four months (assuming regular attendance and few missed sessions). Based on this criterion, families $(n=10)$ whose clinician left more than four months after they had been enrolled in the study were considered unlikely to have experienced disrupted service. Six families had enrollment dates that were after the departure date of their assigned clinician. This was likely due to a procedural lag between when clinicians informed families about the study and when research staff was able to contact the family to enroll them. The clinician assigned to one of these families moved to a different agency within the study, and transferred that family to the new agency. Thus, this family was categorized as unlikely to have experienced service disruption, resulting in a total of 11 families in this category

Possible service disruption. One family whose enrollment date was later than their clinician's departure date withdrew from the study. The cause for their withdrawal is unknown and no other information was available; as such, they were categorized as possibly having experienced service disruption. Very little information was available for the remaining four families whose enrollment date was later than their clinician's departure date, although they were all assigned to the same clinician. There is no indication that they were transferred to a different clinician within their original agency. However, all four of them failed to complete assessments 
following the baseline time point, which could be indicative of premature termination from treatment and, as such, they were categorized as having experienced possible service disruption.

Ten families whose clinician departures occurred within four months of their enrollment dates were identified. None were transferred to new clinicians and none went with their clinicians to new agencies. Five failed to complete any measures after baseline, likely indicative of dropout, potentially related to their clinician leaving. However, in the absence of additional information, they were categorized as possibly having experienced service disruption, for a total of ten families in this category.

Likely service disruption. Five remaining families had clinicians whose departure dates occurred within four months of their enrollment. These five families were categorized as having experienced likely service disruption, given the temporal relation between when they were enrolled and when their clinicians departed. For these five families, baseline, 3-month, and 6month assessment data are available. As is standard practice in PCIT, ECBI scores from these time points were compared to determine if the child experienced any change in behavior problems during this time. Figures 7 and 8 depicts their change in ECBI Intensity and Problem scores, respectively, across the assessment time points in relation to the clinical cutoff score on the ECBI.

Family one, whose clinician left approximately two and a half weeks into treatment, experienced an 81 point decrease in ECBI Intensity scores and had an ECBI problem score of 0 at the 6 month assessment point. At baseline, this family was below the clinical cutoff score on the ECBI, and it is possible that one or two sessions were sufficient for the family to see positive changes in their child's behavior. Thus, although this family likely experienced service disruption as a result of their clinician leaving, it did not appear to impact their clinical outcomes. 
Family five reported a decrease of one point on the ECBI Intensity scale and an increase of eight points on the ECBI Problem scale, which is essentially equivalent to no change. Additionally, this family's scores remained stable within a few points of the clinical cutoff score. However, this family's clinician left 3.5 months after their enrollment, so it is unclear the extent to which the lack of change in behavior problems was related to clinician turnover. The remaining three families (families 2, 3, and 4) reported an increase in behavior problems from baseline to 6 months. Although increases in behavior problems are sometimes seen during the middle of treatment when discipline is introduced to the child, it is possible that these increases are related to clinician turnover. These same families also indicated increases in behavior problems from baseline to 3-months, and all of their clinicians left at 2-months. Although much more information would be needed to substantiate this hypothesis, it is worth considering that clinical outcomes for this small subset of families were indeed impacted by clinician turnover.

\section{Discussion}

\section{Rates of Turnover}

Overall, the current study experienced a 12-month turnover rate of $8 \%$ and a 24-month turnover rate of $30 \%$ for all behavioral health staff combined. Although no analyses were run to statistically compare rates of turnover in the current to those reported in the literature, this annual rate appears much lower than typically experienced in community treatment settings (30-50\%; Bjorklund et al., 2009; Bukach et al., 2015; Garner, Hunter, Modisette, Ihnes, \& Godley, 2012; Selden, 2010). Other researchers reporting on clinician turnover within EBT implementation initiatives have also reported lower rates (Aarons, Fettes, et al., 2009; Beidas et al., 2015; David J. Kolko et al., 2012) for clinicians using EBTs compared with those using TAU. These findings 
have caused researchers to hypothesize that the use of an EBT has a protective effect against clinician turnover, which may be consistent with the results of the current study.

However, the 24-month rate of turnover within the current study is more comparable to turnover rates reported across similar time spans in other EBT implementation initiatives (Aarons, Fettes, et al., 2009; Beidas et al., 2015; David J. Kolko et al., 2012). Although results did not indicate statistically-significant differences in the rates of turnover for different types of staff members, clinicians trended toward greater turnover rates than supervisors and administrators, particularly at the 12-month time point. Given the small proportion of individuals who left their agencies at the 12-month time point, it is possible that statistical differences would have been noted with a larger sample size. To the author's knowledge, this is one of the first studies to report separate rates of turnover for clinicians, supervisors, and administrators, although prior research has indicated that those with positions higher on the organizational hierarchy are less likely to leave their agencies (Mor Barak et al., 2001). It is important to understand differences between these distinct groups, given their different roles and day-to-day responsibilities.

This was also one of the first studies to investigate the effects of specific training conditions on turnover. Although previous studies have examined the effect of EBT implementation on clinician turnover (e.g., Aarons, Fettes, et al., 2009; Herschell, Kogan, Celedonia, Gavin, \& Stein, 2009; Kolko et al., 2012), to date no study has examined how different training conditions within EBT implementation may influence staff turnover. Contrary to hypotheses, clinician turnover did not differ based on training condition. One possible explanation for this null finding is that clinicians across all groups were highly motivated to complete training, given the high rate of referrals for children with disruptive behaviors (Lavigne 
et al., 1998) and the resultant industry value associated with PCIT certification. It was hypothesized that the learning collaborative condition would have lower rates of clinician turnover given the high level of organizational support presumed to underlie this training method (Cavaleri et al., 2006). However, clinician motivation to become certified in PCIT across all training conditions may have been strong enough to promote clinician retention through training completion, despite any possible differences in perceived organizational support.

This explanation is also consistent with very low 12-month rate of clinician turnover (11\%) compared with the higher 24-month rate of turnover (31\%). It is especially interesting to note that at the end of the 12-month period, clinicians were just finishing up with their initial training period. Although new opportunities for employment has been identified as a predictor of turnover (Griffeth et al., 2000), it was not assessed in the current study. It is possible that clinicians in the current study were participating in training in order to make themselves more competitive for new job opportunities, and left just after completing training once they were eligible for PCIT certification. In fact, this issue was reported in a previous study in which agency administrators were interviewed regarding barriers to EBT implementation (Herschell et al., 2009).

An additional possible explanation for the lack of different turnover rates across training conditions was that all training costs (e.g., cost of registration, materials, etc.) were covered and days that clinicians took off from work to attend trainings were reimbursed through the grant that funded the larger parent study (Herschell et al., 2015). Thus, clinicians did not experience any out-of-pocket expenses and did not have the burden of advocate to supervisors, other authority figures, or managed care companies to have their cost of training covered. As such, the training experience for clinicians in the study may not be analogous to that of typical community 
behavioral health providers. Perhaps different rates of turnover would have been noted across training conditions if clinicians had been exposed to some of the burdens that may accompany EBT training in typical community treatment settings.

Although there were no significant differences in rates of clinician turnover across training conditions, differences that approached statistical significance were noted in the rates of supervisor and administrator turnover and likely would have reached statistical significance with a larger sample. Specifically, by the end of the 24-month study duration, nearly half of the supervisors and administrators in the learning collaborative condition had left their agencies, whereas only $19 \%$ of supervisors and $13 \%$ of administrators had left in the cascading model condition, and $24 \%$ of both supervisors and administrators had left in the distance education condition. This is a particularly interesting finding given that the learning collaborative condition is the only training condition that actively involves the supervisors and administrators; the cascading model and distance education conditions only require clinician participation. Taken together, the null finding for clinicians and significant finding for supervisors and administrators suggest that the additional effort (above and beyond typical expectations for these particular job types) that accompanies training could influence turnover. Specifically, there is a relatively equivalent amount of work for clinicians in each training condition, which could explain why no single condition had higher rates of clinician turnover. However, expectations and responsibilities for supervisors and administrators are greater in the learning collaborative condition than the other two conditions. Thus, it is plausible that, although the learning collaborative condition is designed to promote long-term sustainability of the intervention through increased organizational support at all levels, the extra burden placed on those higher in the organizational hierarchy are aversive enough to drive them away. 


\section{Predictors of Turnover}

Results of the current study were inconsistent with prior research indicating that demographic variables (i.e., age, tenure with the agency, and salary) were predictive of turnover (Beidas et al., 2015; Ben-Dror, 1994a; Bukach et al., 2015; Griffeth et al., 2000). Within the current sample, no demographic variables were significant predictors of staff turnover for either clinicians or supervisors and administrators. Unfortunately, a number of potential variables of interest, such as education for both groups and salary for supervisors and administrators, were excluded from analyses due to measurement issues. It is possible that, given the complex nature of turnover, including these variables in the model may have resulted in better prediction of turnover for the current sample. However, one study reported that organizational factors were more predictive of turnover than demographic factors (Griffeth et al., 2000). This converges with findings for supervisors and administrators in the current sample, for whom organizational climate did significantly predict turnover. It is understandable that negative perceptions of the workplace (e.g., workplace cohesion, job autonomy, role stress, etc.) would be more influential in one's decision to leave a job than age or tenure with the agency.

It is interesting to note that organizational climate predicted turnover for supervisors and administrators, both with and without training condition taken into account. Although results of a one-way ANOVA assessing for differences in organizational climate based on training condition were not significant, supervisors and administrators in the learning collaborative condition had the lowest average rating of organization climate compared to supervisors and administrators in the other two conditions. Given that supervisors and administrators in the learning collaborative condition were more likely to leave than those in the other two conditions, it is possible that the added burdens associated with this training condition negatively influenced their perceptions of 
workplace climate, thus contributing to their decision to leave. However, it is interesting that organizational culture, which corresponds to the day-to-day workplace practices, was not predictive of turnover. As such, the findings within the current sample suggest that self-reported daily workplace practices and responsibilities did not differ for supervisors and administrators based on training condition, but their perceptions of and attitudes toward the workplace environment did. It is important to keep in mind that culture and climate are multidimensional constructs. Although a small sample size and limited power precluded a more fine-tuned analysis of the underlying unidimensional indicators of organizational climate, it would be interesting to know if there were particular facets of organizational climate that were drivers of this significant finding.

For supervisors and administrators in the current sample, findings were partially consistent with prior research indicating that negative organizational climate predicted turnover (Aarons \& Sawitzky, 2006; Glisson, Schoenwald, et al., 2008; Sheidow et al., 2007). However, findings in the current study were inconsistent in that organizational culture and climate were not significant predictors of clinician turnover. Unfortunately, no variables were identified as predictors of clinician turnover. Given that turnover is a complex process and variables had to be selected for inclusion in the current study in order to maximize analytic power, it is possible that some important variables were excluded from analyses.

\section{Client Outcomes}

Researchers have long hypothesized that clinician turnover negatively impacts client outcomes. However, this hypothesis has received mixed support in the few studies that have directly examined it (Garner et al., 2013; Strolin-Goltzman et al., 2010; Williams \& Potts, 2010). In the current study, a small sample size and high rates of family attrition precluded group-based 
analyses to compare outcomes for families whose clinician left versus families whose clinicians remained throughout the course of treatment. However, a more descriptive analysis indicated that $24 \%$ of the families enrolled in the study had been assigned to clinicians who left their agencies during the study. Of these families, $39 \%$ were identified as possibly experiencing service disruption, while $19 \%$ were identified as likely experiencing service disruption. Furthermore, most of the families $(n=3,60 \%)$ within this likely service disruption group reported an increase in child behavior problems over time, indicating poor therapeutic outcomes.

Although this descriptive analysis is by no means definitive, it is consistent with a prior study that reported very little effect of clinician turnover on outcomes for youth receiving substance use treatment (Garner et al., 2013). Although poor therapeutic outcomes were noted for a subset of families, this group was a very small subset of the larger sample. However, other studies have shown a detrimental effect of clinician turnover on client outcomes for youth in foster care (Strolin-Goltzman et al., 2010) and for adults with chronic pain (Williams \& Potts, 2010). Given the dearth of studies directly examining this hypothesis and the inconsistent findings among the few existing studies, additional research is needed to better understand the extent to which clinician turnover impacts client outcomes.

One important factor to consider when examining the relation between clinician turnover and therapeutic outcomes within this sample is the highly manualized nature of PCIT. It is much easier for a new clinician to take over a case with a manualized intervention than it is for other non-manualized interventions. This is because the original clinician would have left an indication of which session had been completed last, and the new clinician could pick up exactly where the family had left of. Thus is it possible that therapeutic outcomes are less likely to be impacted by clinician turnover in a manualized intervention. This could help explain some of the 
discrepancies within the literature, as perhaps the two studies that noted detrimental effects of clinician turnover were not using manualized interventions (Strolin-Goltzman et al., 2010; Williams \& Potts, 2010).

\section{Strengths}

Several strengths of the current study warrant mentioning. First, as previously mentioned, this was one of the first studies to examine differences in the rates and predictors of turnover for different types of behavioral health staff. Previous studies have included higher-level staff such as supervisors in analyses with clinicians (Beidas et al., 2015) or have asked for administrator perspectives on barriers to training and EBT implementation (Herschell et al., 2009). However, this is the first study to explore predictors of turnover for staff members at these different levels separately. In a similar vein, this is one of the first studies to examine how different training methods might impact behavioral health staff turnover. Numerous studies have focused on training outcomes in implementing EBTs (see Herschell, Kolko, Baumann, \& Davis, 2010) and have mentioned clinician dropout or turnover as hindering implementation efforts (Herschell et al., 2009; David J. Kolko et al., 2012). However, no studies to date have explored the possibility that turnover rates might vary across different EBT training conditions.

Additionally, the inclusion of both job role and training condition within the current study resulted in unique and interesting findings regarding the influence of training methods on staff members at higher levels of the organizational hierarchy. In most studies of both behavioral health turnover (e.g., Aarons et al., 2009; Bukach et al., 2015; Sheidow et al., 2007) and training outcomes (Herschell et al., 2010), focus has been on direct service providers. This focus has occurred for a number of important reasons. First, direct service providers are generally the only staff members involved EBT training and implementation, with the noted exception of learning 
collaborative training models. Second, their involvement in training means that they are the ones for whom significant costs associated with training are incurred (Cook et al., 2009). Third, they are largely responsible for client outcomes, as they are directly implementing the intervention with the clients. Relatedly, they are at a higher risk for burnout as a result of regular interaction with possibly challenging cases (Maslach, Schaufeli, \& Leiter, 2001). However, this focus on clinicians is a limitation within both the turnover literature and the training literature, given that poor organizational and administrative support is often implicated as both a predictor of greater clinician turnover (Allen et al., 2003) and as a barrier to EBT implementation (Aarons, Fettes, et al., 2009; Beidas et al., 2015). As one of the first studies to examine both different job roles and different training conditions in the context of behavioral health turnover, the findings from the current study make an important contribution to understanding factors that can impact EBT implementation efforts.

An additional strength of the current study was the examination of client outcomes relative to clinician turnover. One of the primary reasons that researchers have focused on clinician turnover is that is has long been hypothesized to negatively impact client outcomes; however, very few studies have directly examined this hypothesis (Garner et al., 2013; StrolinGoltzman et al., 2010; Williams \& Potts, 2010). Although the analysis presented in the current study was more descriptive in nature, it did indicate that some clients, albeit a small proportion, are likely to experience service disruption and negative outcomes as a result of clinician turnover.

\section{Limitations}

Despite the strengths of the current study, some limitations are worth noting. First, turnover is a complex phenomenon that is influenced by numerous variables which interact in 
various ways. As such, complex analyses requiring substantial statistical power are generally required to obtain a nuanced understanding of factors that predict turnover. Although efforts were made within the current study to carefully select variables of interest in order to maximize power, it is possible that excluded variables may have contributed more to understanding turnover within the current sample. This may be particularly true for clinicians, the group for whom no significant predictors of turnover were identified. Many turnover researchers have moved toward structural equation modeling (SEM) as the analysis of choice for understanding clinician turnover (Aarons \& Sawitzky, 2006; Aarons, Sommerfield, \& Wilging, Cathleen, 2011; Kim \& Lee, 2009; McGowan et al., 2009). SEM enables researchers to more precisely model both direct and indirect relations between unidimensional and multidimensional predictors of turnover. Although this type of modeling is ideal for complex processes like turnover, it requires a large sample size that was not available in the current study.

The small sample size within the current study was further limited by the nested structure of the data. Although HLM is designed to account for this nested structure, statistical power within HLM is determined by the number of groups at the highest level - in this case, the 50 agencies at level two. It is possible that, despite best efforts to maximize power within the current study, the sample size was too small to identify significant predictors, especially for clinicians.

Sample size and nesting were also limitations when assessing the relation between clinician turnover and client outcomes. As clients within the current study were nested within clinicians, HLM would have been the analysis of choice if inferential analyses had been possible. However, only 26 of the 110 enrolled families had been assigned to clinicians who left their agencies, which would not have provided sufficient power to run HLM. 
Another limitation of the current study was that it used data collected from a larger parent study. Although the parent study was not designed specifically to assess turnover, special considerations were taken to assess variables associated with turnover. Unfortunately, some key variables that have been identified in previous studies, such as salary (Beidas et al., 2015; BenDror, 1994a; Bukach et al., 2015), promotional chances (Griffeth et al., 2000), and number of children (Griffeth et al., 2000) were not collected in the parent study and thus could not be considered for inclusion in the current analyses. Additionally, education had to be excluded from analyses due to a lack of variability.

\section{Future Research}

The unique emphasis within the current study on: (a) turnover at various levels of the organizational hierarchy and (b) the influence of training methods on turnover has provided a number of important directions for future research. First, the current study presented evidence that the multidimensional construct of organizational climate predicts turnover for supervisors and administrators. Given that this is one of the first studies to identify a predictor of turnover for staff members in higher positions within a behavioral health agency, future research may consider breaking down the construct of organizational climate to determine if there are any particular indicator variables that drive this relation (e.g., job stress or burnout).

In addition, as organizational and administrative support has been identified as important to clinician turnover (Aarons et al., 2011; Allen et al., 2003), more researchers should consider including staff members at higher levels of the organizational hierarchy in studies on turnover. Although some research has examined the influence of clinician-reported organizational leadership (Aarons et al., 2011) and supervisory practices (Kim \& Lee, 2009) on clinician turnover, no studies to date have assessed whether administrator or supervisor reports of their 
own leadership practices, job stress, or other variables might trickle down and impact clinicians. This may be an important angle to consider in light of the current findings in which organizational climate influenced supervisor and administrator turnover and differences across trainings conditions approached significance, but no predictors of clinician turnover were identified.

One somewhat surprising finding within the current study was the low rate of annual turnover ( $8 \%$ for the full sample) compared to rates that are typically reported in behavioral health settings (30-50\%; Bjorklund et al., 2009; Bukach et al., 2015; Garner et al., 2012; Selden, 2010). Other researchers have also noted lower rates of turnover for clinicians using EBTs compared with those using TAU (Aarons, Fettes, et al., 2009; Beidas et al., 2015; Kolko et al., 2012). The hypothesis that EBT use reduces clinician turnover should be directly examined in future research. If this hypothesis is supported, researchers should seek to understand what it is about EBTs that reduce turnover. One hypothesis is that EBTs provide clinicians with more effective methods to treat their clients, resulting in quicker positive outcomes, fewer adverse events, and reduced clinician burnout (Aarons, Sommerfeld, et al., 2009). An alternative hypothesis is that most EBTs require some ongoing support or fidelity monitoring, which may be perceived by clinicians as extra organizational support (Aarons, Sommerfeld, et al., 2009). Given the mounting evidence that clinicians implementing EBTs have lower rates of turnover, future research should consider investigating the mechanism by which this relation occurs.

Although the 12-month rate of turnover within the current study was comparatively low, the 24-month rate of turnover (30\%) was more consistent with previous research (Aarons et al., 2011; Glisson, Schoenwald, et al., 2008; Sheidow et al., 2007). As previously mentioned, it is possible that clinicians in the current study remained at their agencies just long enough to 
become certified in PCIT and left just after completing training. Future research should consider addressing this hypothesis, perhaps through survival analyses to determine if there are different predictors of turnover depending on when clinicians change agencies or jobs.

Another unique contribution of the current study was the examination of clinician turnover related to client outcomes. Although the analysis presented within the current study had many limitations, it clearly supported the need for future research on this topic. Questions remain regarding the influence of clinician turnover on client outcomes, despite the prevalence of this assumption (e.g., Albizu-García, Ríos, Juarbe, \& Alegría, 2004; Kim \& Stoner, 2008; Mor Barak et al., 2001; Sheidow et al., 2007; Woltmann et al., 2008). The few studies that have directly examined this hypothesis have reported inconsistent results (Garner et al., 2013; StrolinGoltzman et al., 2010; Williams \& Potts, 2010). One possible explanation for the lack of research in this area is the difficulty in assessing it. Behavioral health clients and clinicians are two groups with traditionally high rates of dropout (de Haan, Boon, de Jong, Hoeve, \& Vermeiren, 2013) and turnover (Ben-Dror, 1994), which makes it difficult to obtain large enough sample sizes for the requisite analyses.

An additional factor for future researchers on this topic to consider is the possibility that behavioral health agencies have contingency plans for how to handle clients when a clinician leaves. It would be important for future researchers investigating the association between clinician turnover and client outcomes to know the extent to which different agencies have such contingency plans, and what such plans might look like. If contingency plans are standard practice in most agencies, it is possible that therapeutic outcomes may not actually be drastically impacted by clinician turnover, and researchers should focus on the other issues related to turnover (e.g., higher costs, increased stress for other staff members, etc.). 


\section{Implications}

Results of the current study supported the possibility of a protective effect of EBT use on clinician turnover. As such, one possible strategy for reducing rates of clinician turnover is to increase the availability of and organizational support for EBT trainings. More widespread EBT training could reduce clinician turnover in two possible ways. First, the EBT training may have a direct reduction on turnover as a result of mechanism underlying the protective effect (e.g., better client outcomes or increased support through fidelity monitoring - additional research is needed to more fully understand the cause). Second, more clinicians trained in EBTs across the entire behavioral health workforce would decrease demand, thus decreasing clinician motivation to leave an agency for a new opportunity after receiving EBT training and indirectly reducing turnover.

However, training methods should be selected carefully, as results of the current study indicate that they may have an impact on more than just clinician knowledge and skill. Learning collaborative training models, which were designed to increase organizational support and readiness for implementing an EBT (National Research Council and Institute of Medicine, 2009), may actually backfire if supervisors and administrators are not on board with the additional responsibilities required of them. Results of the larger parent study will provide insight into the cost effectiveness and implementation outcomes for each training method. These results must be considered in conjunction with results of the current study when determining which training method produced the most favorable outcomes. Individuals interested in the training of behavioral health providers must be aware of such evidence and carefully craft training methods that will provide optimal outcomes across numerous critical domains. 
Additionally, organizational climate should be carefully assessed prior to introducing EBT training. As negative climates can result in increased turnover, both for clinicians (Aarons \& Sawitzky, 2006; Beidas et al., 2015) and for supervisors and administrators (results of the current study), it is possible that steps should be taken to remediate these issues before clinicians receive training in order to reduce the likelihood that they will later leave the agency. In fact, recent research has focused on organizational interventions to improve the likelihood that clinicians will attend training workshops (Glisson et al., 2016). Although this study did not directly examine the effect of the organizational intervention on clinician turnover, it is an important first step in understanding ways to improve organizations and promote employee retention.

\section{Conclusion}

Staff turnover is an important problem within the behavioral health field, especially given the recent focus on increasing the number of clinicians trained in EBTs. Results of the current study are consistent with other findings that note a possible protective effect of EBTs on clinician turnover. However, results also indicate that both training method and organization climate influence turnover rates for higher-ranking staff members. Although turnover is already a complex process, future research should focus on the potential trickle-down effect of job stress from administrators and supervisors to clinicians. Improved understanding of the interrelations between clinician, supervisor, and administrator factors may help to create training methods that promote improved intervention implementation and sustainability, as well as organizational interventions to increase an agency's readiness to implement a new intervention. 


\section{References}

Aarons, G. A., Fettes, D. L., Flores, L. E., \& Sommerfeld, D. H. (2009). Evidence-based practice implementation and staff emotional exhaustion in children's services. Behaviour Research and Therapy, 47(11), 954-60. http://doi.org/10.1016/j.brat.2009.07.006

Aarons, G. A., \& Palinkas, L. A. (2007). Implementation of evidence-based practice in child welfare: Service provider perspectives. Administration and Policy in Mental Health and Mental Health Services Research, 34(4), 411-419. http://doi.org/10.1007/s10488-007-01213

Aarons, G. A., \& Sawitzky, A. C. (2006). Organizational climate partially mediates the effect of culture on work attitudes and staff turnover in mental health services. Administration and Policy in Mental Health and Mental Health Services Research, 33(3), 289-301. http://doi.org/10.1007/s10488-006-0039-1

Aarons, G. A., Sommerfeld, D. H., Hecht, D. B., Silovsky, J. F., \& Chaffin, M. J. (2009). The impact of evidence-based practice implementation and fidelity monitoring on staff turnover: Evidence for a protective effect. Journal of Consulting and Clinical Psychology, 77(2), 270-80. http://doi.org/10.1037/a0013223

Aarons, G. A., Sommerfield, D. H., \& Wilging, Cathleen, E. (2011). The soft underbelly of system change: The role of leadership in organization climate in turnover during statewide behavioral health reform. Psychological Services, 8(4), 269-281. http://doi.org/10.1037/a002619. 
Achenbach, T. M., Dumenci, L., \& Rescorla, L. A. (2002). Ten-year comparisons of problems and competencies for national samples of youth: Self, parent, and teacher reports. Journal of Emotional and Behavioral Disorders, 10(4), 194-203.

http://doi.org/10.1177/10634266020100040101

Adair, C. E., McDougall, G. M., Mitton, C. R., Joyce, A. S., Wild, T. C., Gordon, A., ... Beckie, A. (2005). Continuity of care and health care costs among persons with severe mental illness. Psychiatric Services, 56(9), 1070-1076. http://doi.org/10.1176/appi.ps.56.9.1070

Albizu-García, C. E., Ríos, R., Juarbe, D., \& Alegría, M. (2004). Provider turnover in public sector managed mental health care. The Journal of Behavioral Health Services \& Research, 31(3), 255-65. http://doi.org/00075484-200407000-00003 [pii]

Allen, D. G., Shore, L. M., \& Griffeth, R. W. (2003). The role of perceived organizational support and supportive human resource practices in the turnover process. Journal of Management, 29(1), 99-118. http://doi.org/10.1177/014920630302900107

APA. (2005). Report of the 2005 presidential task force on evidence-based practice.

Beidas, R. S., Marcus, S., Wolk, C. B., Powell, B., Aarons, G. A., Evans, A. C., ... Mandell, D. S. (2015). A prospective examination of clinician and supervisor turnover within the context of implementation of evidence-based practices in a publicly-funded mental health system. Administration and Policy in Mental Health and Mental Health Services Research. http://doi.org/10.1007/s10488-015-0673-6

Ben-Dror, R. (1994). Employee turnover in community mental health organization: A developmental stages study. Community Mental Health Journal, 30(3), 243-257. 
Bjorklund, R. W., Monroe-DeVita, M., Reed, D., Toulon, A., \& Morse, G. (2009). Washington State's initiative to disseminate and implement high-fidelity ACT teams. Psychiatric Services, 60(1), 24-27. http://doi.org/10.1176/appi.ps.60.1.24

Blankertz, L. E., \& Robinson, S. E. (1997). Turnover intentions of community mental health workers in psychosocial rehabilitation services. Community Mental Health Journal, 33(6), 517-29. http://doi.org/10.1023/A:1025000703487

Bukach, A. M., Ejaz, F. K., Dawson, N., \& Gitter, R. J. (2015). Turnover among community mental health workers in Ohio. Administration and Policy in Mental Health and Mental Health Services Research. http://doi.org/10.1007/s10488-015-0706-1

Catania, L. S., Hetrick, S. E., Newman, L. K., \& Purcell, R. (2011). Prevention and early intervention for mental health problems in 0-25 year olds: Are there evidence-based models of care? Advances in Mental Health, 10(1), 6-19. http://doi.org/10.5172/jamh.2011.10.1.6

Cavaleri, M. A., Gopalan, G., Mckay, M. M., Appel, A., Jr, W. M. B., Bigley, M. F., ... Sher, T. (2006). Impact of a learning collaborative to improve child mental health service use among low-income urban youth and families. Children, 2(2), 67-79.

Christian, A. S., Niec, L. N., Acevedo-Polakovich, I. D., \& Kassab, V. A. (2014). Dissemination of an evidence-based parenting program: Clinician perspectives on training and implementation. Children and Youth Services Review, 43, 8-17. http://doi.org/10.1016/j.childyouth.2014.04.005

Cook, J. M., Biyanova, T., \& Coyne, J. C. (2009). Barriers to adopotion of new treatments: An internet study of practicing community psychotherapists. Administration and Policy in Mental Health and Mental Health Services Research, 36(2), 83-90. http://doi.org/10.1007/s10488-008-0198-3 
Cropanzano, R., Rupp, D. E., \& Byrne, Z. S. (2003). The relationship of emotional exhaustion to work attitudes, job performance, and organizational citizenship behaviors. Journal of Applied Psychology, 88(1), 160-169. http://doi.org/10.1037/0021-9010.88.1.160

de Haan, A. M., Boon, A. E., de Jong, J. T. V. M., Hoeve, M., \& Vermeiren, R. R. J. M. (2013). A meta-analytic review on treatment dropout in child and adolescent outpatient mental health care. Clinical Psychology Review, 33(5), 698-711. http://doi.org/10.1016/j.cpr.2013.04.005

Dopp, A. R., Hanson, R. F., Saunders, B. E., Dismuke, C. E., \& Moreland, A. D. (2017). Community-based implementation of trauma-focused interventions for youth: Economic impact of the learning collaborative model. Psychological Services, 14(1), 57-65. http://doi.org/10.1037/ser0000131

Dougherty, D., Schiff, J., \& Mangione-Smith, R. (2011). The children's health insurance program reauthorization act quality measures initiatives: Moving forward to improve measurement, care, and child and adolescent outcomes. Academic Pediatrics, 11(3 SUPPL.), S1-S10. http://doi.org/10.1016/j.acap.2011.02.009

Ellett, A. J., Ellis, J. I., Westbrook, T. M., \& Dews, D. (2007). A qualitative study of 369 child welfare professionals' perspectives about factors contributing to employee retention and turnover. Children and Youth Services Review, 29(2), 264-281. http://doi.org/10.1016/j.childyouth.2006.07.005

Garner, B. R., Funk, R. R., \& Hunter, B. D. (2013). The relationship between clinician turnover and adolescent treatment outcomes: An examination from the client perspective. Journal of Substance Abuse Treatment, 44(4), 444-448. http://doi.org/http://dx.doi.org/10.1016/j.jsat.2012.09.004 
Garner, B. R., Hunter, B. D., Modisette, K. C., Ihnes, P. C., \& Godley, S. H. (2012). Treatment staff turnover in organizations implementing evidence-based practices: Turnover rates and their association with client outcomes. Journal of Substance Abuse Treatment, 42(2), 134142. http://doi.org/10.1016/j.jsat.2011.10.015.

Glisson, C., \& James, L. R. (2002). The cross-level effects of culture and climate in human service teams. Journal of Organizational Behavior, 23(6), 767-794. http://doi.org/10.1002/job.162

Glisson, C., Landsverk, J., Schoenwald, S., Kelleher, K., Hoagwood, K. E., Mayberg, S., ... Palinkas, L. (2008). Assessing the Organizational Social Context (OSC) of mental health services: Implications for research and practice. Administration and Policy in Mental Health and Mental Health Services Research, 35(1-2), 98-113. http://doi.org/10.1007/s10488-007$0148-5$

Glisson, C., Schoenwald, S. K., Kelleher, K., Landsverk, J., Hoagwood, K. E., Mayberg, S., \& Green, P. (2008). Therapist turnover and new program sustainability in mental health clinics as a function of organizational culture, climate, and service structure. Administration and Policy in Mental Health and Mental Health Services Research, 35(1-2), 124-133. http://doi.org/10.1007/s10488-007-0152-9

Glisson, C., Williams, N. J., Hemmelgarn, A., Proctor, E., \& Green, P. (2016). Increasing clinicians' EBT exploration and preparation behavior in youth mental health services by changing organizational culture with ARC. Behaviour Research and Therapy, 76, 40-46. http://doi.org/10.1016/j.brat.2015.11.008 
Gray, A. M., \& Phillips, V. L. (1994). Turnover, age and length of service: a comparison of nurses and other staff in the National Health Service. Journal of Advanced Nursing, 19(4), 819-827. http://doi.org/10.1111/j.1365-2648.1994.tb01155.x

Griffeth, R. W. ., Hom, P. W., \& Gaertner, S. (2000). A meta-analysis of antecedents and correlates of employee turnover: update, moderator tests, and research implications for the next millennium. Journal of Management, 26(3), 463-488. http://doi.org/10.1177/014920630002600305

Herschell, A. D., Kogan, J. N., Celedonia, K. L., Gavin, J. G., \& Stein, B. D. (2009). Understanding community mental health administrators' perspectives on Dialectical Behavior Therapy implementation. Psychiatric Services, 60(7), 989-92. http://doi.org/10.1176/appi.ps.60.7.989

Herschell, A. D., Kolko, D. J., Baumann, B. L., \& Davis, A. C. (2010). The role of therapist training in the implementation of psychosocial treatments: A review and critique with recommendations. Clinical Psychology Review, 30(4), 448-466. http://doi.org/10.1016/j.cpr.2010.02.005

Herschell, A. D., Kolko, D. J., Scudder, A. T., Taber-Thomas, S., Schaffner, K. F., Hiegel, S. A., ... Mrozowski, S. (2015). Protocol for a statewide randomized controlled trial to compare three training models for implementing an evidence-based treatment. Implementation Science, 10(1), 133. http://doi.org/10.1186/s13012-015-0324-z

IBM Corp. (2016). IBP SPSS Statistics for Windows, Version 24.0. Armonk, NY: IBM Corp. Iglehart, A. P. (1990). Turnover in the social services: turning over to the benefits. Social Service Review, 649-657. 
Ingersoll, R. M. (2001). Teacher turnover and teacher shortages : An organizational analysis. American Educational Research Journal, 38(3), 499-534. http://doi.org/10.3102/00028312038003499

Jackson, C. B., Brabson, L. A., Quetsch, L. B., \& Herschell, A. D. (n.d.). Web-based training methods for behavioral health providers: A systematic review. Clinical Psychology Review.

Kessler, R. C., Berglund, P., Demler, O., Jin, R., Merikangas, K. R., \& Walters, E. E. (2005). Lifetime prevalence and age-of-onset distributions of DSM-IV disorders in the National Comorbidity Survey replication. Archive of General Psychiatry, 62, 593-602. http://doi.org/10.1001/archpsyc.62.6.593

Kessler, R. C., \& Wang, P. S. (2008). The descriptive epidemiology of commonly occurring mental disorders in the United States. Annual Review of Public Health, 29, 115-129. http://doi.org/10.1146/annurev.publhealth.29.020907.090847

Kim, H., \& Lee, S. Y. (2009). Supervisory communication, burnout, and turnover intention among social workers in health care settings. Social Work in Health Care, 48(4), 364-385. http://doi.org/10.1080/00981380802598499

Kim, H., \& Stoner, M. (2008). Burnout and turnover intention among social workers: effects of role stress, job autonomy and social support. Administration in Social Work, 32(3), 5-25. http://doi.org/10.1080/03643100801922357

Knudsen, H. K., Ducharme, L. J., \& Roman, P. M. (2006). Counselor emotional exhaustion and turnover intention in therapeutic communities. Journal of Substance Abuse Treatment, 31(2), 173-180. http://doi.org/10.1016/j.jsat.2006.04.003 
Knudsen, H. K., Ducharme, L. J., \& Roman, P. M. (2007). Research participation and turnover intention: An exploratory analysis of substance abuse counselors. Journal of Substance Abuse Treatment, 33(2), 211-7. http://doi.org/10.1016/j.jsat.2006.12.013

Knudsen, H. K., Ducharme, L. J., \& Roman, P. M. (2008). Clinical supervision, emotional exhaustion, and turnover intention: A study of substance abuse treatment counselors in the Clinical Trials Network of the National Institute on Drug Abuse. Journal of Substance Abuse Treatment, 35(4), 387-395. http://doi.org/10.1016/j.jsat.2008.02.003

Kolko, D. J., Baumann, B. L., Herschell, A. D., Hart, J. A., Holden, E. A., \& Wisniewski, S. R. (2012). Implementation of AF-CBT by community practitioners serving child welfare and mental health: A randomized trial. Child Maltreatment, 17(1), 32-46. http://doi.org/10.1177/1077559511427346

Lambert, E. G., Hogan, N., \& Barton, S. M. (2001). The impact of job satisfaction on turnover intent: A test of a structural measurement model using a national sample of workers. Social Science Journal, 38(2), 233-250. http://doi.org/10.1016/S0362-3319(01)00110-0

Lanier, P., Kohl, P. L., Benz, J., Swinger, D., Moussette, P., \& Drake, B. (2011). Parent-Child Interaction Therapy in a community setting: Examining outcomes, attrition, and treatment Setting. Research on Social Work Practice, 21(6), 689-698. http://doi.org/10.1177/1049731511406551

Lavigne, J. V, Binns, H. J., Arend, R., Rosenbaum, D., Christoffel, K. K., Hayford, J. R., \& Gibbons, R. D. (1998). Psychopathology and health care use among preschool children: A retrospective analysis. Journal of the American Academy of Child \& Adolescent Psychiatry, 37(3), 262-270. http://doi.org/10.1097/00004583-199803000-00010 
Lehman, W. E. K., Greener, J. M., \& Simpson, D. D. (2002). Assessing organizational readiness for change. Journal of Substance Abuse Treatment, 22(4), 197-209. http://doi.org/10.1016/S0740-5472(02)00233-7

Lyon, A., \& Budd, K. (2010). A community mental health implementation of Parent-Child Interaction Therapy. Journal of Child and Family Studies, 19(5), 654-668. http://doi.org/10.1007/s10826-010-9353-z.A

Maslach, C., \& Jackson, S. (1981). The measurement of experienced burnout. Journal of Occupational Behavior, 2, 99-113. http://doi.org/10.1002/job.4030020205

Maslach, C., Schaufeli, W. B., \& Leiter, M. P. (2001). Job burnout. Annual Review of Psychology, 52, 397-422. http://doi.org/10.1146/annurev.psych.52.1.397

McGowan, B., Auerbach, C., \& Strolin-Goltzman, J. (2009). Turnover in the child welfare workforce: A different perspective. Journal of Social Service Research, 35(3), 228-235. http://doi.org/10.1080/01488370902900782

McNeil, C. B., \& Hembree-Kigin, T. L. (2010). Parent-Child Interaction Therapy (2nd ed.). New York, NY: Springer Science \& Business Media.

McNulty, T. L., Oser, C. B., \& Johnson, J. A. (2007). Counselor turnover in substance abuse treatment centers: An organizational-level analysis. Sociological Inquiry, 77(2), 166-193. http://doi.org/10.1111/j.1475-682X.2007.00186.x

Mor Barak, M. E., Nissly, J. A., \& Levin, A. (2001). Antecedents to retention and turnover among child welfare, social work, and other human service employees: What can we learn from past research? A review and metanalysis. Social Service Review, 75(4), 625-661. http://doi.org/10.1086/323166 
National Research Council and Institute of Medicine. (2009). Preventing Mental, Emotional, and Behavioral Disorders Among Young People: Progess and Possibilities. (M. E. O’Connell, T. Boat, \& K. E. Warner, Eds.). Washington, DC: National Academies Press.

Novins, D. K., Green, A. E., Legha, R. K., \& Aarons, G. A. (2013). Dissemination and implementation of evidence-based practices for child and adolescent mental health: A systematic review. Journal of the American Academy for Child Adolescent Psychiatry, 52(10), 1009-1025. http://doi.org/10.1016/j.biotechadv.2011.08.021.Secreted

Oppenheim, J., Stewart, W., Zoubak, E., Donato, I., Huang, L., \& Hudock, W. (2016).

Launching forward: the integration of behavioral health in primary care as a key strategy for promoting young child wellness. American Journal of Orthopsychiatry, 86(2), 124-131. http://doi.org/10.1037/ort0000149

PCIT International Inc. (n.d.). Get certified by PCIT International. Retrieved December 5, 2016, from http://www.pcit.org/pcit-certification.html

Pearl, E., Thieken, L., Olafson, E., Boat, B., Connelly, L., Barnes, J., \& Putnam, F. (2012). Effectiveness of community dissemination of parent-child interaction therapy. Psychological Trauma: Theory, Research, Practice, and Policy, 4(2), 204-213. http://doi.org/10.1037/a0022948

Pritchard, R. D., \& Karasick, B. W. (1973). The effects of organizational climate on managerial job performace and job satisfaction. Organizational Behavior and Human Performance, 9, 126-146. http://doi.org/10.1016/0030-5073(73)90042-1

Raudenbush, S. W., Bryk, A. S., \& Congdon, R. T. (2011). HLM 7.00 for Windows. Lincolnwood, IL: Scientific Software International, Inc. 
Rich, B. A., \& Eyberg, S. M. (2001). Accuracy of assessment: The discriminative and predictive power of the Eyberg Child Behavior Inventory. Ambulatory Child Health, 7, 249-257. http://doi.org/10.1046/j.1467-0658.2001.00141.x.X

Rollins, A. L., Salyers, M. P., Tsai, J., \& Lydick, J. M. (2010). Staff turnover in statewide implementation of ACT: relationship with ACT fidelity and other team characteristics. Administration and Policy in Mental Health and Mental Health Services Research, 37(5), 417-26. http://doi.org/10.1007/s10488-009-0257-4

Selden, D. R. (2010). The effects of staff turnover on psychiatric rehabilitation programs. Psychiatric Rehabilitation Journal, 34(1), 71-73. http://doi.org/10.2975/34.1.2010.71.73

Sheidow, A. J., Schoenwald, S. K., Wagner, H. R., Allred, C. a, \& Burns, B. J. (2007). Predictors of workforce turnover in a transported treatment program. Administration and Policy in Mental Health and Mental Health Services Research, 34(1), 45-56. http://doi.org/10.1007/s10488-006-0061-3

Shore, B. A., Iwata, B. A., Vollmer, T. R., Lerman, D. C., \& Zarcone, J. R. (1995). Pyramidal staff training in the extension of treatment for severe behavior disorders. Journal of Applied Behavior Analysis, 28(3), 323-332.

Steel, R. P., \& Ovalle, N. K. (1984). A review and meta-analysis of research on the relationship between behavioral intentions and employee turnover. Journal of Applied Psychology, 69(4), 673-686. http://doi.org/10.1037/0021-9010.69.4.673

Strolin-Goltzman, J., Kollar, S., \& Trinkle, J. (2010). Listening to the voices of children in foster care: youths speak out about child welfare workforce turnover and selection. Social Work, 55(1), 47-53. http://doi.org/10.1093/sw/55.1.47

Tabachnick, B. G., \& Fidell, L. S. (2014). Using Multivariate Statistics (Sixth). London, UK. 
Tett, R. P., \& Meyer, J. P. (1993). Job satisfaction, organizational commitment, turnover intention, and turnover: path analyses based on meta-analytic findings. Personnel Psychology, 46(2), 259-293. http://doi.org/10.1111/j.1744-6570.1993.tb00874.x

Thomas, R., \& Zimmer-Gembeck, M. J. (2007). Behavioral outcomes of Parent-Child Interaction Therapy and Triple P - Positive Parenting Program: A review and meta-analysis. Journal of Abnormal Child Psychology, 35(3), 475-495. http://doi.org/10.1007/s10802-007-9104-9

Williams, A. C. D. C., \& Potts, H. W. W. (2010). Group membership and staff turnover affect outcomes in group CBT for persistent pain. Pain, 148(3), 481-6. http://doi.org/10.1016/j.pain.2009.12.011

Woltmann, E. M., Whitley, R., McHugo, G. J., Brunette, M., Torrey, W. C., Coots, L., ... Drake, R. E. (2008). The role of staff turnover in the implementation of evidence-based practices in mental health care. Psychiatric Services (Washington, D.C.), 59(7), 732-7. http://doi.org/10.1176/appi.ps.59.7.732

Wright, T. A, \& Cropanzano, R. (1998). Emotional exhaustion as a predictor of job performance and voluntary turnover. The Journal of Applied Psychology, 83(3), 486-493. http://doi.org/10.1037/0021-9010.83.3.486 
Table 1

Demographics for Clinicians, Supervisors, and Administrators

\begin{tabular}{|c|c|c|c|}
\hline & $\begin{array}{c}\text { Clinicians }^{\mathrm{a}} \\
\mathrm{n}(\%)\end{array}$ & $\begin{array}{c}\text { Supervisors }^{\mathrm{b}} \\
\mathrm{n}(\%)\end{array}$ & $\begin{array}{c}\text { Administrators }^{\mathrm{c}} \\
\mathrm{n}(\%)\end{array}$ \\
\hline \multicolumn{4}{|l|}{ Gender } \\
\hline Male & $16(16.0 \%)$ & $10(20.0 \%)$ & $19(38.0 \%)^{* a, b}$ \\
\hline Female & $84(84.0 \%)^{* c}$ & $40(80.0 \%)^{* \mathrm{c}}$ & $31(62.0 \%)$ \\
\hline \multicolumn{4}{|l|}{ Race } \\
\hline African American & $5(5.0 \%)$ & $3(6.0 \%)$ & $1(2.0 \%)$ \\
\hline Asian & $1(1.0 \%)$ & $0(0.0 \%)$ & $1(2.0 \%)$ \\
\hline Caucasian & $91(91.0 \%)$ & $44(94.0 \%)$ & $45(90.0 \%)$ \\
\hline Native American/Alaska Native & $3(3.0 \%)$ & $0(0.0 \%)$ & $0(0.0 \%)$ \\
\hline Native Hawaiian/Pacific Islander & $3(3.0 \%)$ & $0(0.0 \%)$ & $0(0.0 \%)$ \\
\hline Not Reported & $3(3.0 \%)$ & $0(0.0 \%)$ & $3(6.0 \%)$ \\
\hline \multicolumn{4}{|l|}{ Ethnicity } \\
\hline Hispanic/Latino & $8(8.0 \%)$ & $5(10.0 \%)$ & $3(6.0 \%)$ \\
\hline Not Hispanic/Latino & $92(92.0 \%)$ & $45(90.0 \%)$ & $47(94.0 \%)$ \\
\hline \multicolumn{4}{|l|}{ Education Level } \\
\hline Some college & $0(0.0 \%)$ & $0(0.0 \%)$ & $2(4.0 \%)$ \\
\hline Bachelor's degree & $0(0.0 \%)$ & $0(0.0 \%)$ & $2(4.0 \%)$ \\
\hline Some graduate work & $0(0.0 \%)$ & $0(0.0 \%)$ & $1(2.0 \%)$ \\
\hline Master's degree & $92(92.0 \%)^{* \mathrm{~b}, \mathrm{c}}$ & $41(82.0 \%)$ & $34(68.0 \%)$ \\
\hline Doctoral degree & $8(8.0 \%)$ & $9(18.0 \%)$ & $10(20.0 \%)$ \\
\hline \multicolumn{4}{|l|}{ Degree Type } \\
\hline Education & $2(2.0 \%)$ & $4(8.0 \%)$ & $3(6.0 \%)$ \\
\hline Medicine & $4(4.0 \%)$ & $1(2.0 \%)$ & $3(6.0 \%)$ \\
\hline Psychology & $37(37.0 \%)$ & $16(32.0 \%)$ & $11(22.0 \%)$ \\
\hline Social Work & $31(31.0 \%)$ & $18(36.0 \%)$ & $16(32.0 \%)$ \\
\hline Other & $26(26.0 \%)$ & $11(22.0 \%)$ & $15(30.0 \%)$ \\
\hline \multicolumn{4}{|l|}{ Condition } \\
\hline Learning Collaborative & $34(34.0 \%)$ & $17(34.0 \%)$ & $17(34.0 \%)$ \\
\hline Cascading Model & $32(32.0 \%)$ & $16(32.0 \%)$ & $16(32.0 \%)$ \\
\hline Distance Education & $34(34.0 \%)$ & $17(34.0 \%)$ & $17(34.0 \%)$ \\
\hline \multicolumn{4}{|l|}{ Assessment Method } \\
\hline Online & $86(86.0 \%)$ & $73(86.0 \%)$ & $\mathrm{n} / \mathrm{a}$ \\
\hline \multirow[t]{2}{*}{ Paper } & $14(14.0 \%)$ & $7(14.0 \%)$ & $\mathrm{n} / \mathrm{a}$ \\
\hline & $M(S D)$ & $M(S D)$ & $M(S D)$ \\
\hline Age & $39.04(10.04)$ & $44.77(9.48)^{+\mathrm{a}}$ & $48.51(8.86)^{+a}$ \\
\hline Years in human services industry & $11.42(8.20)$ & $18.26(8.60)^{+a}$ & $22.18(8.53)^{+a}$ \\
\hline Years at agency & $4.82(5.61)$ & $7.20(5.45)^{+\mathrm{a}}$ & $11.55(8.40)^{+\mathrm{a}}$ \\
\hline
\end{tabular}

Note: $* p<0.05 ;+p<0.01$

Superscripts indicate the group with which significant differences were noted for pairwise comparisons. 
Table 2

Demographics for Families

\begin{tabular}{|c|c|c|}
\hline & $\begin{array}{c}\text { Children } \\
\mathrm{n}(\%)\end{array}$ & $\begin{array}{c}\text { Parents } \\
\mathrm{n}(\%)\end{array}$ \\
\hline \multicolumn{3}{|l|}{ Gender } \\
\hline Male & $67(61.0 \%)$ & $4(3.6 \%)$ \\
\hline Female & $43(39.0 \%)$ & $106(96.4 \%)$ \\
\hline \multicolumn{3}{|l|}{ Race } \\
\hline African American & $28(25.5 \%)$ & $28(25.5 \%)$ \\
\hline Asian & $1(0.9 \%)$ & $1(0.9 \%)$ \\
\hline Caucasian & $75(68.1 \%)$ & $75(68.1 \%)$ \\
\hline Native American/Alaska Native & $3(2.7 \%)$ & $3(2.7 \%)$ \\
\hline Native Hawaiian/Pacific Islander & $0(0.0 \%)$ & $0(0.0 \%)$ \\
\hline Not Reported & $3(2.7 \%)$ & $3(2.7 \%)$ \\
\hline \multicolumn{3}{|l|}{ Ethnicity } \\
\hline Hispanic/Latino & $15(13.6 \%)$ & $14(12.7 \%)$ \\
\hline Not Hispanic/Latino & $95(86.4 \%)$ & $96(87.3 \%)$ \\
\hline \multicolumn{3}{|l|}{ Relationship to Child } \\
\hline Biological Mother & -- & $99(90.0 \%)$ \\
\hline Biological Father & -- & $4(3.6 \%)$ \\
\hline Adoptive Mother & -- & $4(3.6 \%)$ \\
\hline Grandmother & -- & $3(2.7 \%)$ \\
\hline \multicolumn{3}{|l|}{ Assessment Method } \\
\hline Online & -- & $67(60.9 \%)$ \\
\hline Phone & -- & $13(11.8 \%)$ \\
\hline Paper & -- & $30(27.3 \%)$ \\
\hline \multicolumn{3}{|l|}{ Education } \\
\hline Less than high school & -- & $8(7.3 \%)$ \\
\hline Some high school & -- & $14(12.7 \%)$ \\
\hline High school graduate/GED & -- & $35(31.8 \%)$ \\
\hline Some college & -- & $18(16.4 \%)$ \\
\hline Associate degree & -- & $9(8.2 \%)$ \\
\hline Bachelor's degree & -- & $6(5.5 \%)$ \\
\hline Graduate/professional education & -- & $10(9.1 \%)$ \\
\hline Did not report & & $10(9.1 \%)$ \\
\hline \multicolumn{3}{|l|}{ Household Income } \\
\hline$\$ 14,999$ or less & -- & $42(38.1 \%)$ \\
\hline$\$ 15,000$ to $\$ 29,999$ & -- & $22(20.0 \%)$ \\
\hline$\$ 30,000$ to $\$ 49,999$ & -- & $8(7.3 \%)$ \\
\hline$\$ 50,000$ to $\$ 74,999$ & -- & $6(5.5 \%)$ \\
\hline$\$ 75,000$ or more & -- & $9(8.2 \%)$ \\
\hline Did not report & & $23(20.9 \%)$ \\
\hline \multirow[t]{2}{*}{ Age } & $M(S D)$ & $M(S D)$ \\
\hline & $5.26(1.63)$ & $32.29(8.18)$ \\
\hline
\end{tabular}


Table 3

Rates of Turnover

\begin{tabular}{lcc}
\hline & 12 Month & 24 Month \\
\hline & $N(\%)$ & $N(\%)$ \\
Full Sample & $16(8.0 \%)$ & $59(29.5 \%)$ \\
Clinicians & $11(11 \%)$ & $31(31.0 \%)$ \\
Supervisors & $3(6.0 \%)$ & $15(30.0 \%)$ \\
Administrators & $2(4.0 \%)$ & $13(26.0 \%)$ \\
\hline
\end{tabular}


Table 4

Rates of Turnover by Training Condition

\begin{tabular}{lccc|ccc}
\hline \multicolumn{3}{c|}{12 Month } & \multicolumn{3}{c}{ 24 Month } \\
\hline & LC & CM & DE & LC & CM & DE \\
Full Sample & $4(6.3 \%)$ & $3(4.7 \%)$ & $9(13.2 \%)$ & $25(41.7 \%)$ & $14(21.9 \%)$ & $20(29.9 \%)$ \\
Clinicians & $2(6.7 \%)$ & $3(9.4 \%)$ & $6(17.6 \%)$ & $10(33.3 \%)$ & $32(28.1 \%)$ & $33(36.4 \%)$ \\
Supervisors & $1(5.9 \%)$ & $0(0.0 \%)$ & $2(11.8 \%)$ & $8(53.3 \%)$ & $3(18.8 \%)$ & $4(23.5 \%)$ \\
Administrators & $1(5.9 \%)$ & $0(0.0 \%)$ & $1(5.9 \%)$ & $7(46.7 \%)$ & $2(12.5 \%)$ & $4(23.5 \%)$ \\
\hline
\end{tabular}

LC - Learning Collaborative; CM - Cascading Model; DE - Distance Education 
Table 5

Bivariate Correlations between Predictor Variables

\begin{tabular}{|c|c|c|c|c|c|c|}
\hline \multirow[b]{2}{*}{ Variables } & \multicolumn{6}{|c|}{ Clinicians $(n=100)$} \\
\hline & $M(S D)$ & 1 & 2 & 3 & 4 & 5 \\
\hline 1. Age & $39.04(10.04)$ & -- & & & & \\
\hline 2. Salary & $45,039(11,574)$ & $0.41 * *$ & -- & & & \\
\hline 3. Tenure w/agency & $4.82(5.61)$ & $0.32 * *$ & 0.20 & -- & & \\
\hline 4. Org. Climate & $34.53(4.90)$ & 0.15 & -0.13 & $0.33 * *$ & -- & \\
\hline \multirow[t]{3}{*}{ 5. Org. Culture } & $34.17(5.52)$ & 0.13 & -0.15 & $0.20 *$ & $0.73 * *$ & -- \\
\hline & \multicolumn{6}{|c|}{ Supervisors and Administrators $(n=100)$} \\
\hline & $M(S D)$ & 1 & 2 & 3 & 4 & \\
\hline 1. Age & $46.62(9.37)$ & -- & & & & \\
\hline 2. Tenure w/agency & $9.35(7.36)$ & $0.37 * *$ & -- & & & \\
\hline 3. Org. Climate & $37.02(3.68)$ & 0.07 & 0.05 & -- & & \\
\hline 4. Org. Culture & $37.00(4.15)$ & 0.09 & 0.08 & $0.56 * *$ & -- & \\
\hline
\end{tabular}


Table 6

Predictors of Turnover

\begin{tabular}{llll}
\hline & & \multicolumn{3}{c}{ Clinician - Demographic Predictors } \\
\hline Variable & $B$ & $S E(B)$ & $\operatorname{Exp} B$ \\
Age & -0.01 & 0.03 & 0.99 \\
Salary & 0.00 & 0.00 & 1.00 \\
Tenure & -0.60 & 0.66 & 0.56 \\
\hline
\end{tabular}

Clinician - Organizational Predictors

Training Condition not Included

\begin{tabular}{lllllllll}
\hline Variable & Coefficient & $S E$ & $t$ & $d f$ & Coefficient & $S E$ & $t$ & $d f$ \\
Org. Culture & -0.05 & 0.08 & -0.17 & 47 & -0.05 & 0.08 & -0.63 & 47 \\
Org. Climate & -0.02 & 0.07 & -0.35 & 47 & -0.03 & 0.07 & -0.37 & 47 \\
\hline
\end{tabular}

Supervisor and Administrator - Demographic Predictors

\begin{tabular}{llll}
\hline Variable & $B$ & $S E(B)$ & $\operatorname{Exp} B$ \\
Age & 0.03 & 0.03 & 1.03 \\
Tenure & -1.03 & 0.59 & 0.36 \\
\hline
\end{tabular}

Supervisor and Administrator - Organizational Predictors Training Condition not Included Training Condition Included

\begin{tabular}{lllllllll}
\hline Variable & Coefficient & $S E$ & $t$ & $d f$ & Coefficient & $S E$ & $t$ & $d f$ \\
Org. Culture & -0.02 & 0.07 & -0.35 & 47 & 0.03 & 0.08 & 0.36 & 47 \\
Org. Climate & $-0.14^{*}$ & 0.07 & -2.09 & 47 & $-0.16^{*}$ & 0.07 & -2.20 & 43 \\
\hline
\end{tabular}

$* p<0.05$ 


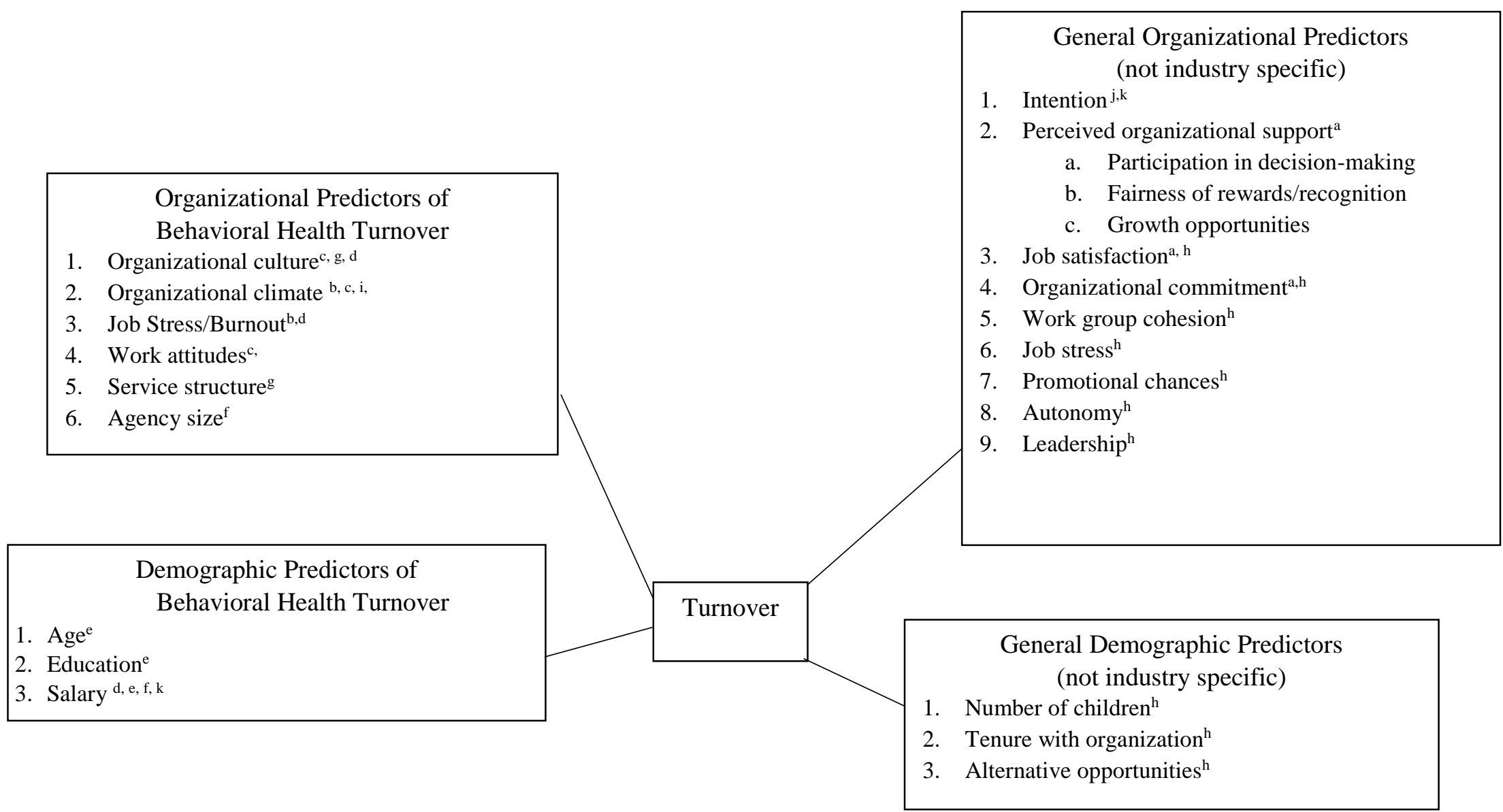

Figure 1. Chart showing significant direct predictors of employee turnover.

a) Allen, Lynn, \& Griffeth, 2003

b) Aarons et al., 2011

c) Aarons \& Sawitzky, 2006

d) Beidas et al., 2015

e) Ben-Dror, 1994

f) Bukach et al., 2015

g) Glisson et al., 2008

h) Griffeth, Hom, \& Gaertner, 2000

i) Sheidow et al., 2006

j) Steele \& Ovalle, 1984

k) Tett \& Meyer, 1993 


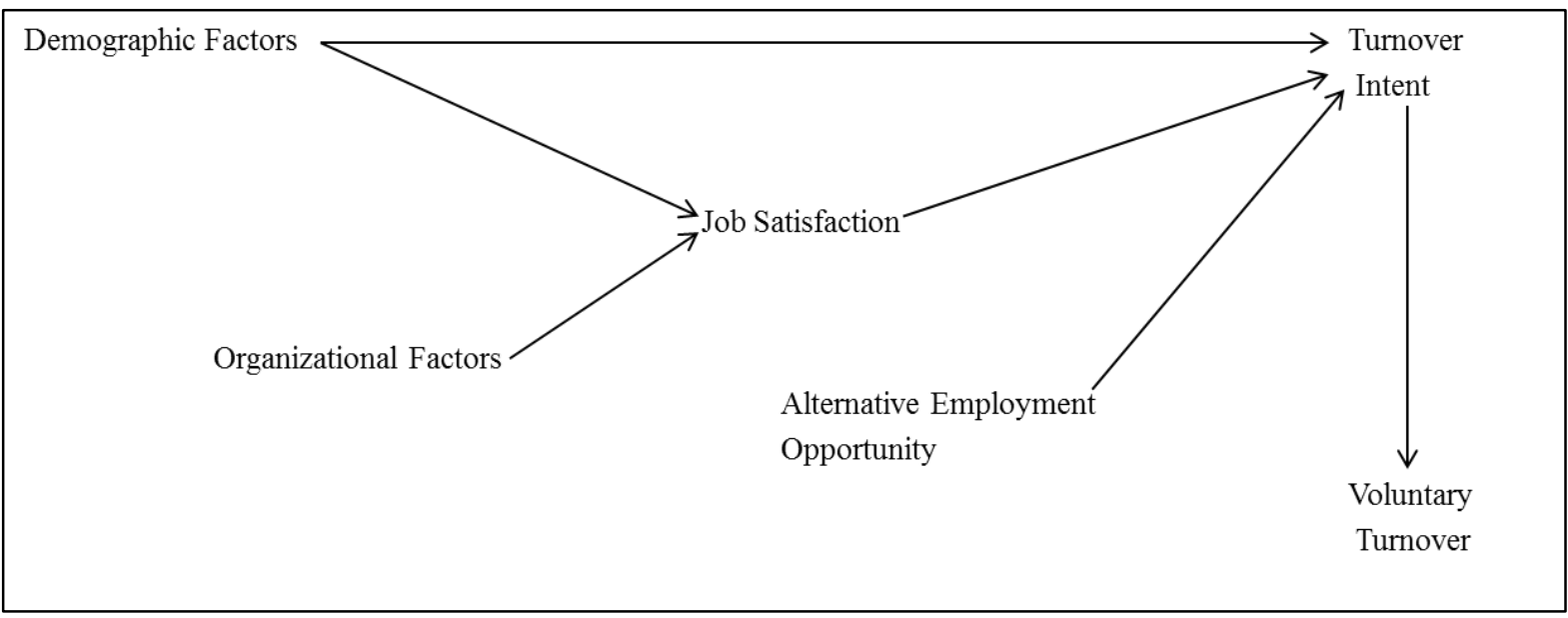

Figure 2. Conceptual model of turnover proposed by Lambert, Hogan, \& Barton (2001). 


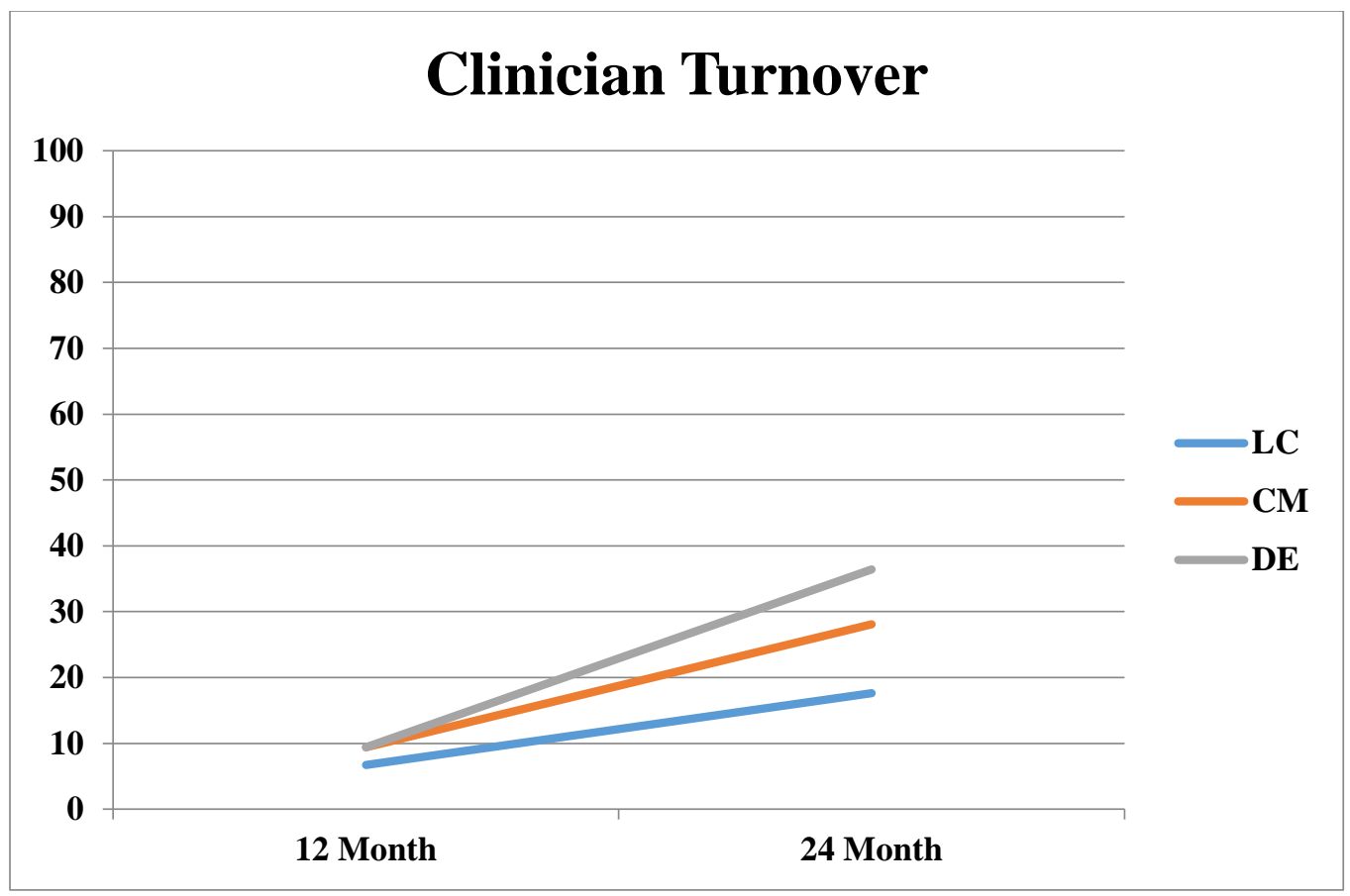

Figure 3. 12- and 24- month rates of clinician turnover by training condition LC - Learning Collaborative; CM - Cascading Model; DE - Distance Education 


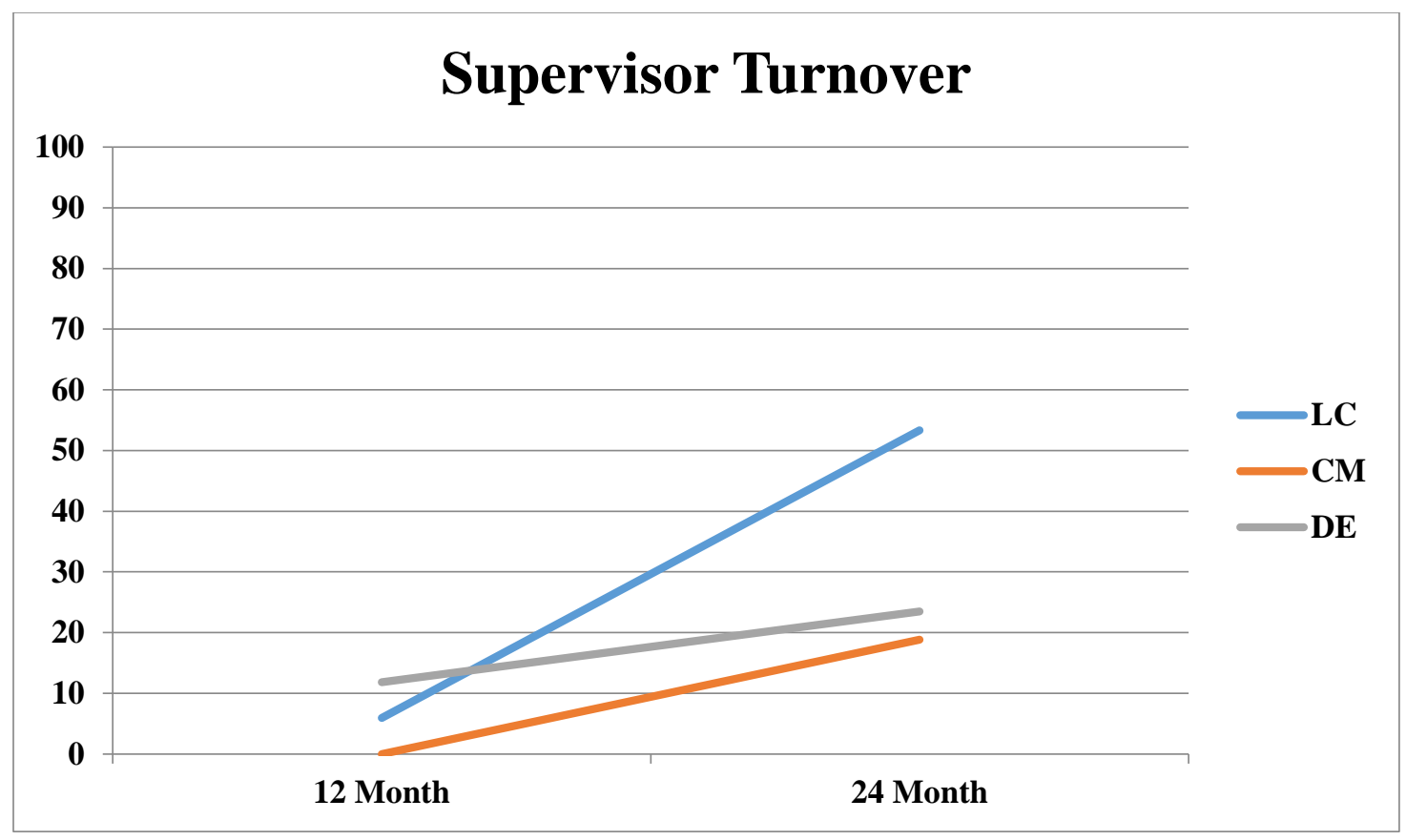

Figure 4. 12- and 24- month rates of supervisor turnover by training condition. LC - Learning Collaborative; CM - Cascading Model; DE - Distance Education 


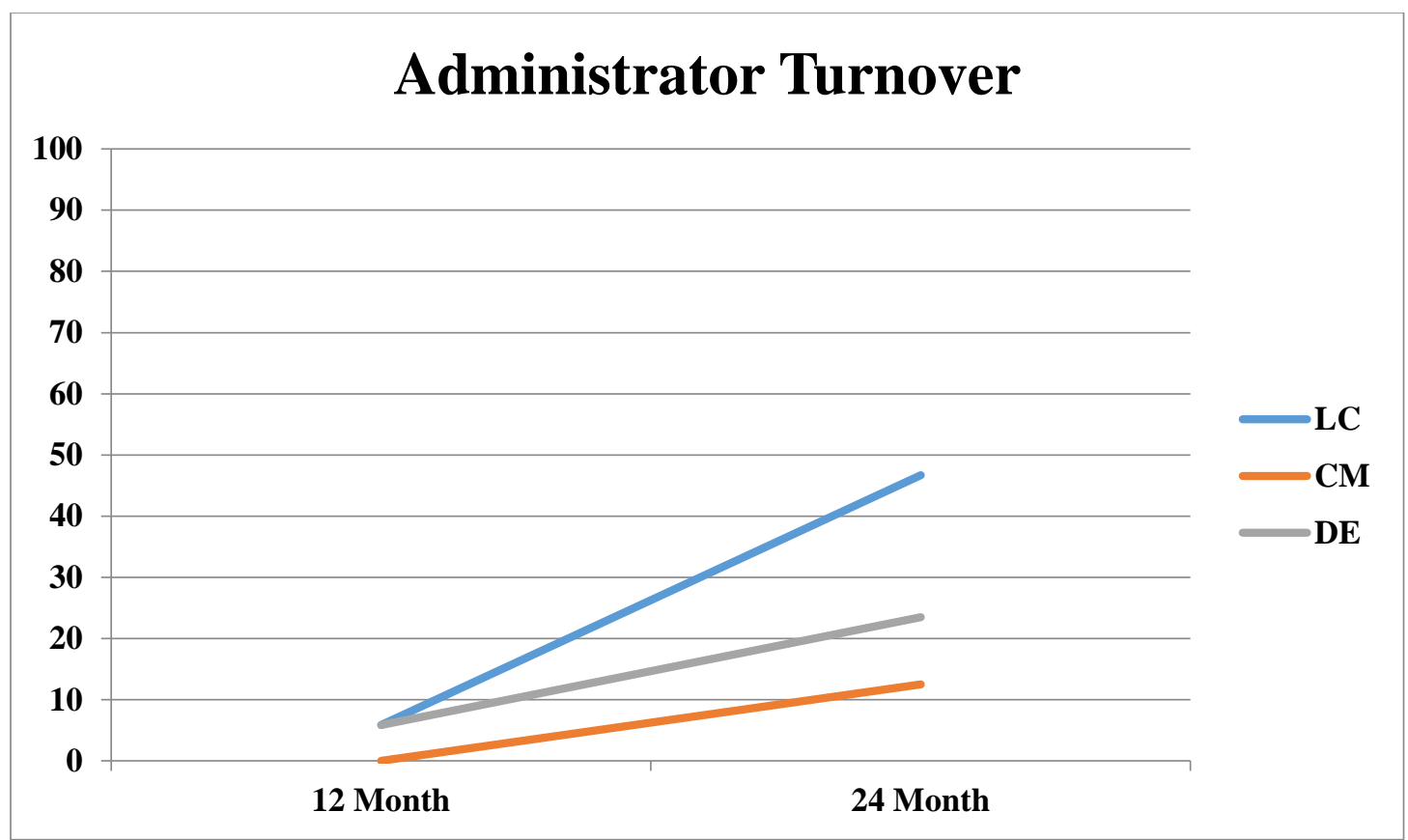

Figure 5. 12- and 24- month rates of administrator turnover by training condition. LC - Learning Collaborative; CM - Cascading Model; DE - Distance Education 


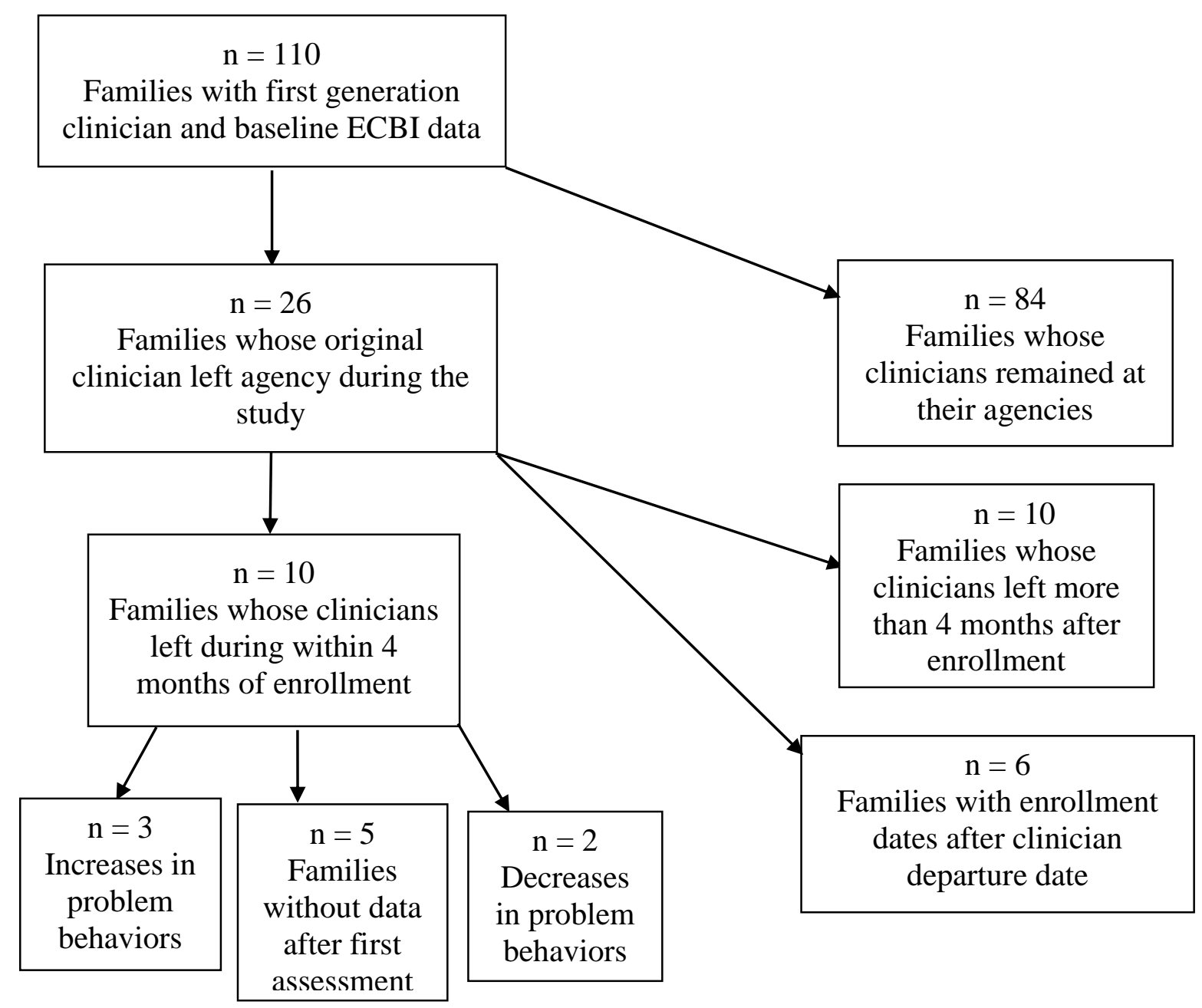

Figure 6. Flow diagram depicting families who may have experienced service disruption as a result of clinician turnover. 


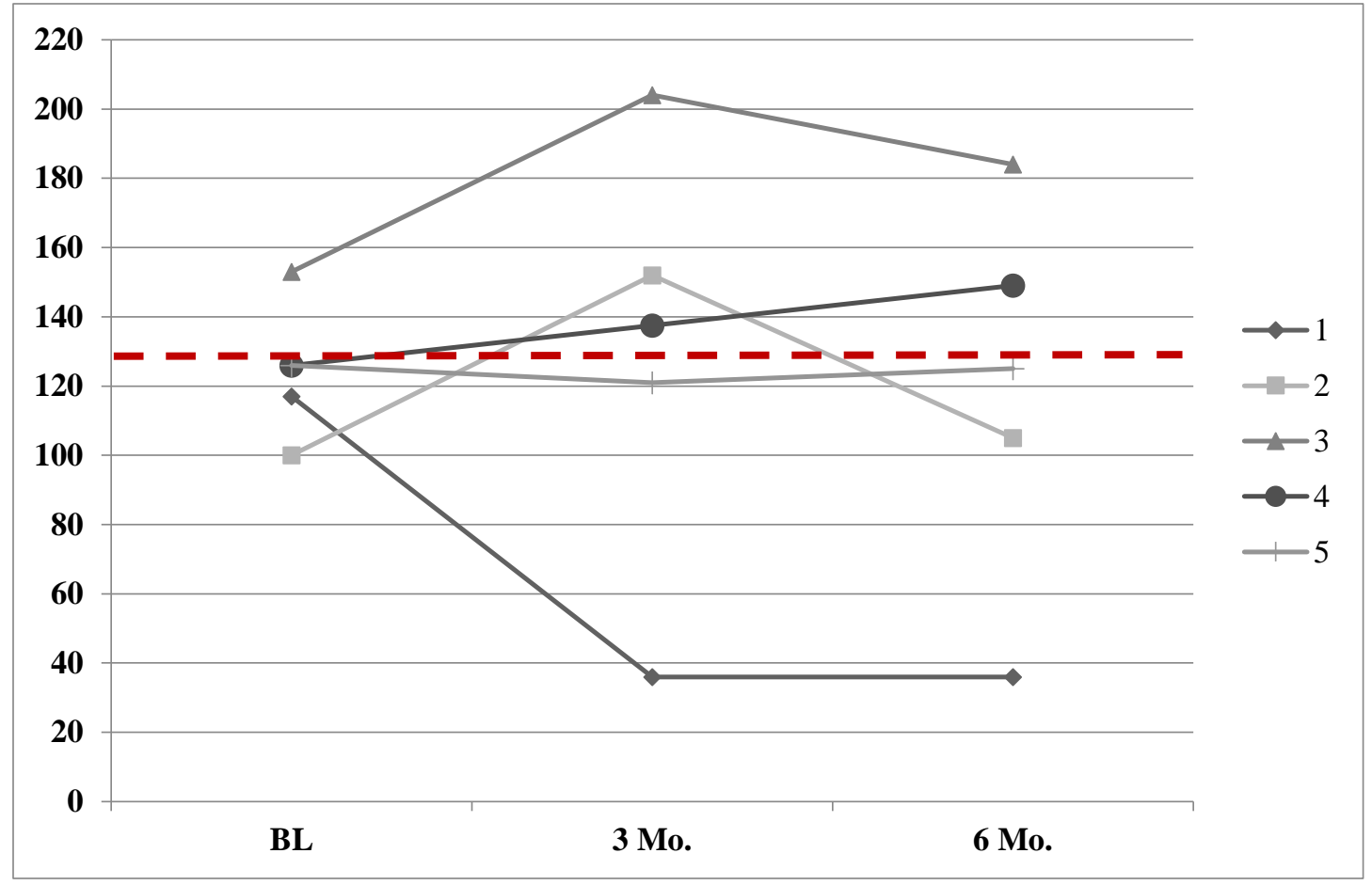

Figure 7. ECBI Intensity scores over time for the five families whose clinicians left during their treatment phase and for whom data were available. Note: red line indicates the clinical cutoff score. 


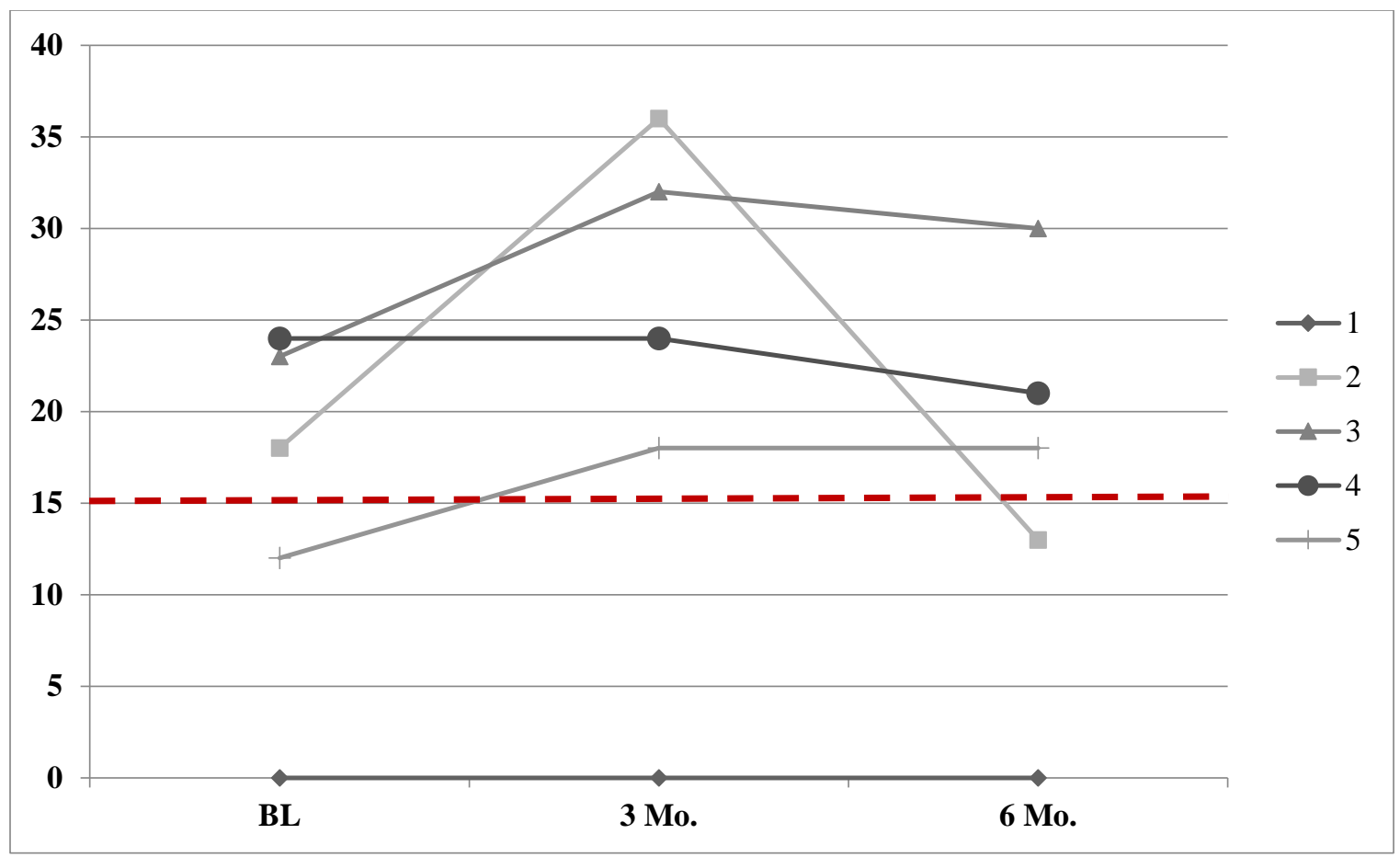

Figure 8. ECBI Problems scores over time for the five families whose clinicians left during their treatment phase and for whom data were available. Note: red line indicates the clinical cutoff score. 
Appendix

\section{Family Information}

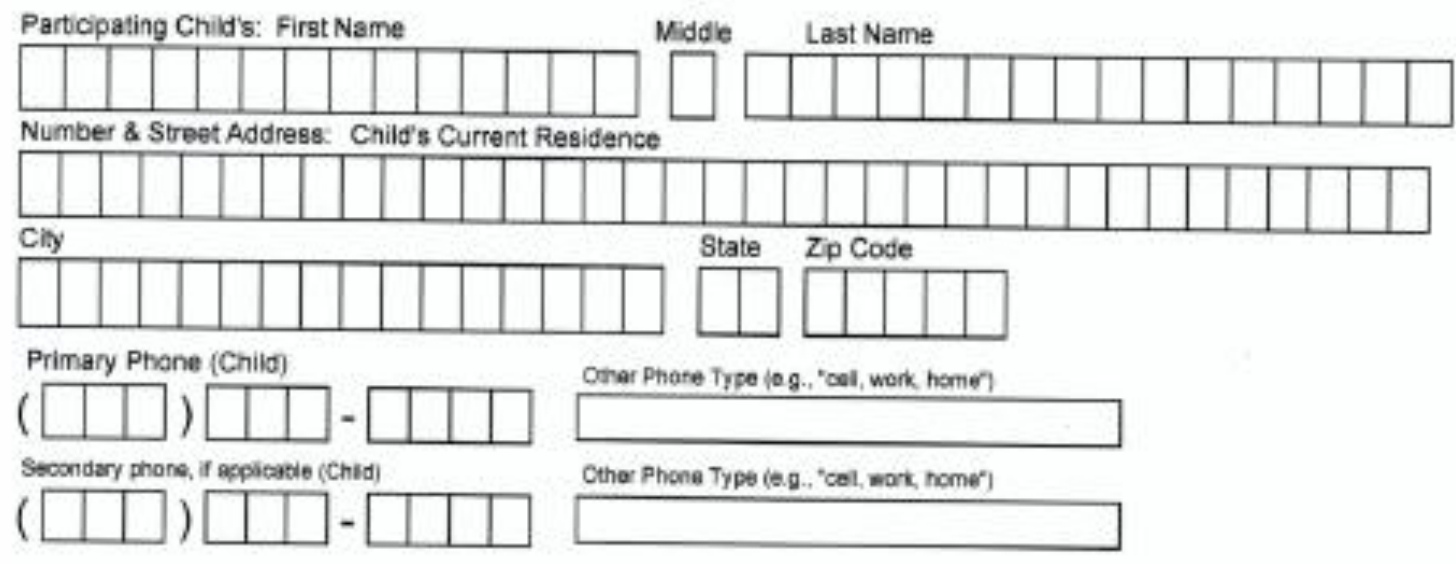

Date I am filling out this form:

Adult filling out this form:

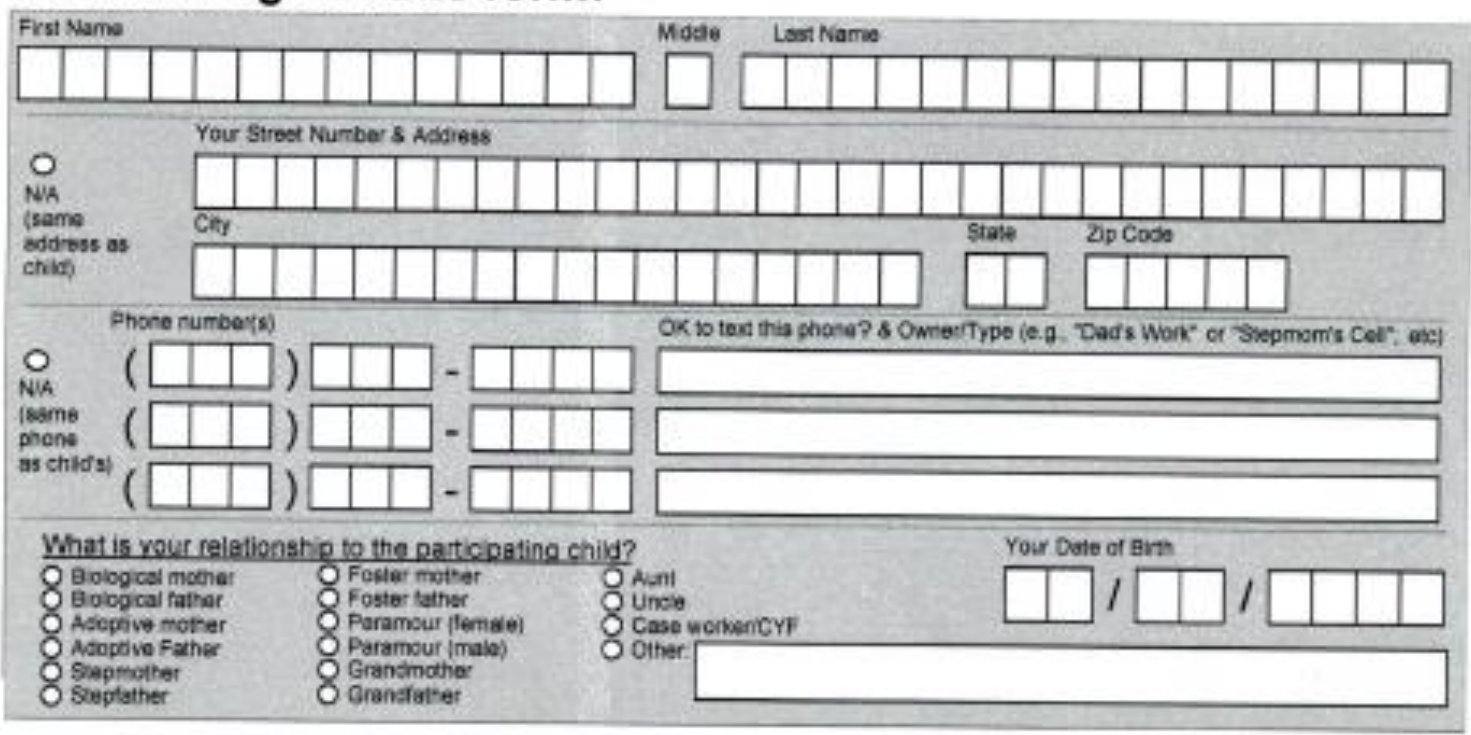




\section{Other Parent(s)/Guardian(s)/Adult(s) in child's household or involved with child's care:}
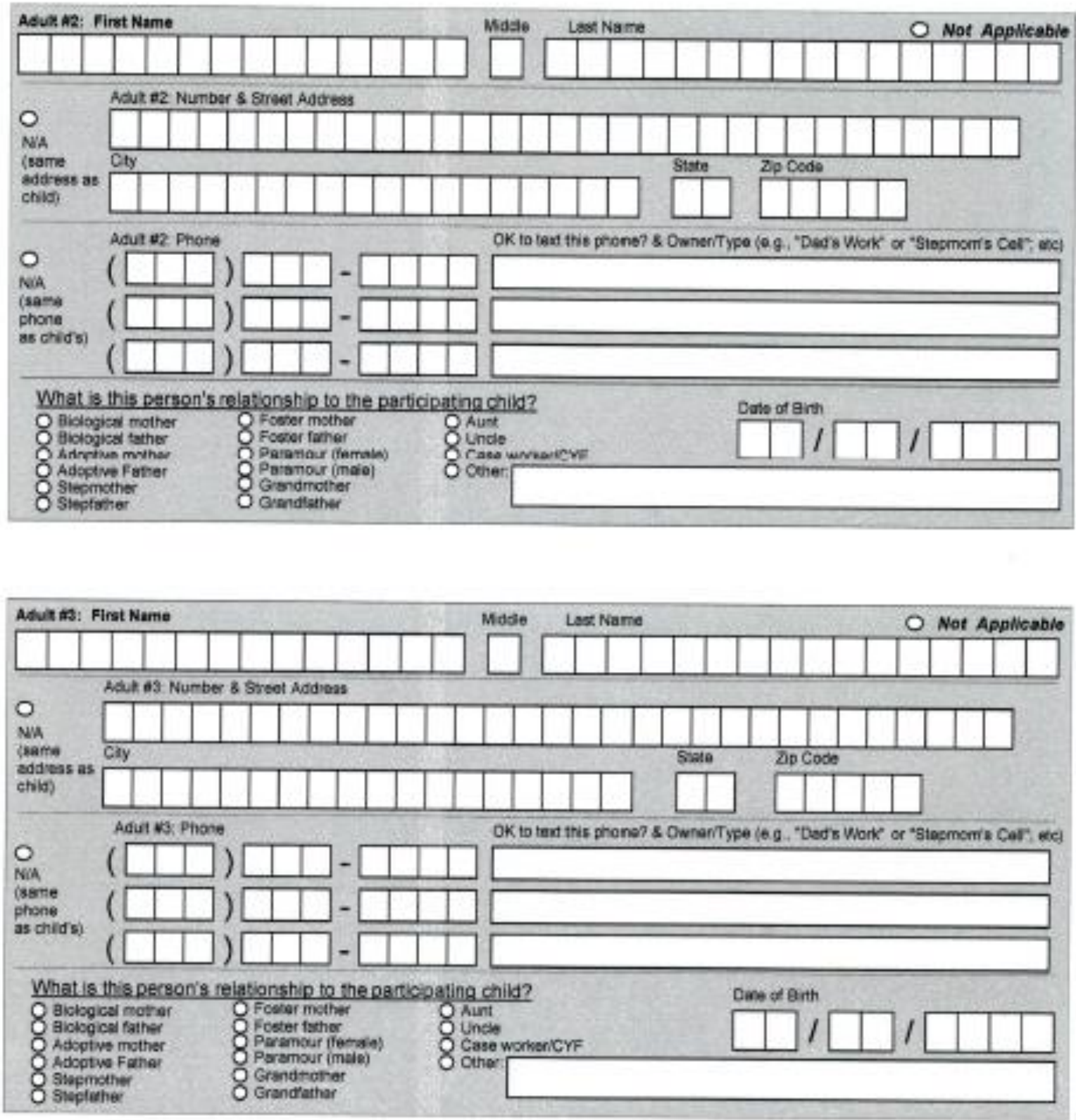


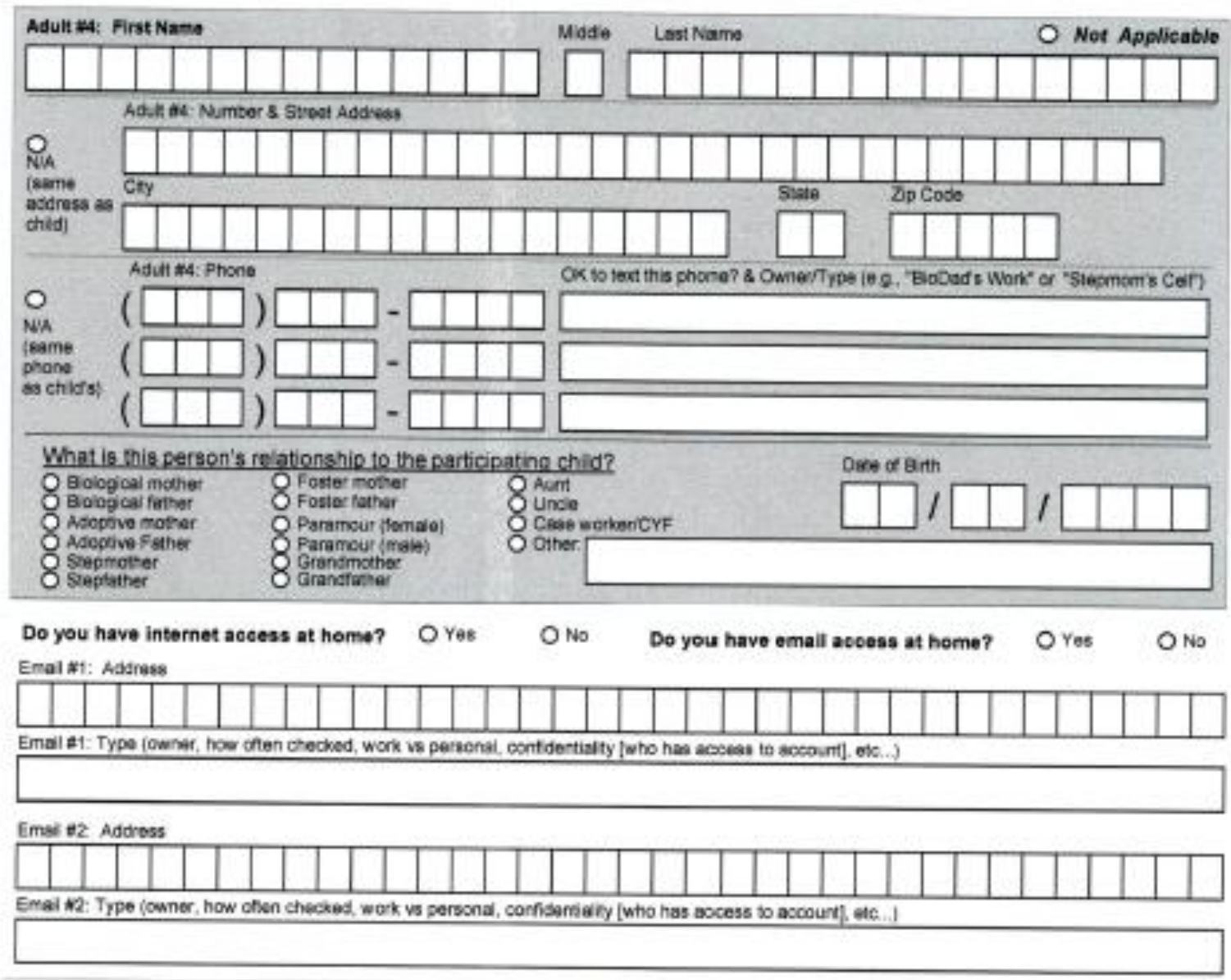

\section{Placement / Residential Changes}

Has this child lived in the same place throughout the past year for sinca the last assessment)? $O$ Yes O No If Yes, SKIP to the next page.

Is this child curently in a placement ananged by someane culside of the tamily? O Yes O No

If Yos, where is the child currently placed, and how long has heishe been there?

What was the reason for the placement?

Amy additional comments about placament?

If ehild is Not Currenty in placement, has heishe been in a placement arranged by someane outside of the famly (e.g., child welfare sysiem, court, otc...) at any time in the past year (or since the last assessment)?

If Yos, whare was the child placed, and how long was helshe thare?

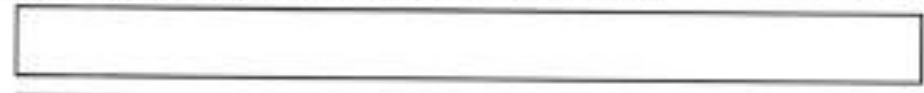

What was the reason for the placement?

Any addional comments about placement?
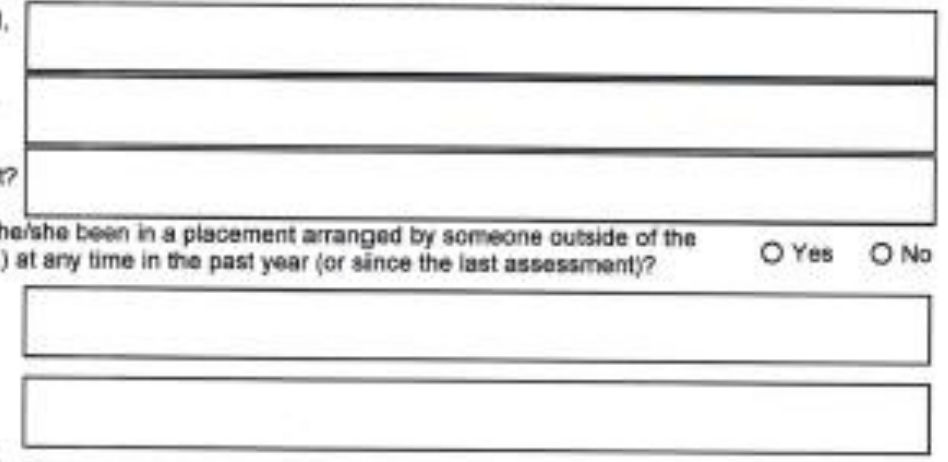


\section{Socio-economics, Demographics, Household Data}

\section{Is your household the same as child's household?}

$O$ Yes, ny househoid information is the same as chilfs household intormatian

If yes, How many months in the past year (or since last assessmenti has this child lived in the same heusehold as you?

O No, but still able to report at least some data on childs household

O No, unable to report any data an chid's househoid (SKIP THE REST OF THIS FAMILY INFO QUESTIONNAIRE)

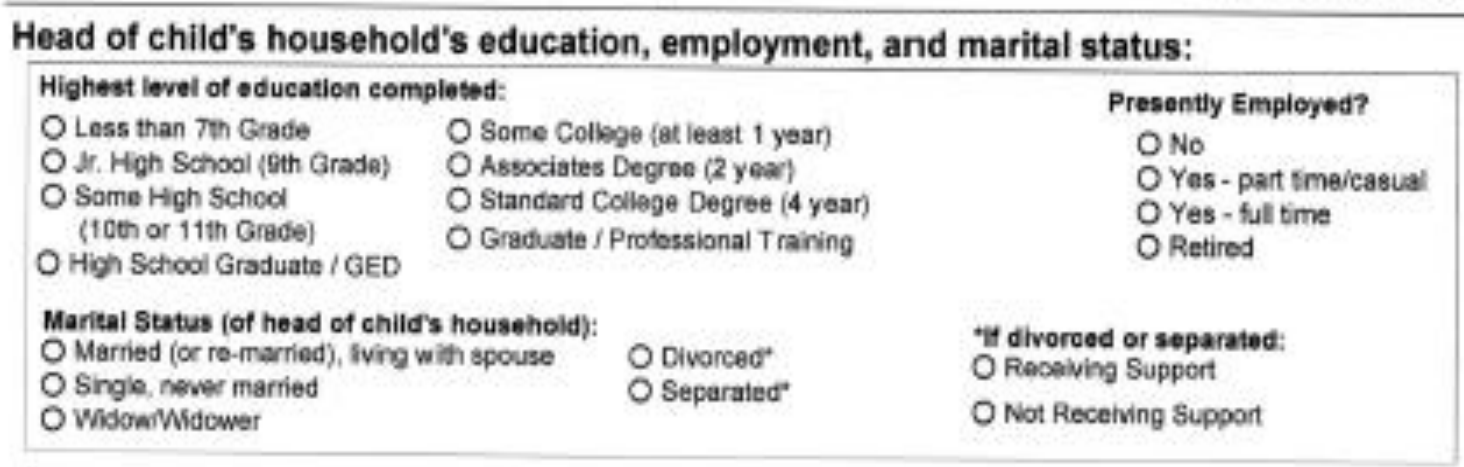

\section{Other parent/adult in child's household education and employment status: 0 Not Applicable}

$\begin{array}{ll}\text { Highest level of edueation sompleied. } \\ \text { O Less than } 7 \text { th Grade } & \text { O Some College (at least } 1 \text { year) } \\ \text { O Ji. High School (gth Grade) } & \text { O Associates Degree (2 year) } \\ \text { O Some High School } & \text { O Standard Cologe Degree (4 year) } \\ \text { (10th or 11th Grade) } & \text { O Graduate / Profesaional Training } \\ \text { O High School Graduate I GED } & \end{array}$

Prosently Employed:

O No

O Yes - part time

O Yes - full time

O Retired

\# of adults in child's current home

Fof chidren currenty in the hame ( $<18 \mathrm{yr}$.)

Household income: Which of the following ranges best describes the (childs) heusehold income?

(include child support payments, disability payments, and all other sources of irevenue)
O $\$ 14,999$ or less
$0 \$ 15.000$ to $\$ 29.900$
O $\$ 30.000$ 10 $\$ 49,990$
$0 \$ 50,000$ \$ $\$ 74,989$
O \$75,000 or mare

Has the childifamily recelved any of the following services in the past year (or sinee last asessment)
A. Modicaid
B. CHIP
O Yes ONo
C. Food stamps
OYes ONO
E. Social Securlty Disability income
F. Sociel Securly Suppiemental Income
OYes ONo
D. WIC (Women with intants and Chidren) O Yos O No
G. Medicare (income eligbility-only, not age) $O$ Yes ONo
H. Amy ather type of social essistance?

OYes ONo

Does partielpating child have health insuranee currently O Yes O No (end of questionnaire)

(This includes Medicaid, CHIP, or cther public programs)

If yes, is this hoalth insuranes... (ehoose one)

O Private insurance, from empleyer or private healith plan $O$ CHIP

Medicaid

Other, specify:

In the past 12 months has there been a sme when the participating chlid has had no health insurance? $O$ Yes O No 


\section{Parent/Guardian/Adult Information}

Your Gender:
Male O Female

\author{
Your Ethnicity; \\ O Hispanic or Latino \\ O Not Hispanic of Latino \\ OUnknown \\ Your Race: \\ (Fill in all that apply) \\ O American Indian/Alaska native \\ O Asian \\ O Native Hawailan or Pecific Islander \\ O Black or African American \\ o White \\ o Unknown
}

Please Answer BOTH the Ethnicity AND Race fields

\section{Child Information}

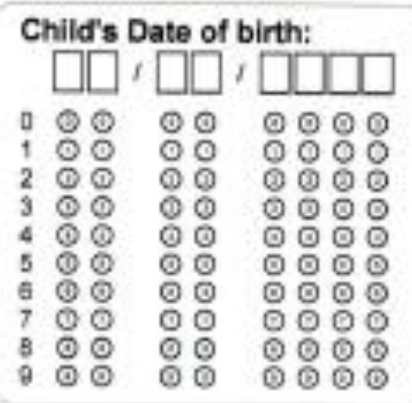

Child's Gender:

O Male

O Female

\section{Child's Ethnicity:}

O Hispanic or Latino

O Not Hispanic or Latino

OUnknown

Child's Race:

(Fill in all that apply)

O American Indian/Alaska native

OAsian

O Native Hawailan or Pacific Islander

O Black or African American

White

O Unknown
Please Answer BOTH the Ethnicity AND Race fieids. 
Administrator Background Form ${ }^{\text {Participant ID: }}$

Please answer the following questions about your demographic information, your professional background, and your current job.

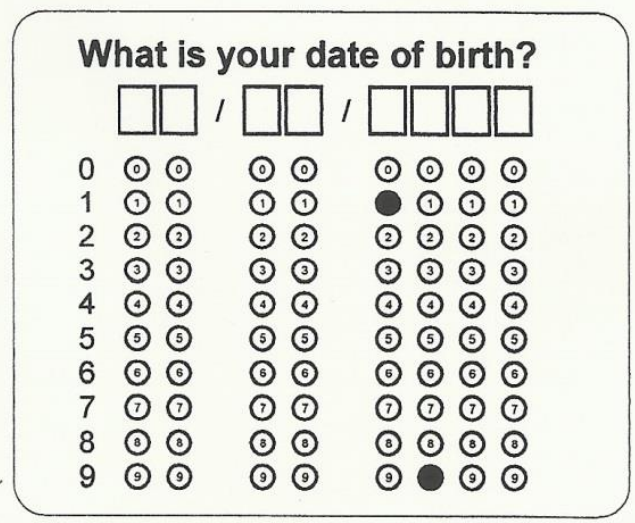

What is your Gender?

O Male

O Female

What is your Ethnicity?

O Hispanic or Latino

O Not Hispanic or Latino

What is your Race?

(Fill in all that apply)

O American Indian/Alaska Native

O Asian

O Native Hawaiian or other Pacific Islander

O Black or African American

OWhite

O More than one Race
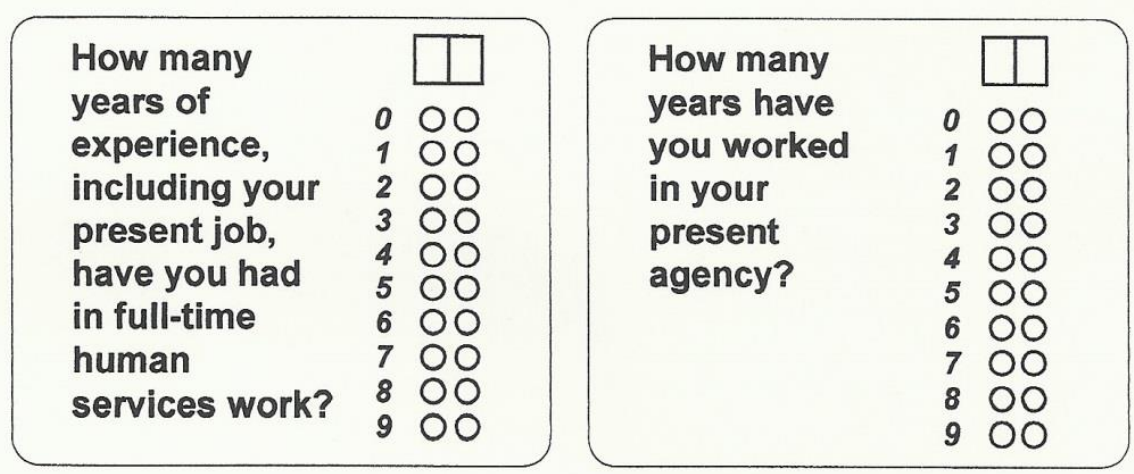

Your highest level degree is in:

What level of education
have you completed?

O High school graduate

O Some college

O Bachelors degree

O Some graduate work

O Masters degree

O Doctoral degree

O Education

O Medicine

O Nursing

O Psychology

O Social Work

O Other
Please Answer BOTH the

Ethnicity AND Race fields.

What is your current role? (Fill in all that apply)

O Behavioral Health Director O Chief Executive Officer (CEO)

O Chief Financial Officer (CFO) O Children's Mental Health Director

O Clinical Director O Executive Director

O President O Program Director

O Vice President O Other, please specify: 


\section{Administrator Contact Information Forticipant 10 \\ Administrator Contact Information Form}

\section{Please list your current Agency, Work Contact Information, Home Contact Information and Internet Acess / Email}

Name of agency where you will be providing PCIT:

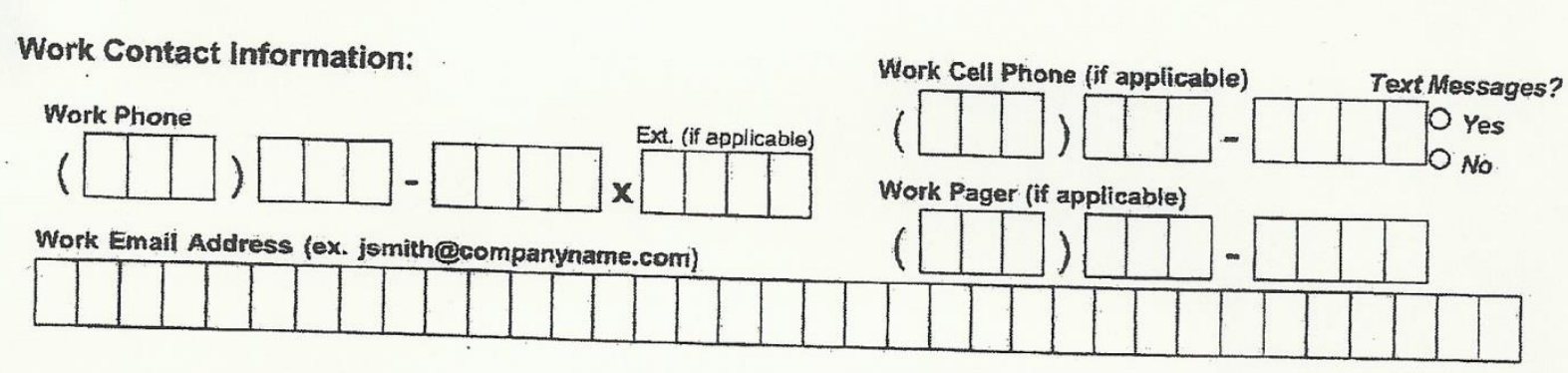

\section{Home Contact Information:}

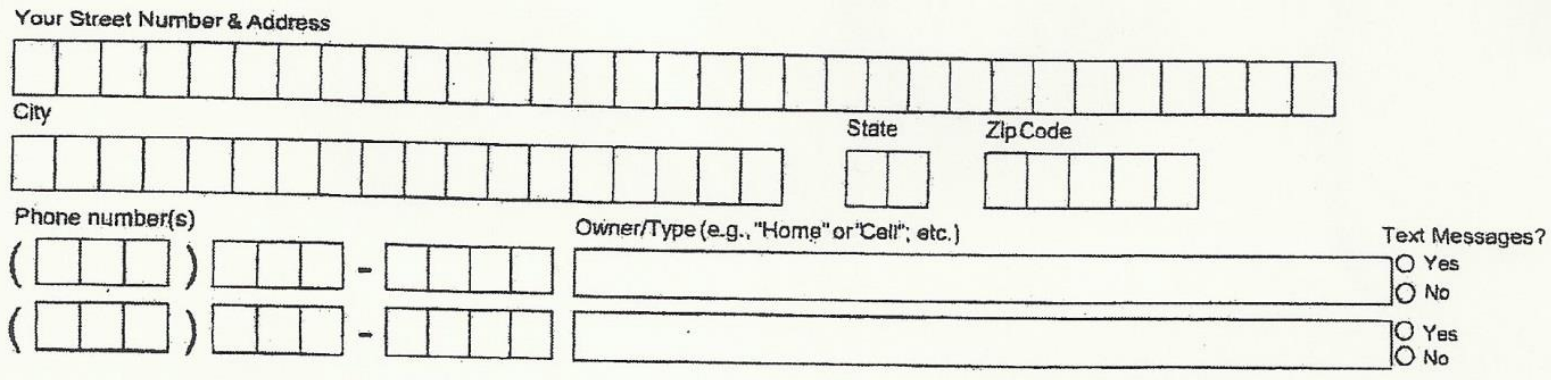


Participant ID:

\section{Internet Access / Email:}

Do you have internet access at home? O Yes O No Do you have email access at home? $\mathrm{Y}$ Yes NoO

Email\#1: Address

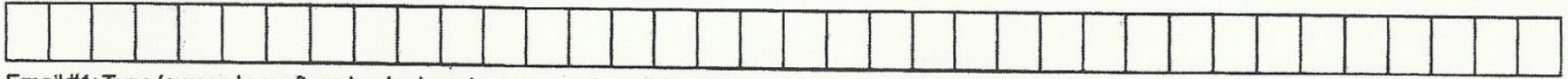

Email\#1:Type (owner, how often checked, work vs personal, confidentiality [who has access to account], etc...)

(access to account], etc...)

Email \#2: Address

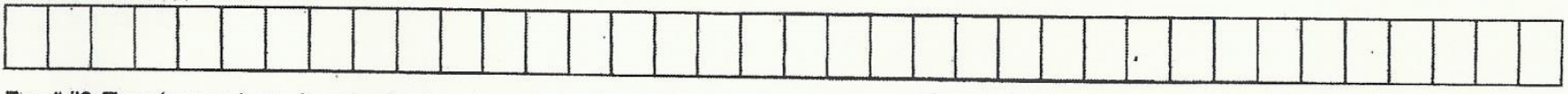

Email \#2: Type (owner, how often checked, work vs personal, confidentiality [who has access to account], etc...)

What is your preferred method of communication?

(Please check one)

O Email O Work Phone O Home Phone O Cell Phone O Text

Please provide information for another person who may help us contact you.

Person's Name (first)

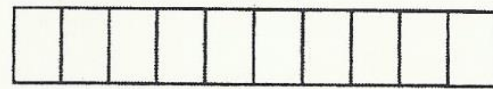

(last)

Number \& Street Address

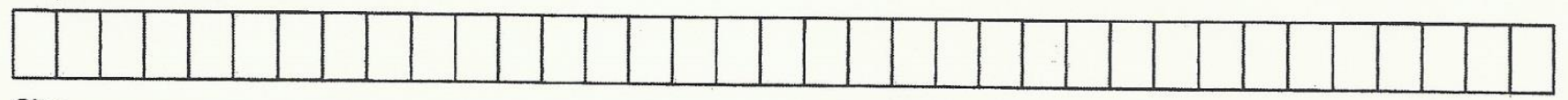

City

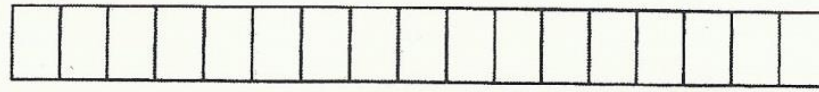

Phone

State

Zip Code
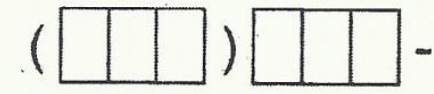

This person's relationship to you: 


\section{B. Eyberg Child}

\section{Parent Rating Form by Sheila Eyberg, PhD}

Your Name Relationship to Child Today's Date

Child's Name Child's Gender Child's Date of Birth

Directions: Below are a series of phrases that describe children's behavior. Please (1) circle the number describing how often the behavior currently occurs with your child, and (2) circle either "yes" or "no" to indicate whether the behavior is currently a problem for you.

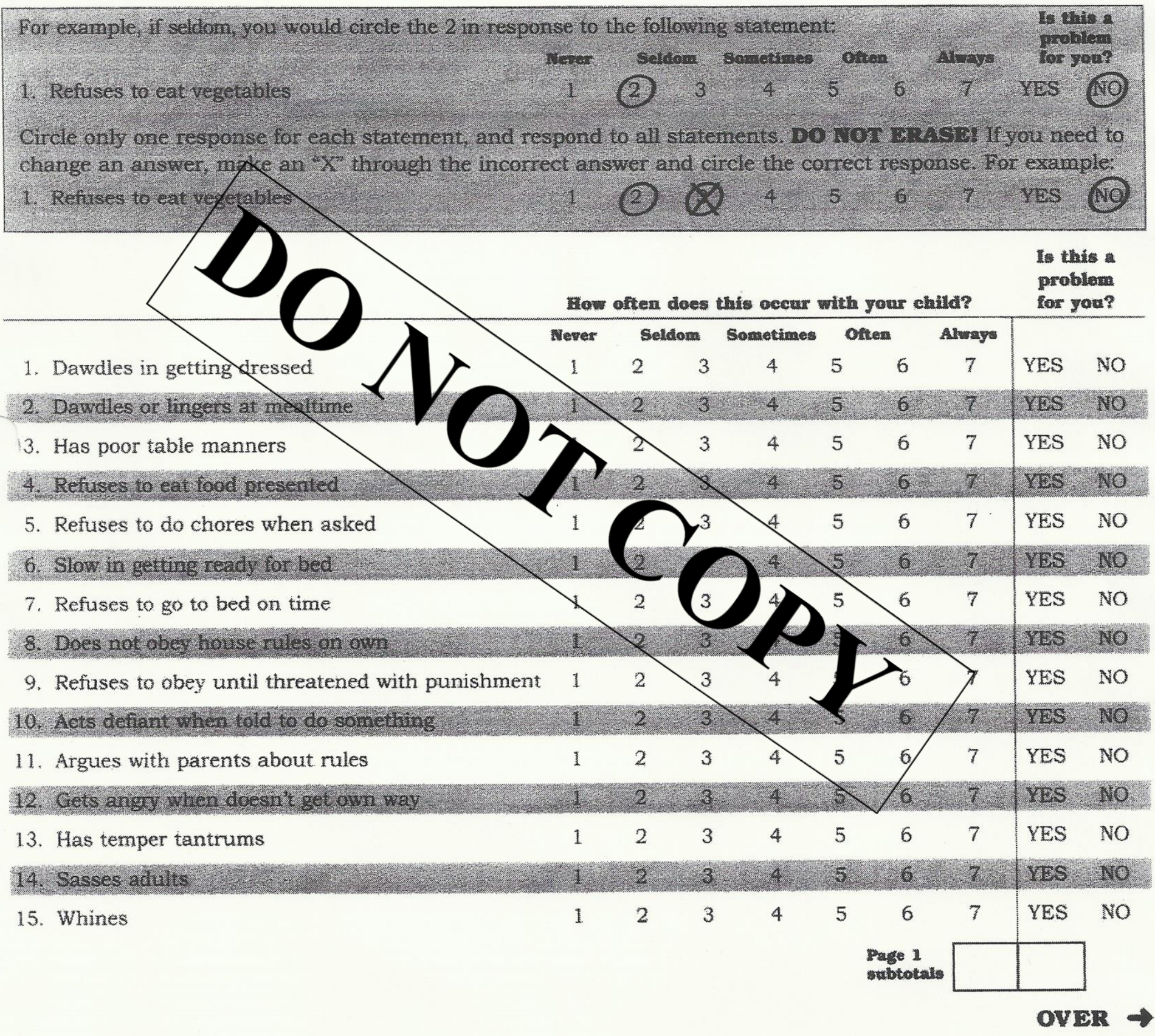

14R. 16204 N. Florida Ave. - Lutz, FL 33549 - 1.800.331.8378 - www.parinc.com

Copyright 1998,1999 by PAR. All rights reserved. May not be reproduced in whole or in part in any lorm or by any means without written permission of PAR. This form is printed in burgundy ink on white paper. Any other version is unauthorized. 


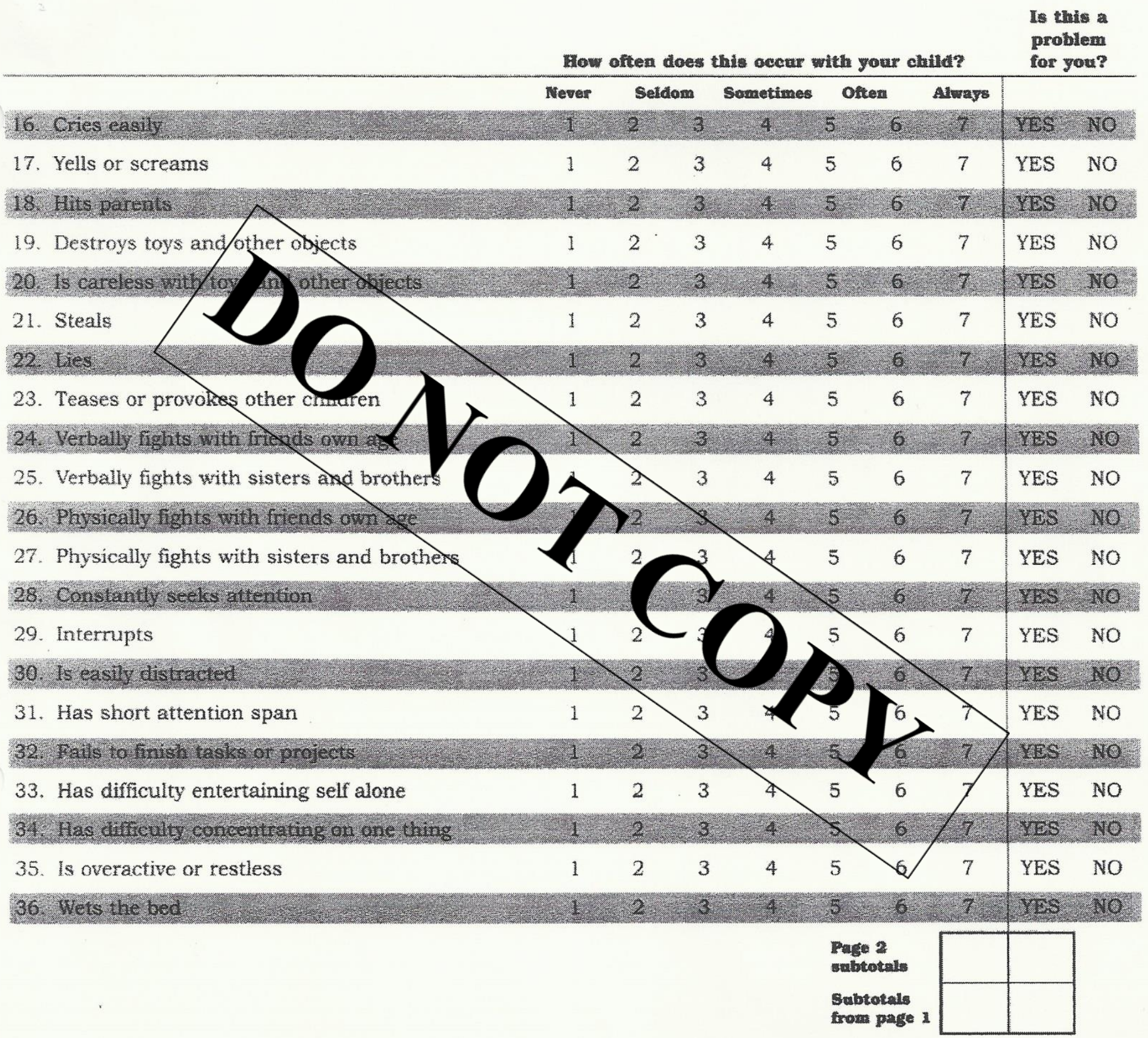

\begin{tabular}{|l|l|l|l|}
\hline \multicolumn{1}{|c|}{ Scores } & Raw score & T score & $\begin{array}{c}\text { Exceeds Cutofi } \\
(\mathrm{V})\end{array}$ \\
\hline Intensity & & & \\
\hline Problem & & & \\
\hline
\end{tabular}

\section{Comments:}




\section{Clinician Background and Contact Information Form}

Please answer the following questions about your demographic information, your professional background, and your current job.

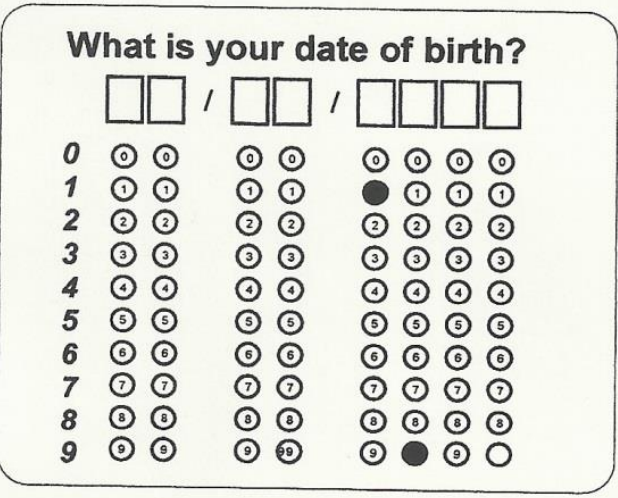

What is your Gender?

O Male

O Female

What is your Ethnicity?

O Hispanic or Latino

O Not Hispanic or Latino

What is your Race?

(Fill in all that apply)

O American Indian/Alaska Native

O Asian

O Native Hawaiian or other Pacific

Islander

O Black or African American

OWhite

O More than one Race

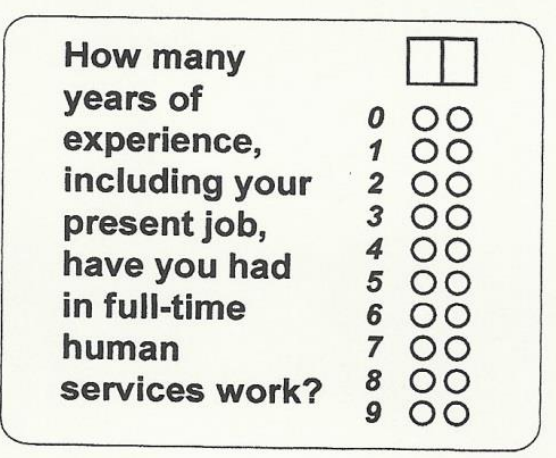

\begin{tabular}{|lc|}
\hline How many & $\square$ \\
years have & 000 \\
you worked & 100 \\
in your & 200 \\
present & 300 \\
agency? & 400 \\
& 500 \\
& 600 \\
& 700 \\
& 800 \\
& 900 \\
\hline
\end{tabular}

Your highest level degree is in:

What level of education
have you completed?

O High school graduate

O Some college

O Bachelors degree

O Some graduate work

O Masters degree

O Doctoral degree

Please Answer BOTH the

Ethnicity AND Race fields.
O Education

O Medicine

O Nursing

O Psychology

O Social Work

O Other

What is your current role? (Fill in all that apply)

OAssessment Clinician O Supervisor

O Intake Coordinator

O Treatment Clinician

O Program Support

O Other, please specify: 


\section{Are you professionally licensed (e.g., psychology or social work license?) O Yes O No Please indicate type of license: O LPC O LSW O LCSW O Psychologist O Other, please specify:}

2. Setting where you do the majority of your work:

O Client's homes

O Your agency

O Group home/residential

Other, please specify:

\section{Please rate your level of experience in the following areas:}

For each of the following items, please fill-in one number to indicate your experience. Use the following scale:

\section{None (Novice)}

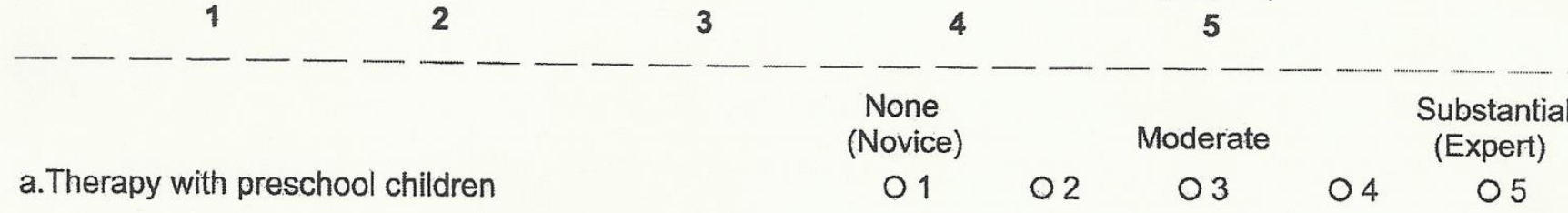

\section{Moderate}

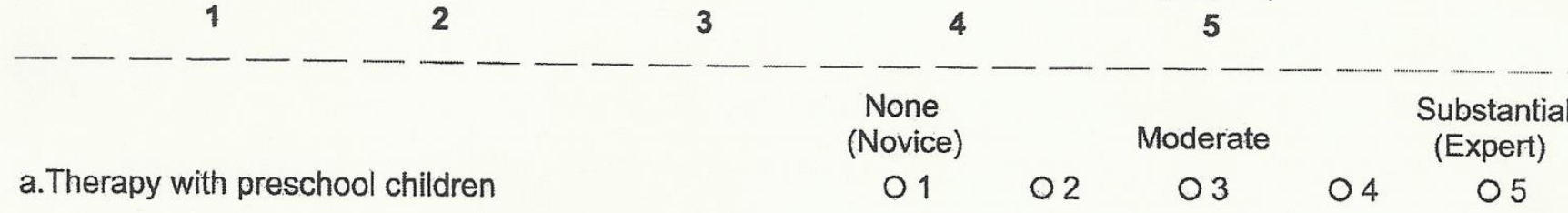

Substantial

(Expert)
b. Assessment of child and family functioning
01
02
03
04
05

c. Cognitive Behavioral Therapy

01

02

03

04

05

d. Family Therapy

$01 \quad 02$

03

04

05

e. Behavioral Parent Training

02

03

04

05

4. In the last 6 months, what trainings have you attended? Please list the topics of any trainings here:

a.

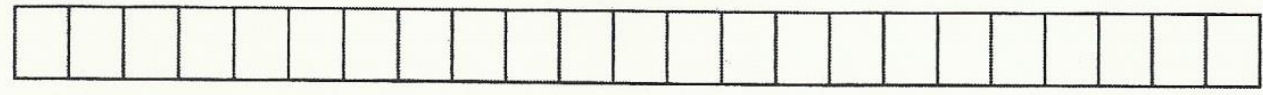

b.

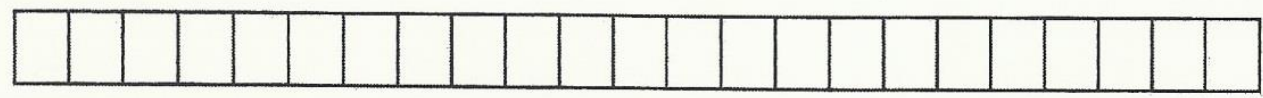

c.

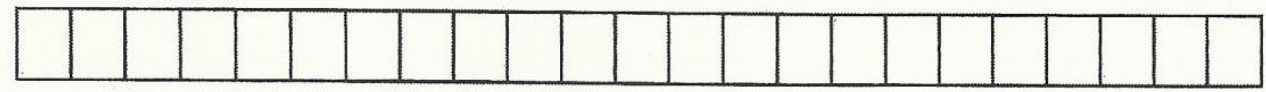

8. How long was each training (in hours)?

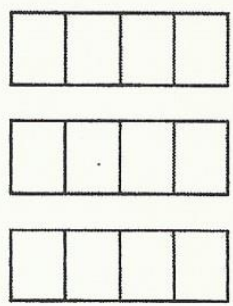

(hours) 
Please list your current Agency, Agency Program(s), Supervisor(s), Work Contact Information, ome Contact Information and Internet Access / Email

Agency where you will be providing PCIT:

O Devereux Community Services

O Holcomb Behavioral Health Systems

O Child Guidance Resource Centers
O University Community Behavioral Health

O Alternative Community Resource Program

O Nulton Diagnostic and Treatment Center

\section{Agency Program(s):}

Primary Agency Program that you work in (Primary agency program should be the one that you do most of your work in) OBehavioral Health Rehabilitation Services / Wraparound O Family-Based O Inpatient O Outpatient

O Partial Hospitilization O School-Based O Other, please specify:

N/A Secondary Agency Program that you work in (Bubble "N/A" if you work in only one agency program)

OBehavioral Health Rehabilitation Services / Wraparound O Family-Based O Inpatient O Outpatient

O Partial Hospitilization O School-Based O Other, please specify:

Clinical Supervisor(s): (Primary supervisor should be the direct supervisor with whom you have the most contact)

Primary Supervisor's Name (first)

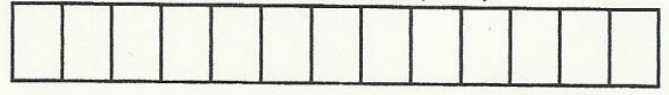

Secondary Supervisor's Name (first) (last)

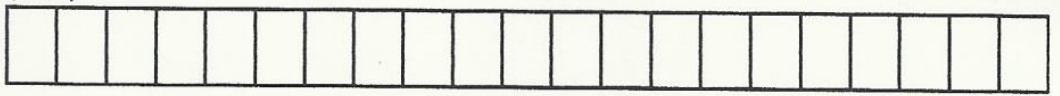

(last) (Bubble "N/A" if you only have 1 direct supenvisor.)

$\circlearrowleft$
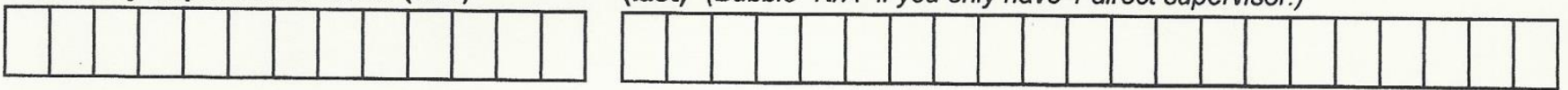

Work Contact Information:

Work Cell Phone (if applicable)

Ext. (if applicable)

Work Phone
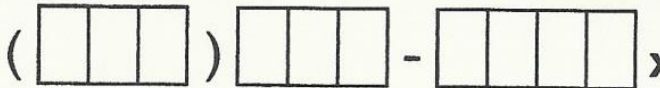

$\mathbf{x}$

Work Email Address (ex. jsmith@companyname.com)

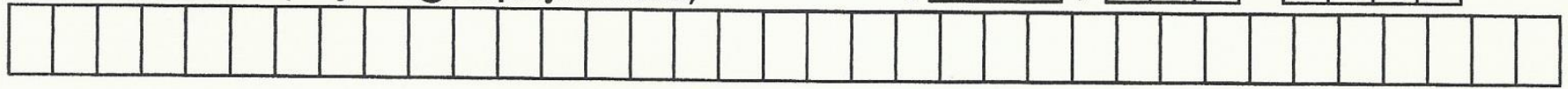




\section{Home Contact Information:}

Your Street Number \& Address

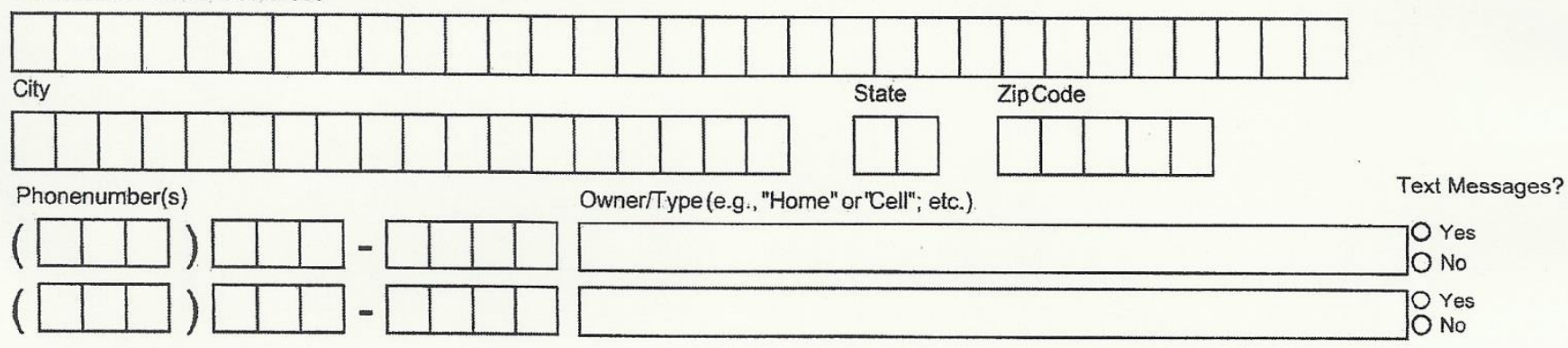

\section{Internet Access / Email:}

Do you have internet access at home? O Yes $O$ No Do you have email access at home? $\mathrm{Y}$ Yes NoO

Email\#1: Address

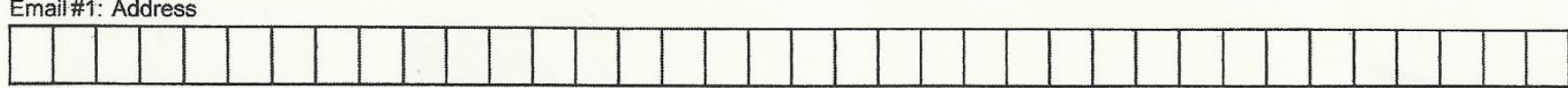

Email\#1: Type (owner, how often checked, work vs personal, confidentiality [who has access to account], etc....)

Email \#2: Address

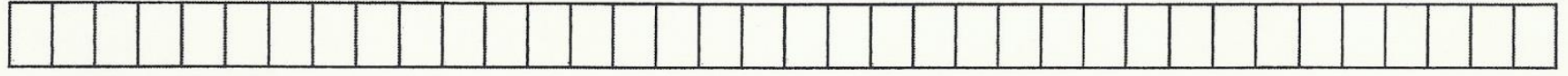

Email \#2: Type (owner, how often checked, work vs personal, confidentiality [who has access to account], etc...)

What is your preferred method of communication?

(Please check one)

Email O Work Phone $O$ Cell Phone O Home Phone O Text

Please provide information for another person who may help us contact you.

Person's Name (first)

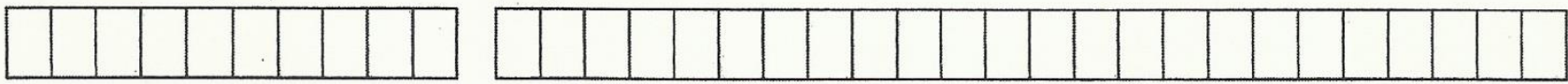

Number \& Street Address

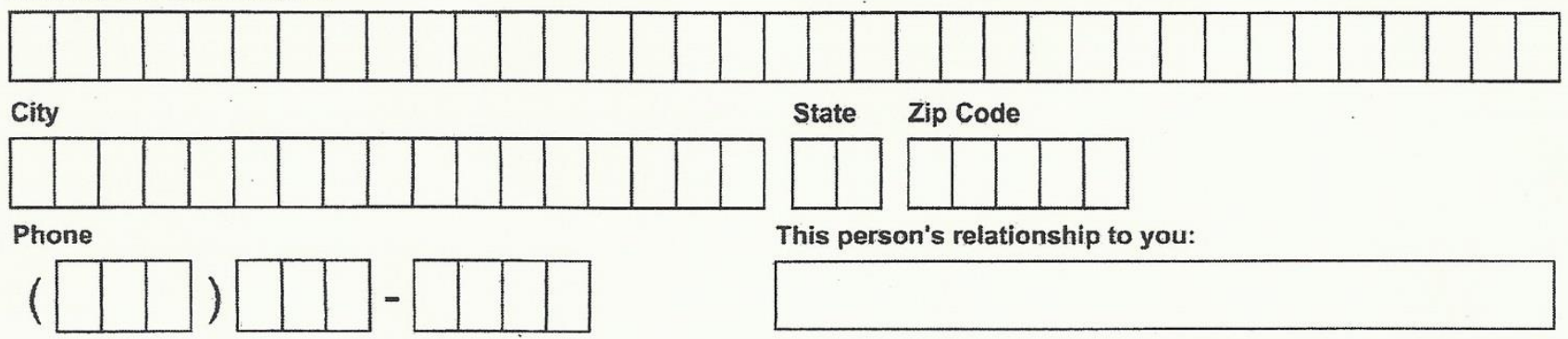


Participant ID:

\section{Clinician Background and Contact Information Form (continued)}

How many clients are you currently treating (i.e., total number on your caseload)?

Considering all the clients on your caseload, what percentage fall into the following age ranges: (These totals should add up to be $100 \%$ )

\begin{tabular}{|l|l|}
\hline & Percentage (\%) \\
\hline Under 3 years old & \\
\hline $3-5$ years old & \\
\hline $6-12$ years old & \\
\hline $13-18$ years old & \\
\hline Older than 18 years & \\
\hline
\end{tabular}

Considering all the clients on your caseload, what percentage of cases do you believe has had the following experiences? (some cases may have had multiple experiences, so the sum of all the percentages may exceed $100 \%$ )

\begin{tabular}{|l|l|}
\hline & Percentage (\%) \\
\hline Physical abuse/harsh physical discipline & \\
\hline Sexual abuse & \\
\hline Emotional abuse & \\
\hline Neglect & \\
\hline $\begin{array}{l}\text { Other child welfare concerns } \\
\text { please specify: }\end{array}$ & \\
\hline
\end{tabular}

Considering all the clients on your caseload, what percentage of cases do you believe has had the following diagnoses? (some cases may have had multiple diagnoses, so the sum of all the percentages may exceed $100 \%$ )

\begin{tabular}{|l|l|}
\hline & Percentage (\%) \\
\hline Developmental delays & \\
\hline Autism spectrum Disorder & \\
\hline $\begin{array}{l}\text { Internalizing Disorders (e.g., anxiety, } \\
\text { depression) }\end{array}$ & \\
\hline $\begin{array}{l}\text { Externalizing Disorders (e.g., disruptive } \\
\text { behavior disorders, ADHD, Conduct Disorder) }\end{array}$ & \\
\hline Learning Disorders & \\
\hline
\end{tabular}

What is the primary service area for your organization? (please check one)
$\square$ Rural
$\square$ Suburban
$\square$ Urban 
Does your organization primarily serve?

Please circle yes or no for each

\begin{tabular}{lll}
\hline Adults & Yes & No \\
\hline Young children & Yes & No \\
\hline Clients involved with child welfare & Yes & No \\
\hline Clients involved with criminal justice & Yes & No \\
\hline
\end{tabular}

Are you employed part-time or full-time at your agency? (please check one)

$\square$ Full-time

$\square$ Part-time

a. If full-time, what is your annual salary?

b. If part-time, what is the hourly rate you get paid? 


\section{Agency Staff Change Form - PCIT ACROSS PA \\ Self-Report Version \\ For Clinicians}

It is important for us to know about any changes in how and/or where you work. We are interested in any changes since the last time you completed an assessment for PCIT Across PA.

Have you been employed at the same agency since your last assessment?

IF NO,

What date did you leave your Agency/Organization?

What is the name of the new agency/organization you work for?

What type of agency/organization is it? (pick the one that best describes it)

- Private practice

- Community Mental Health Center

- Hospital System

- Other (please specify):

Is the new agency/organization in the PCIT Across PA study? Yes No

What program or service line with the new agency/organization are you now working in?

(pick all that apply)

- Outpatient

- BHRS

- Family-based

- Other (please specify):

What is your role in the new organization? (pick all that apply)

- Clinician

- Supervisor

- Program Manager

- Director

- Other (please specify):

Have you changed your program (service line) within your Agency/Organization?

If YES,

What date?

What is your new program (service line)? (pick all that apply)

- Outpatient

- BHRS

- Family-based

- Other (please specify):

Do you have a new role (job type) within your Agency/Organization?

IF YES,

What date did you change your role (job type?)

What is your new role (job type)? (pick all that apply)

- Clinician

- Supervisor 
- Program Manager

- Director

- Other (please specify):

Have you had a change in your clinical supervisor?

IF YES,

What date did you have a change in your clinical supervisor?

Is the new clinical supervisor participating in the PCIT Across PA study? Yes No

IF YES,

What is the name of your new supervisor?

Have you had a change in the number of hours your work (moving from part-time to full-time or vice versa)? Yes No

What date did your change in hours take effect?

Please describe the change in hours (pick one)

- Decrease in Hours

- Increase in Hours

Have you taken a leave of absence (e.g., maternity leave, FMLA, etc...) since your last assessment? Yes No

IF YES,

What date did your leave begin?

Please describe the reason for your leave:

Have you returned to work from a leave of absence (e.g., maternity leave, FMLA, etc...) since your last assessment? Yes No

IF YES,

What date did you return to work?

Please describe the reason for your leave: 


\title{
Survey of Organizational Functioning (TCU SOF)
}

\author{
Instruction Page
}

This survey asks questions about how you see yourself as a counselor and how you see your program.

To complete the forn pleas mark your answers by completely filling in the appropriate circles.

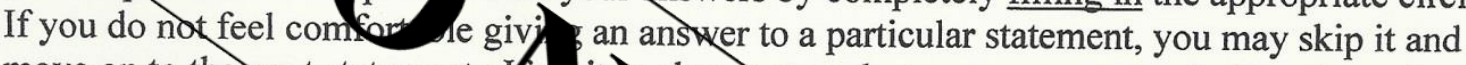
move on to the statement. blank. PLEASE DQNOT FOKD i RN The exanples below show how to mark the circles.

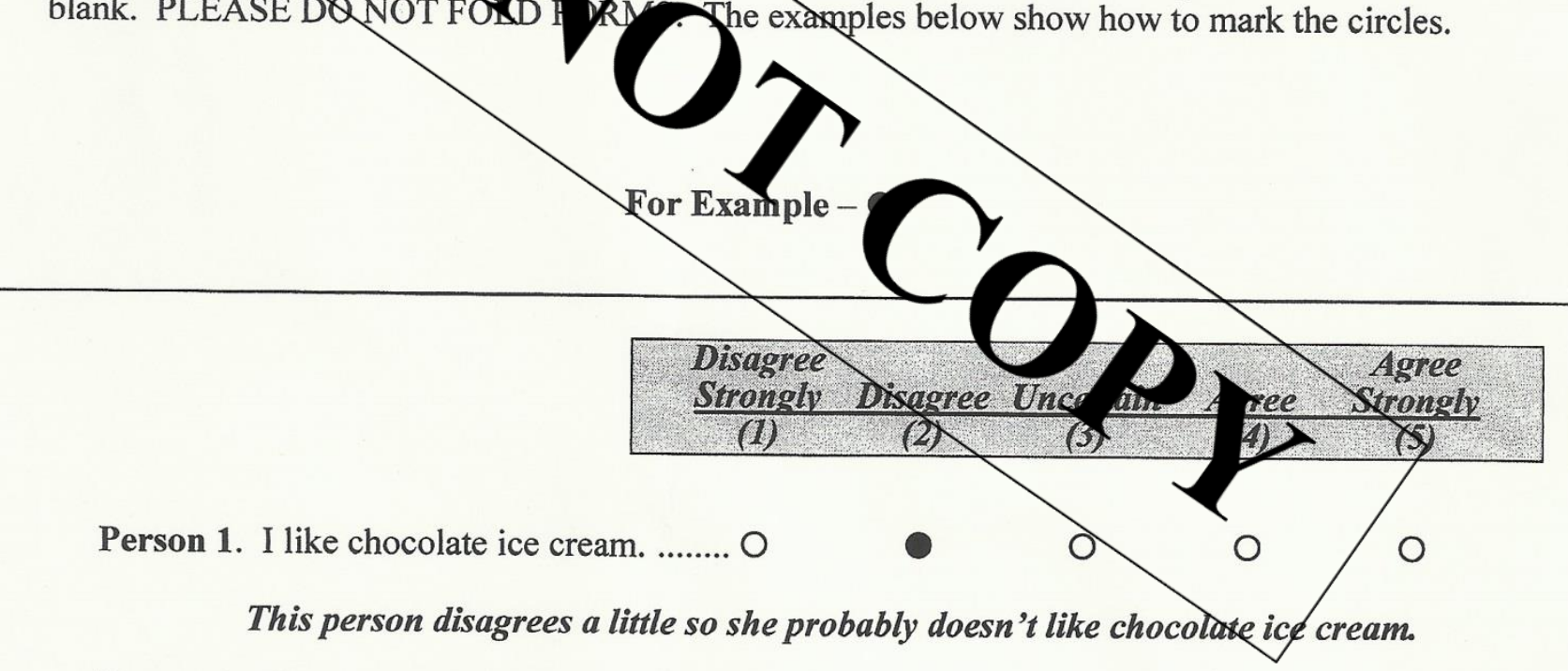

Person 2. I like chocolate ice cream. O

This person likes chocolate ice cream a lot. 


\section{Survey of Organizational Functioning (TCU SOF)}

PLEASE FILL IN THE CIRCLE THAT SHOWS YOUR ANSWER TO EACH ITEM.

\begin{tabular}{|ccccc|}
\hline $\begin{array}{l}\text { Disagree } \\
\text { Stronglv }\end{array}$ & Disagree & Uncertain & Agree & $\begin{array}{c}\text { Agree } \\
\text { Stronglv }\end{array}$ \\
\hline$(1)$ & $(2)$ & $(3)$ & $(4)$ & $(5)$ \\
\hline
\end{tabular}

Your program needs additional guidance in -

1. assessing client needs.

0

2. matching needs with services.

$\mathrm{O}$

3. increasing program participation by clients.

4. measuring client performance. O

5. developing $\mathrm{m}$ d effective outpatient

6.

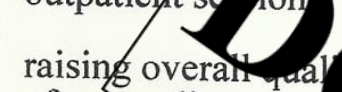
of courseling.

7. using client assessments to gujde clinical and program decisio.s.

8. using client assessments to documen program effectiveness.

You need more training for -

9. assessing client problems and needs.

10. increasing client participation in treatment. .. 0 O

11. monitoring client progress.

12. improving rapport with clients.

13. improving client thinking and problem solving skills.

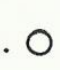

O 0

O

O 
Participant ID:

\begin{tabular}{lcccc}
$\begin{array}{l}\text { Disagree } \\
\text { Stronglv }\end{array}$ & Disagree Uncertain & Agree & $\begin{array}{c}\text { Agree } \\
\text { Stronglv }\end{array}$ \\
\hline (1) & (2) & (3) & (4) & (5)
\end{tabular}

Current pressures to make program changes come from -

17. clients in the program.

O

18. program staff members.

19. program supervisors or managers.

20. agency board ndembers

21. community

22.

23

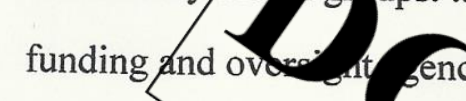

23. accreditation

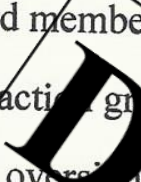<smiles>[CH]1[CH]C1</smiles><smiles>C1CCC(C2CCCCC2)CC1</smiles>
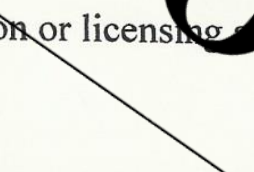

How strongly do you agree or disagree with each of the following statements?

24. You feel overwhelmed by paperwork.

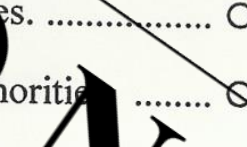
.. 0

O

0

$\circ$

O

Participant ID:-

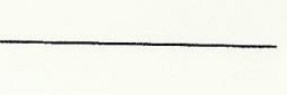

25. Your offices and equipment are adequate.

26. You have the skills needed to conduct effective outpatient counseling. O

27. Some staff get confused about the main goals for this program.

00

o

0 


\begin{tabular}{|ccccc|}
\hline $\begin{array}{l}\text { Disagree } \\
\text { Stronglv }\end{array}$ & Disagree & Uncertain & Agree & $\begin{array}{c}\text { Agree } \\
\text { Stronglv }\end{array}$ \\
\hline$(1)$ & $(2)$ & $(3)$ & $(4)$ & $(5)$ \\
\hline
\end{tabular}

32. Treatment planning decisions for clients here often have to be revised by a counselor supervisor.

$0 \quad 0$

33. Staff training and continuing education are priorities at this program.

34. Facilities here are adequate for conducting outpationt counseling.

35. You frequently are urknowledge of counselling wh be aff.

36. You were satisfied with the aning offered at worksheps available to y last year.

37. You used the Internet (Workd Wide We to communicate with other treatment professionals (e.g., list serves, bulletin boards, chat rooms) in the past month.

38. Management here fully trusts your professional judgment.

39. You feel appreciated for the job you do.

40. There is too much friction among staff members. O

41. Counselors at this program make a conscious effort to coordinate with other service professionals. O<smiles>[O]C[Si]</smiles>
O<smiles>[O]</smiles><smiles>[O]</smiles>

O

43. Staff generally regard you as a valuable source of information. O O $0 \quad 0 \quad 0$

\section{Ideas and suggestions from staff get fair
consideration by program management. \\ 42. Ideas and suggestions from staff get fair
consideration by program management. .... $\mathrm{O}$}

O

44. You have easy access for using the Internet at work. . 


\begin{tabular}{|ccccc|}
\hline $\begin{array}{l}\text { Disagree } \\
\text { Strongly }\end{array}$ & Disagree & Uncertain & Agree & $\begin{array}{c}\text { Agree } \\
\text { Stronglv }\end{array}$ \\
\hline (1) & $(2)$ & $(3)$ & (4) & (5) \\
\hline
\end{tabular}

45. The staff here always work together as a team. 0

46. Client assessments here are usually conducted using a computer. O

47. Your duties are clearly related to the goals of this prosram.

48. You learned new $\mathrm{jl}$ ls or techniques at a professional ony rence in the pastyes

49. You consistently plan head and carry outyour plan

50. You are under too many pressures to do your job effectively,

51. Counselors here are given broad authority in treating their own clients. 0 0

0

0

O 


\begin{tabular}{ccccc|}
$\begin{array}{l}\text { Disagree } \\
\text { Stronglv }\end{array}$ & Disagree & Uncertain & Agree & $\begin{array}{c}\text { Agree } \\
\text { Stronglv }\end{array}$ \\
\hline (1) & (2) & $(3)$ & $(4)$ & $(5)$ \\
\hline
\end{tabular}

60. You have enough opportunities to keep your counseling skills up-to-date.

61. Mutual trust and cooperation among staff in this program are strong.

62. Most client records here are computerized.

63. You are willing t new ideas even if some staff $\mathrm{m}$

64. Learning and using ne proc dures are easy foxyou.

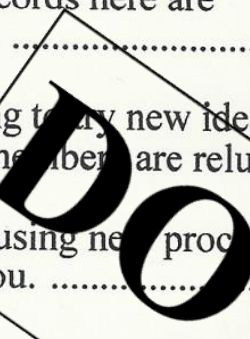

65. This program operates with clea/gc and objectives.

66. Staff members often show signs of stress and strain.

67. You feel like you aren't making a difference.

68. You usually accomplish whatever you set your mind on.<smiles>[CH2]O</smiles><smiles>O</smiles>

0

0

O 


\begin{tabular}{|ccccc|}
\hline $\begin{array}{l}\text { Disagree } \\
\text { Stronglv }\end{array}$ & Disagree & Uncertain & Agree & $\begin{array}{c}\text { Agree } \\
\text { Stronglv }\end{array}$ \\
\hline$(1)$ & $(2)$ & $(3)$ & $(4)$ & $(5)$ \\
\hline
\end{tabular}

74. Offices here allow the privacy

needed for individual counseling.

O

o

0

O

0

75. You are sometimes too cautious or slow to make changes.

76. Staff members are given too many rules here.<smiles>CCC(C)C</smiles>
0

77. You feel that it/s areal effert to come into work.

78. Counselors here der intervent ons together.

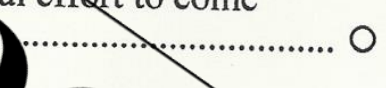

O

O

O

9. Program staff are always kept well informed.

80. The heavy workload here roduces program effectiveness.

81. You regularly read professional journal articles or books on child treatments.

82. You feel depressed.

83. Other staff often ask your advice about program procedures.

84. More open discussions about program issues are needed here. O

85. This program holds regular inservice training.

0

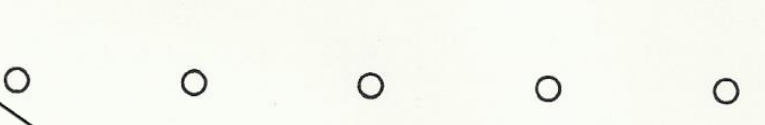

86. You give high value to the work you do here.

O

○

○

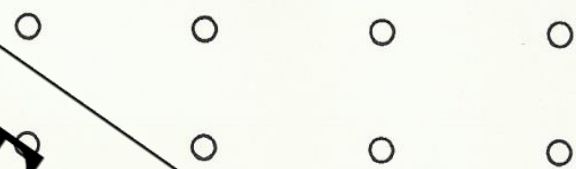

87. You frequently hear good staff ideas for improving treatment.

88. Other staff often ask for your opinions about counseling and treatment issues. 


\section{Disagree \\ Agree}

Stronglv Disagree Uncertain Agree Strongly

$\begin{array}{lllll}\text { (1) (2) (3) (4) } & \end{array}$

89. You are effective and confident

in doing your job.

.

90. You have a computer to use in your personal office space at work.

91. Everybody here does their fair share of work.

O

92. A larger support $\mathrm{staf}$ is needed to help meet program needs.

93. The genera/atti de ere is to ose new and changing chn of n................ 0

0

0

0

O

94. You do a good job of egul? updating andimprov

95. Staff members always feel frecto ask questions and express concerns in this program.

96. You have the skills needed to conduct effective individual counseling.

97. Staff frustration is common here.

98. You feel tired.

99. Management here has a clear plan for this program. O

100. You often influence the decisions of other staff here.

.

101. You are proud to tell others where you work.

102. You have convenient access to e-mail at work.

O

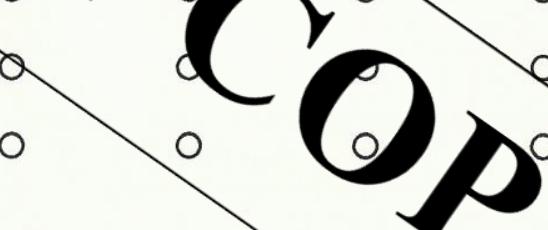

103. You are encouraged here to try new and different techniques. 


\begin{tabular}{|ccccc|}
\hline $\begin{array}{l}\text { Disagree } \\
\text { Stronglv }\end{array}$ & Disagree & Uncertain & Agree & $\begin{array}{c}\text { Agree } \\
\text { Stronglv }\end{array}$ \\
\hline (1) & (2) & (3) & (4) & (5) \\
\hline
\end{tabular}

104. You are able to adapt quickly when you have to shift focus.

$0 \quad 0$

000

105. You feel disillusioned and resentful.

O

O

O

0

106. You are viewed as a leader

by other staff here.

$0 \quad 0$

107. Computer equipment of this program is mostly old and outdated.

108. This program provid a $\mathrm{c}$ c nfortable reception/waiting ar fo

109. Staff here feel comfortable using computers.

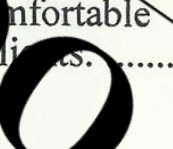
. .0

O

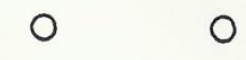

O

10. Frequent staff turnover is a problem for this program.

111. Counselors here are able to spend enough time with clients.

112. Support staff here have the skills they need to do their jobs.

113. Clinical staff here are well-trained. O

114. The director, counselors, and staff collaborate to make this program run effectively. O

115. More computers are needed in this program for staff to use. O

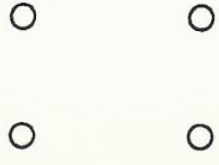

$0 \quad 0$

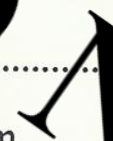
2 O

105 
118. In the last year, how often did you attend training workshops held within

50 miles of your agency?

\title{
None
}

\section{2}

34 or more

O

119. In the last year, how often did you attend training workshops held more than 50 miles from your agency? O

120. How many workshops do you expect to attend in the next 12 months? 0

121. In the last year, how many times did outside trainers come to your agency to give workshops? 0

122. In the last year, how many times did your agency offer special, in-house aining?

\author{
... $\mathrm{O}$
}

O
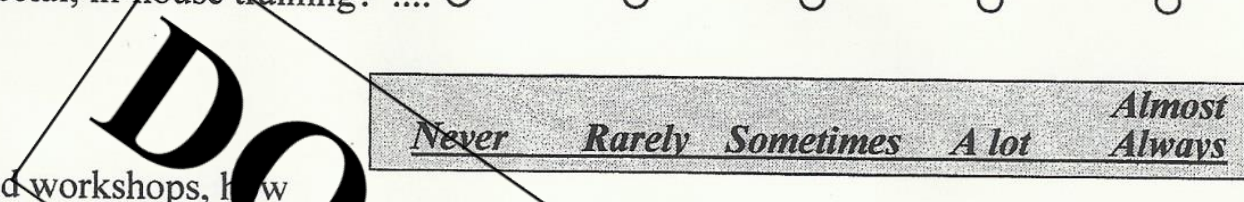

123. When you attend workshops,
often do you try out the new interventions or techniques learned?

124. Are your clients interested or tesponsive to new ideas or counseling materials when you try them?

125. In recent years, how often have you adopted (for regular use) new counseling interventions or techniques from a workshop?

126. When you have adopted new ideas into your counseling, how often have you encouraged other staff to try using them?

127. How often do new interventions or techniques that the staff from your program learn at workshops get adopted for general use? O O

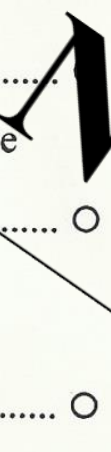

128. How often do new ideas learned from workshops get discussed or presented at your staff meetings?

129. How often does the management at your program recommend or support new ideas or techniques for use by all counselors? .... 
My program director -

\begin{tabular}{|ccccc|}
\hline $\begin{array}{l}\text { Disagree } \\
\text { Stronglv }\end{array}$ & Disagree Uncertain & Agree & $\begin{array}{c}\text { Agree } \\
\text { Stronglv }\end{array}$ \\
\hline$(1)$ & $(2)$ & $(3)$ & $(4)$ & $(5)$ \\
\hline
\end{tabular}

130. inspires others with his/her plans

for this facility for the future.

O

131. leads by example.

O

132. gets people to work together

for the same goal.

0

133. insists on only the best performance.

0

134. treats each of us as individuals with different needs, abilities, and aspirations.

135. takes time to listen aref discuss people's concerns.

136. encourages new waxs of lookirg at how we do our jobs.

137. gives special recognition to others' work when it is very good.

138. provides well-defined performance goals and objectives.

139. emphasizes using new ideas, services, administrative techniques, etc., before most other programs do. . 0

In the past year, you have -

140. invited someone in to help facilitate your sessions.

141. had colleagues observe your sessions. ○

142. received meaningful feedback on your performance from colleagues. ............ 0

143. visited other counselors' sessions.

144. received useful suggestions for counseling materials from colleagues. O 
How strongly do you agree or disagree with each of the following statements?

\begin{tabular}{|ccccc|}
\hline $\begin{array}{l}\text { Disagree } \\
\text { Strongly }\end{array}$ & Disagree Uncertain & Agree & Stronglv \\
\hline (1) & (2) & (3) & (4) & (5) \\
\hline
\end{tabular}

145. Many counselors in this program set high standards for themselves.

146. Counselors support the director in enforcing program policies and rules.

.

O

O

O

O

147. When making importary decisions,
the program always focuses on what's

147. When making importat decisions,
the program always focuses on what's best for client iphpro ament.

148. In the past year,
conversations with colle ues
the goals of this program

148. In the past year,
conversations with colle ues out
the goals of this program

148. In the past year,
conversations with colle ues out
the goals of this program

148. In the past year,
conversations with colle ues out
the goals of this program

148. In the past year,
conversations with colle ues out
the goals of this program here.

0

O

o

O

0

150. In the past year, you have had frequent conversations with colleagues about what helps clients improve.

151. Experienced counselors invite new counselors into their sessions to observe, give feedback, etc.
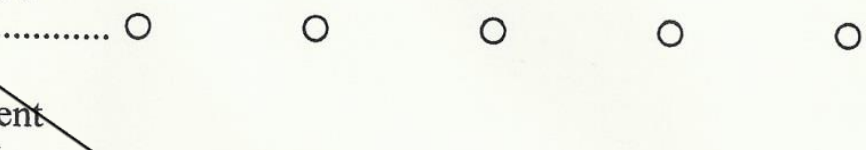


\begin{tabular}{|ccccc|}
\hline $\begin{array}{l}\text { Disagree } \\
\text { Stronglv }\end{array}$ & Disagree & Uncertain & Agree & $\begin{array}{c}\text { Agree } \\
\text { Strongly }\end{array}$ \\
\hline (1) & (2) & (3) & (4) & (5) \\
\hline
\end{tabular}

155. Our workday is organized to maximize counseling time.

O

$0 \quad 0$

$0 \quad 0$

156. This program sets high standards for client improvement. O

$0 \quad 0$

O

O

157. Many counselors in this program feel responsible to help each other do their best. O

158. Many counselors jin this program help maintain discipline in the entire 159. program, not just eir sessions. O

O

0

0

O

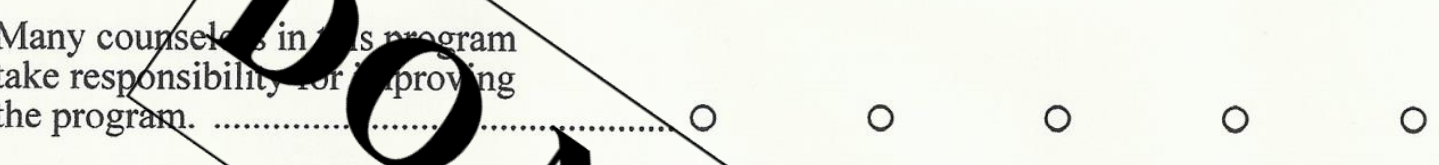
160. At this program, counselors worl
together to do what is "best for the clients."

161. This program has well-defined expectations for all clients.

162. Counselors talk about counseling in staff meetings, in the break room, etc.
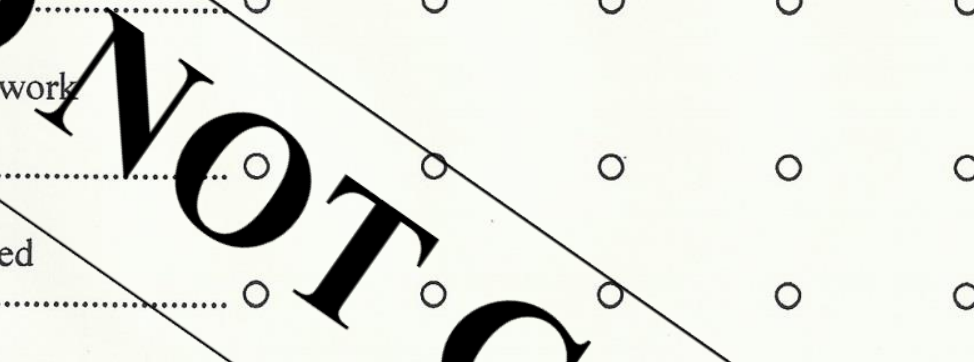


\section{Supervisor Background and Contact Information Form}

Please answer the following questions about your demographic information, your professional background, and your current job.

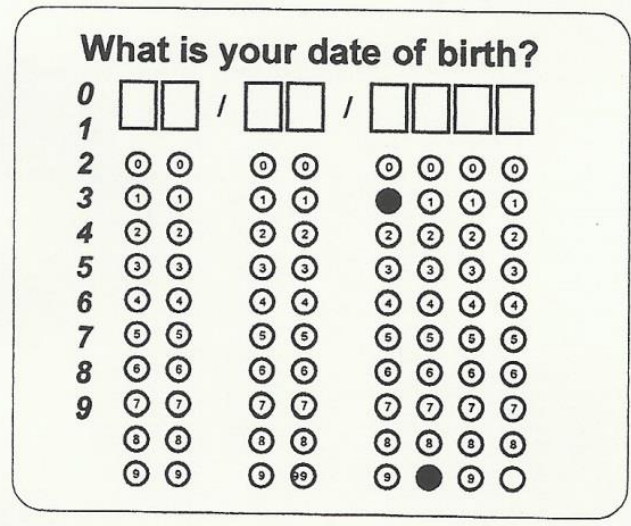

What is your Gender?

O Male

O Female

What is your Ethnicity?

O Hispanic or Latino

O Not Hispanic or Latino

What is your Race?

(Fill in all that apply)

O American Indian/Alaska Native

O Asian

O Native Hawaiian or other Pacific Islander

O Black or African American

OWhite

O More than one Race

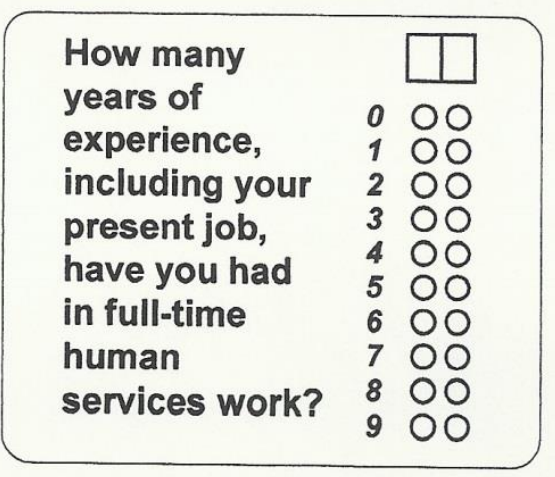

\begin{tabular}{|ll|}
\hline How many & $\square \square$ \\
years have & 000 \\
you worked & 100 \\
in your & 200 \\
present & 300 \\
agency? & 400 \\
& 500 \\
& 600 \\
& 700 \\
& 800 \\
& 900 \\
\hline
\end{tabular}

Your highest level degree is in:

What level of education
have you completed?

O High school graduate

O Some college

O Bachelors degree

O Some graduate work

O Masters degree

O Doctoral degree

Please Answer BOTH the

Ethnicity AND Race fields.
O Education

O Medicine

Nursing

O Psychology

O Social Work

O Other

What is your current role? (Fill in all that apply)

O Clinical Director

O Treatment Supervisor

O Program Director

O Other, please specify: 
Participant ID:

1. Are you professionally licensed (e.g., psychology or social work license?) O Yes O No Please indicate type of license: O LPC O LSW O LCSW O Psychologist O Other, please specify:

2. Setting where you do the majority of your work:

O Client's homes

O Group home/residential
O Your agency

Other, please specify:

3. Please rate your level of experience in the following areas:

For each of the following items, please fill-in one number to indicate your experience. Use the following scale:

$\begin{array}{ccccc}\begin{array}{c}\text { None } \\ \text { (Novice) }\end{array} & \text { Moderate } & & \begin{array}{c}\text { Substantial } \\ \text { (Expert) }\end{array} \\ 1 & 2 & 3 & 4 & 5\end{array}$

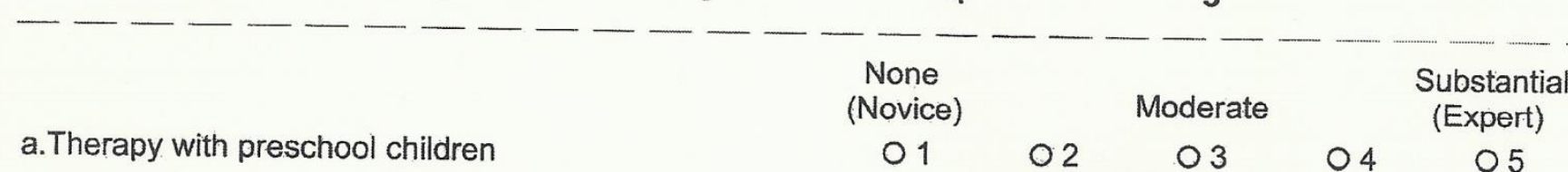
b. Assessment of child and family functioning
01
O 2
03
O 4
05

c. Cognitive Behavioral Therapy

01

02

03

04

05

d. Family Therapy

01

O 2

03

04

05

e. Behavioral Parent Training

01

02

03

O 4

05

4. In the last 6 months, what trainings have you attended? Please list the topics of any trainings here:

a.

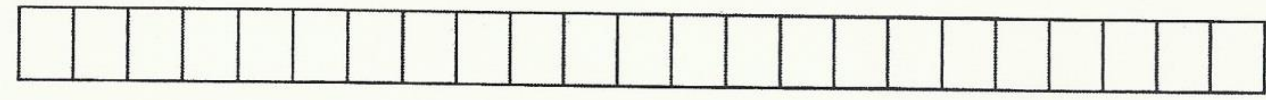

b.

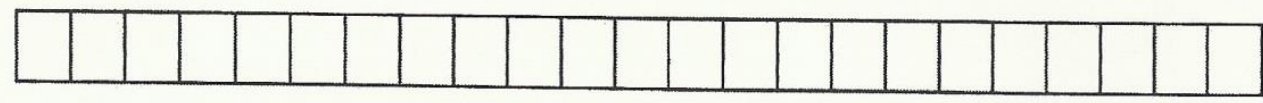

c.

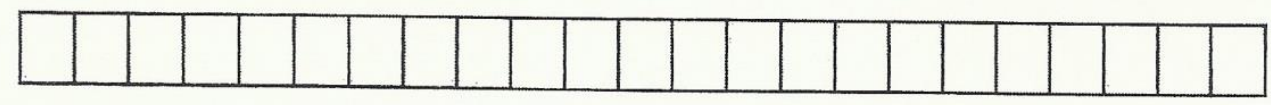

8. How long was each training (in hours)?
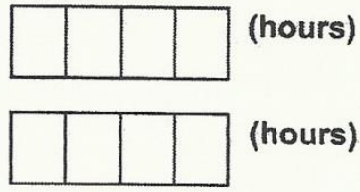

(hours) 
Participant 10:

\section{Please list your current Agency, Agency Program(s), Supervisor(s), Work Contact Information, Home Contact Information and Internet Access / Email Agency where you will be providing PCIT; \\ O Devereux Community Services \\ O Holcomb Behavioral Health Systems \\ O Child Guidance Resource Centers \\ O University Community Behavioral Health \\ O Aliernative Community Resource Program \\ O Nulton Diagnostic and Treatment Center}

\section{Agency Program(s):}

Primary Agency Program that you work in (Primary agency program should be the one that you do most of your work in) OBehavioral Health Rehabilitation Services / Wraparound O Family-Based O Inpatient O Outpatient

O Partial Hospitilization O School-Based O Other, please specify:

N/A Secondary Agency Program that you work in (Bubble "N/A" if you work in only one agency program)

O OBehavioral Health Rehabilitation Services / Wraparound O Family-Based O Inpatient O Outpatient

O Partial Hospitilization O School-Based O Other, please specify:

Supervisor(s): (Primary supervisor should be the direct supervisor with whom you have the most contact) Primary Supervisor's Name (first)

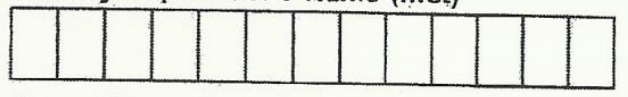

(last)

Does this person offer clinical or administrative oversight?
O Clinical
Administrative
O Both clinical and administrative

N/A Secondary Supervisor's Name (first)

$\mathrm{O}$

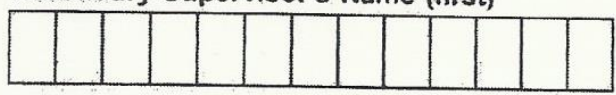

(last) (Bubble "N/A" if you only have 1 direct supervisor.)

Does this person offer clinical or administrative oversight?

Clinical $O$ Administrative $\bigcirc$ Both clinical and administrative

Work Contact Information:

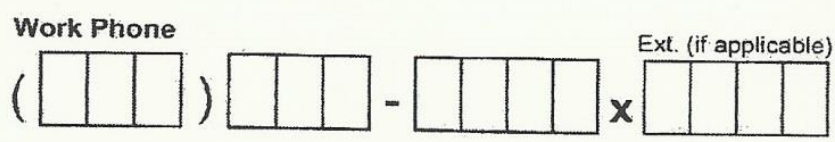

Work Email Address (ex. jsmith@companyname.com)
Work Cell Phone (if applicable)
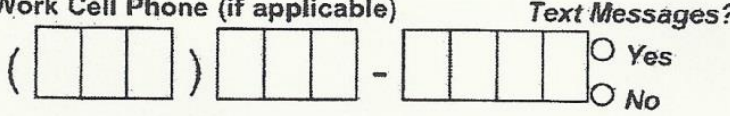

Work Pager (if applicable)

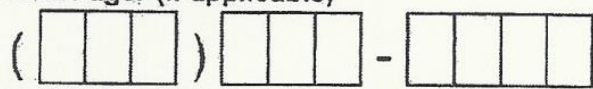


Participant ID

\section{Home Contact Information:}

Your Street Number \& Address

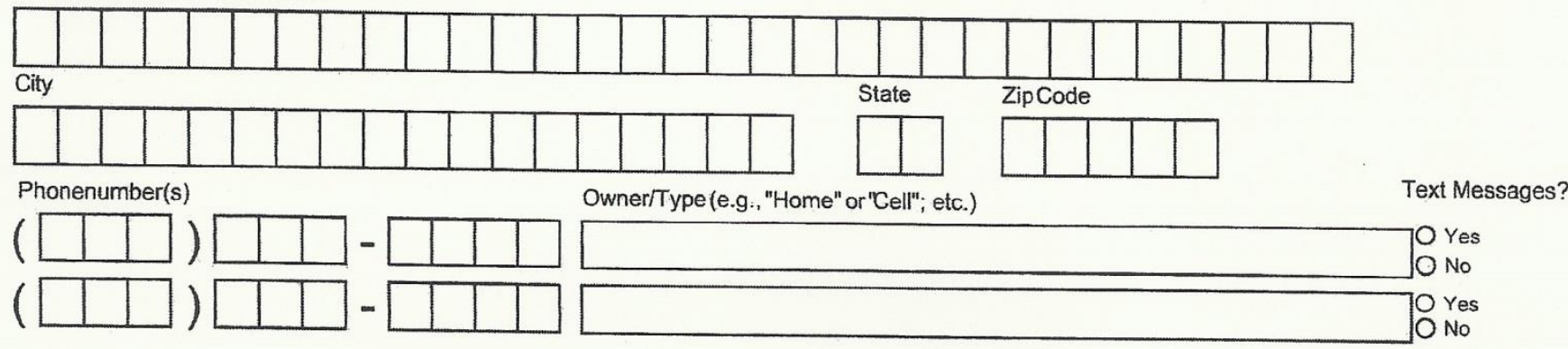

Internet Access / Email:

Do you have internet access at home? $\mathrm{O}$ Yes $\mathrm{O}$ No Do you have email access at home? $\mathrm{O}$ Yes

Email \#1: Address

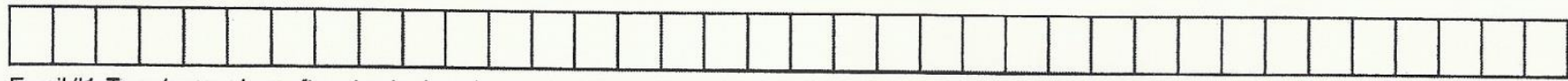

Email \#1: Type (owner, how often checked, work vs personal, confidentiality [who has access to account], etc...)

Email \#2: Address

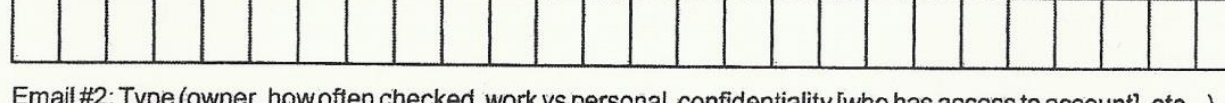

What is your preferred method of communication?

(Please check one)

O Email O Work Phone $O$ Cell Phone $O$ Home Phone O Text

Please provide information for another person who may help us contact you.

Person's Name (first)

(last)

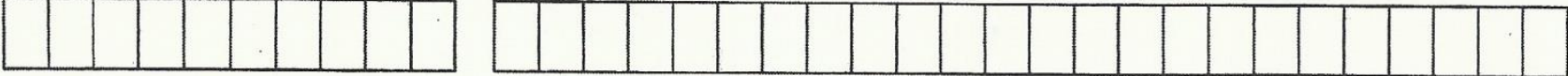

Number \& Street Address

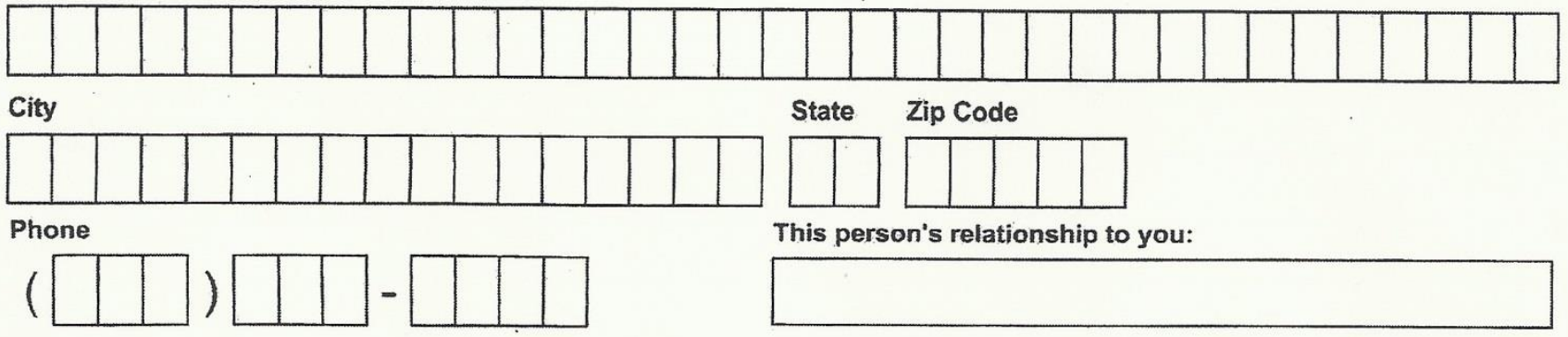


Participant ID:

\section{Supervisor Background and Contact Information Form (continued)}

If you have your own caseload, please answer questions 1 through 4:

1. How many clients are you currently treating (i.e., total number on your caseload)?

2. Considering all the clients on your caseload, what percentage fall into the following age ranges: (These totals should add up to be $100 \%$ )

\begin{tabular}{|l|l|}
\hline & Percentage $(\%)$ \\
\hline Under 3 years old & \\
\hline $3-5$ years old & \\
\hline $6-12$ years old & \\
\hline $13-18$ years old & \\
\hline Older than 18 years & \\
\hline
\end{tabular}

3. Considering all the clients on your caseload, what percentage of cases do you believe has had the following experiences? (some cases may have had multiple experiences, so the sum of all the percentages may exceed $100 \%$ )

\begin{tabular}{|l|l|}
\hline & Percentage (\%) \\
\hline Physical abuse/harsh physical discipline & \\
\hline Sexual abuse & \\
\hline Emotional abuse & \\
\hline Neglect & \\
\hline $\begin{array}{l}\text { Other child welfare concerns } \\
\text { please specify: }\end{array}$ & \\
\hline
\end{tabular}

4. Considering all the clients on your caseload, what percentage of cases do you believe has had the following diagnoses? (some cases may have had multiple diagnoses, so the sum of all the percentages may exceed $100 \%)$

\begin{tabular}{|l|l|}
\hline & Percentage (\%) \\
\hline Developmental delays & \\
\hline Autism spectrum Disorder & \\
\hline $\begin{array}{l}\text { Internalizing Disorders (e.g., anxiety, } \\
\text { depression) }\end{array}$ & \\
\hline $\begin{array}{l}\text { Externalizing Disorders (e.g., disruptive } \\
\text { behavior disorders, ADHD, Conduct Disorder) }\end{array}$ & \\
\hline Learning Disorders & \\
\hline
\end{tabular}

5. What is the primary service area for your organization? (please check one)

$\square$ Rural

$\square$ Suburban

$\square$ Urban 
Participant ID:

6. Does your organization primarily serve?

\begin{tabular}{lll}
\hline Adults & Yes & No \\
\hline Adolescents & Yes & No \\
\hline Young children & Yes & No \\
\hline Clients involved with child welfare & Yes & No \\
\hline Clients involved with criminal justice & Yes & No \\
\hline
\end{tabular}

7. Are you employed part-time or full-time at your agency? (please check one)

$\square$ Full-time

$\square$ Part-time

a. If full-time, what is your annual salary?

b. If part-time, what is the hourly rate you get paid?
Please circle yes or no for each

No

No 


\section{Agency Staff Change Form - PCIT ACROSS PA \\ Self-Report Version \\ For Supervisors}

It is important for us to know about any changes in how and/or where you work. We are interested in any changes since the last time you completed an assessment for PCIT Across PA.

Have you been employed at the same agency since your last assessment?

IF NO,

What date did you leave your Agency/Organization?

What is the name of the new agency/organization you work for?

What type of agency/organization is it? (pick the one that best describes it)

- Private practice

- Community Mental Health Center

- Hospital System

- Other (please specify):

Is the new agency/organization in the PCIT Across PA study? Yes No

What program or service line with the new agency/organization are you now working in? (pick all that apply)

- Outpatient

- BHRS

- Family-based

- Other (please specify):

What is your role in the new organization? (pick all that apply)

- Clinician

- Supervisor

- Program Manager

- Director

- Other (please specify):

Have you changed your program (service line) within your Agency/Organization?

If YES,

What date?

What is your new program (service line)? (pick all that apply)

- Outpatient

- BHRS

- Family-based

- Other (please specify):

\section{Do you have a new role (job type) within your Agency/Organization?}

IF YES,

What date did you change your role (job type?)

What is your new role (job type)? (pick all that apply)

- Clinician

- Supervisor 
- Program Manager

- Director

- Other (please specify):

Have you had a change in the number of hours your work (moving from part-time to full-time or vice versa)? Yes No

What date did your change in hours take effect?

Please describe the change in hours (pick one)

- Decrease in Hours

- Increase in Hours

Have you taken a leave of absence (e.g., maternity leave, FMLA, etc....) since your last assessment? Yes No

IF YES,

What date did your leave begin?

Please describe the reason for your leave:

Have you returned to work from a leave of absence (e.g., maternity leave, FMLA, etc...) since your last assessment? Yes No

IF YES,

What date did you return to work?

Please describe the reason for your leave: 


\section{Agency Staff Change Form - PCIT ACROSS PA \\ Self-Report Version \\ For Administrators}

It is important for us to know about any changes in how and/or where you work. We are interested in any changes since the last time you completed an assessment for PCIT Across PA.

Have you been employed at the same agency since your last assessment?

IF NO,

What date did you leave your Agency/Organization?

What is the name of the new agency/organization you work for?

What type of agency/organization is it? (pick the one that best describes it)

- Private practice

- Community Mental Health Center

- Hospital System

- Other (please specify):

Is the new agency/organization in the PCIT Across PA study? Yes No

What program or service line with the new agency/organization are you now working in? (pick all that apply)

- Outpatient

- BHRS

- Family-based

- Other (please specify):

What is your role in the new organization? (pick all that apply)

- Clinician

- Supervisor

- Program Manager

- Director

- Other (please specify):

Have you changed your program (service line) within your Agency/Organization?

If YES,

What date?

What is your new program (service line)? (pick all that apply)

- Outpatient

- BHRS

- Family-based

- Other (please specify):

Do you have a new role (job type) within your Agency/Organization?

IF YES,

What date did you change your role (job type?)

What is your new role (job type)? (pick all that apply)

- Clinician

- Supervisor 
- Program Manager

- Director

- Other (please specify):

Have you had a change in the number of hours your work (moving from part-time to full-time or vice versa)? Yes No

What date did your change in hours take effect?

Please describe the change in hours (pick one)

- Decrease in Hours

- Increase in Hours

Have you taken a leave of absence (e.g., maternity leave, FMLA, etc...) since your last assessment? Yes No

IF YES,

What date did your leave begin?

Please describe the reason for your leave:

Have you returned to work from a leave of absence (e.g., maternity leave, FMLA, etc...) since your last assessment? Yes No

IF YES,

What date did you return to work?

Please describe the reason for your leave: 


\section{Organizational Readiness for Change (TCU ORC) Treatment Director Version (TCU ORC-D) Instruction Page}

This survey asks questions about how you see yourself as a program director or counseling supervisor, and how you see your program. It begins on the next page with a short demographic section that is for descriptive purposes only. The Anonymous Linkage Code is requested so that information you give now can be "linked" to your responses to similar questions you may be asked later.

To complete the form, pyease mork your answers by completely filling in the appropriate circles. If you do not feel comf ay giving an answer to a particular statement, you may skip it and move on to the nexts em to If an iten does not apply to you or your workplace, leave it blank. PLEASEDO D P RMS. The examples below show how to mark the circles -

Person 1. I like chocolate ice cream.
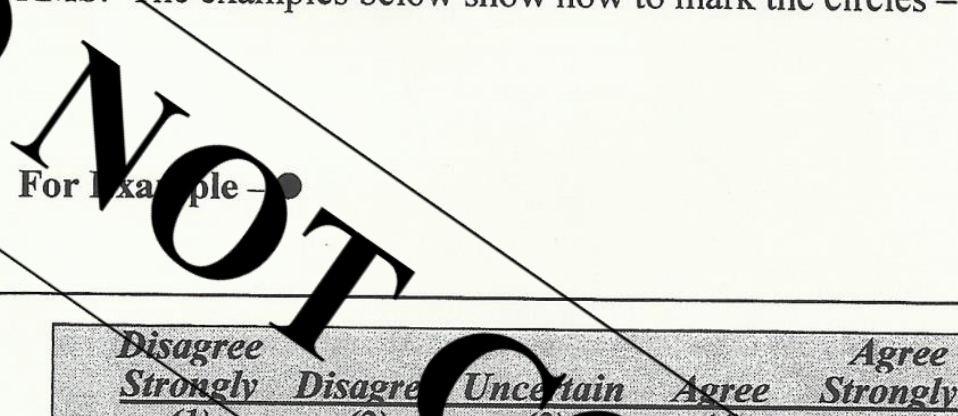

(1)

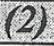

This person disagrees a little so she probably doesn't like chocolate ice cream

Person 2. I like chocolate ice cream. ○

This person likes chocolate ice cream a lot.

Person 3. I like chocolate ice cream. O O ○

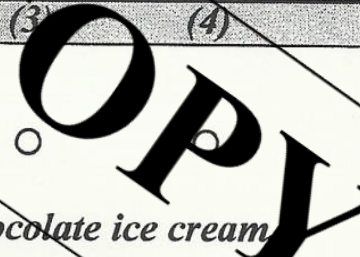

This person is not sure if he likes chocolate ice cream or not. 
FOR MUIMISTRATIEPURPOSES

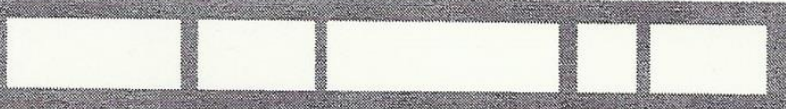

\section{Organizational Readiness for Change (TCU ORC) \\ Treatment Director Version (TCU ORC-D)}

PLEASE FILL IN THE CIRCLE THAT SHOWS YOUR ANSWER TO EACH ITEM.

\begin{tabular}{|ccccc|}
\hline $\begin{array}{l}\text { Disagree } \\
\text { Strongly }\end{array}$ & Disagree & Uncertain & Agree & $\begin{array}{c}\text { Agree } \\
\text { Stronglv }\end{array}$ \\
\hline$(1)$ & $(2)$ & $(3)$ & $(4)$ & $(5)$ \\
\hline
\end{tabular}

Your program needs additional guidance in -

1. documenting service needs of clients for making treatment placements.

2. tracking and evaluatir perfornance of clients over tiphe.

3. obtaining informative program effectiveness.

4. automating client records for billing and financial applications.

5. evaluating staff performance and organizational functioning.

6. selecting new treatment interventions and strategies for which staff need training.

7. improving the recording and retrieval of financial information.

8. generating timely "management" reports on clinical, financial, and outcome data.

Your counseling staff needs more training for -

9. assessing client problems and needs. .

10. increasing client participation in treatment. . O 0<smiles>O=C1CCCC1</smiles> 


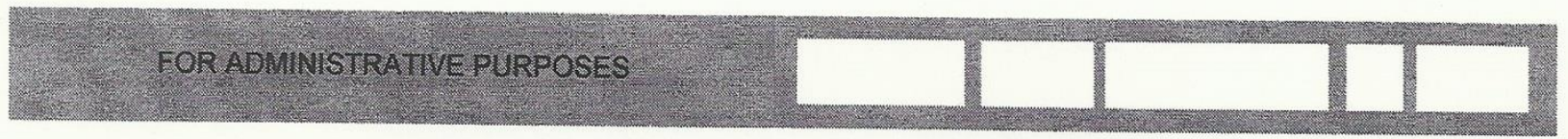

\begin{tabular}{|ccccc|}
\hline $\begin{array}{l}\text { Disagree } \\
\text { Strongly }\end{array}$ & Disagree & Uncertain & Agree & $\begin{array}{c}\text { Agree } \\
\text { Stronglv }\end{array}$ \\
\hline$(1)$ & $(2)$ & $(3)$ & $(4)$ & $(5)$ \\
\hline
\end{tabular}

15. improving cognitive focus of clients during group counseling. 0

16. using computerized client assessments. ..... $\bigcirc$

\section{Current pressures to make} program changes come from -

17. clients in the program

18. program staff phembexs.

19. program sypery

20

21. community action groups or manzgers.<smiles>O[C@H]1C[C@@H]2CO[C@H]1O2</smiles>
O

22. funding and oversight agencied

23. accreditation or licensing authorities

How strongly do you agree or disagree with each of the following statements?

24. Your staff prefer training content that is based on scientific evidence.

25. Your offices and equipment are adequate.

26. You have the skills to conduct effective staff meetings.

O

27. Some staff get confused about the main goals for this program. O $0 \quad 0 \quad 0$ $0 \quad 0$<smiles>[O-]</smiles>

O<smiles>O=C1CCCO1</smiles>
$\begin{array}{llll}0 & 0 & 0 & 0\end{array}$
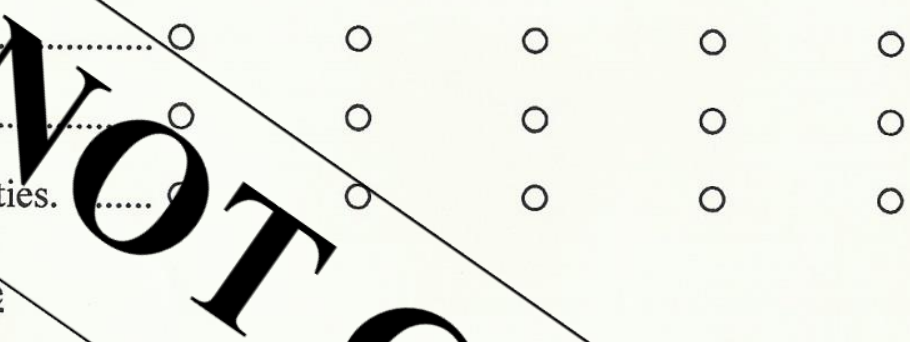

28. Staff here all get along very well.

29. Psychodynamic theory is commonly used in counseling here. 


\section{FORADUINISTRATIVE PURPOSES}

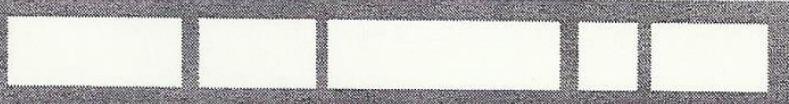

\section{Disagree}

Stronglv

(I)

D

Disagree $U$

(2)

Incertain

Agree

Agree

Strongly

(5)

30. Your staff often has trouble implementing

concepts they learn at conferences. .

31. Program staff understand how this program fits as part of the treatment system in your community. O

32. Treatment planning decisions for clients here often have to be revised by a counselor supervisor.

33. Staff training and continulin are priorities at this progra

34. Offices here are adequate for conducting group counseling.

35. You frequently discuss new counseling ideas with staff.

36. You were satisfied with outside training available to your staff last year.

37. You used the Internet (World Wide Web) to communicate with other treatment professionals (e.g., list serves, bulletin boards, chat rooms) in the past month.<smiles>[CH]O</smiles><smiles>OC1CCC1</smiles>

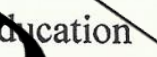
eling<smiles>OCCO</smiles><smiles>O=C1CCCC1</smiles><smiles>O[14CH2]O[14CH2]O[14CH2]O</smiles>

38. You fully trust the professional judgment of staff who work with clients here.

39. Pharmacotherapy and medications are important parts of this program.

40. There is too much friction among staff members.

41. Some staff members here resist any type of change. O

42. You always listen to ideas and suggestions from staff. 


\section{FOR ADMMISTRATIVE PURPOSES}

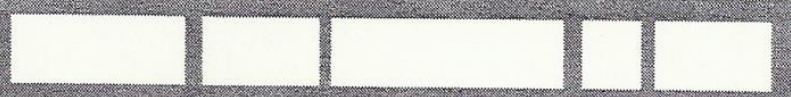

\section{Disagree \\ Strongl \\ (1) \\ Agree \\ Strongly \\ (5)}

43. Staff generally regard you as a valuable source of information.

O

O

O

O

O

44. You have easy access for using the Internet at work.

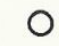

0

O

O

45. The staff here always works together as a team.<smiles>O</smiles>

0

0

O

46. Client assessments here ar usualy conducted using a conpy

47. Your duties are clearly lat the goals of this rogram.

48. You learned new manasement skirs or techniques at a professiona conference in the past year.

49. You consistently plan ahead and carry out your plans.

50. You are under too many pressures to do your job effectively.

51. Counselors here are given broad authority in treating their own clients.

52. This program encourages and supports professional growth. .0

53. Behavior modification (contingency management) is used with many clients here. O

4. You read about new techniques and treatment information each month. O (

Staff here are always quick to help one another when needed. O

56. Computer problems are usually repaired promptly at this program. 


\section{FOR ADUINISTRATIVE PURPOSES}

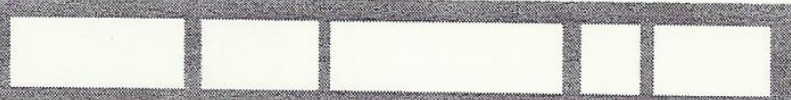

\section{Disagree Agree \\ Stronglv Disagree Uncertain Agree Stronglv

(1) (2) (3) (4) (5)

57. Novel treatment ideas by staff are discouraged.

. 0

O

0

0

O

58. There are enough counselors here to meet current client needs.

59. The budget here Allows staff to attend professional conferenses each year.

60. You have exour op portunities to keep your managew it s' ls un-to-date.

61. Mutual xust and coo ratio among staff in this program are strkng

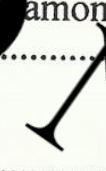

62. Most client records here are computerized.

63. You are willing to try new idess even if some staff members are reluctant.

64. Learning and using new procedures are easy for you.

65. This program operates with clear goals and objectives.

O

$0 \quad 0 \quad 0$

66. Staff members often show signs of stress and strain. O

67. You have staff meetings weekly.

68. You usually accomplish whatever you set your mind on.

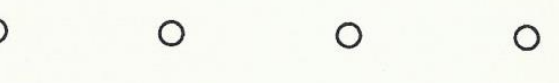




\section{FOR ADMINISTRATIVE PURPOSES}

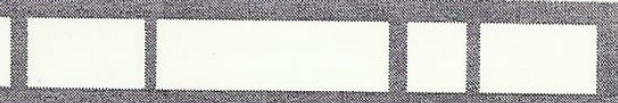

\section{Disagree}

Stronalv

(1)

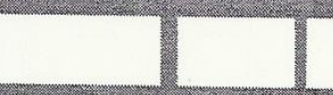

1)

\begin{tabular}{cccc} 
Disagree Uncertain & Agree & $\begin{array}{c}\text { Agree } \\
\text { Stronglv }\end{array}$ \\
\hline$(2)$ & $(3)$ & $(4)$ & $(5)$
\end{tabular}

72. The formal and informal communication channels here work very well.<smiles>[CH]</smiles><smiles>[131I-]</smiles>

73. You have program policies that limit staff access to the Internet and use of e-mail.

74. Offices here allow the privacy needed for individual counseling.

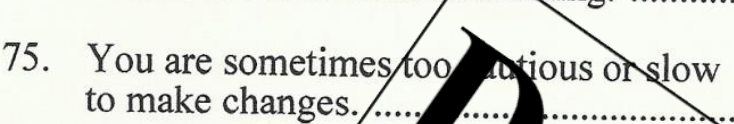

76. Staff members think they h too many rules here.

77. You feel a lot of stress here.

78. 12-step theory (AA/NA) is followed by many of the counselors here.

79. Program staff are always kept well informed.

80. The heavy workload here reduces program effectiveness.

81. You regularly read professional journal articles or books on drug abuse treatment.

82. Communications with other programs that have similar interests would help.

83. Staff readily implement your ideas for changing program procedures.

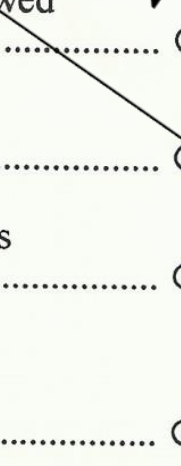<smiles>[14CH3]</smiles>

(1)

O

O

$\begin{array}{lllll}0 & 0 & 0 & 0 & 0\end{array}$




\section{FOR ADMINISTRATIVE PURPOSES}

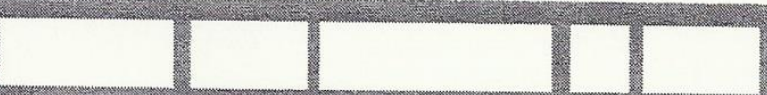

\section{Disagree}

Strongly

(1)

Agree

sagree Uncertain Agree

(4)

Strongly.

(5)

87. You frequently hear good staff ideas

for improving treatment.

O

O

0

O

88. Staff seek your opinions about counseling and treatment issues.

$\begin{array}{lllll}0 & 0 & 0 & 0 & 0\end{array}$

89. You are effective and confident

in doing your job.

90. You have a cophpy to personal office space a

91. Some staff here do not do of work.

92. A larger support staff is meeded to help meet program needs.

93. The general attitude here is to use new and changing technology.

94. You do a good job of regularly updating and improving your skills.

95. Staff members always feel free to ask questions and express concerns in this program.

96. You are highly effective in working with community leaders and board members.

97. Staff frustrations are common here.

98. Direct access to counseling resources on the Internet is needed by staff here.<smiles>O</smiles>

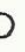




\section{FOR ADUINISTRATIVE PURPOSES}

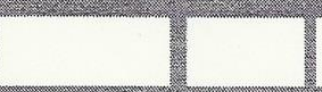

Disagree

Strongl

(I)

$\begin{array}{cccc}\text { Disagree Uncertain } & \text { Agree } & \begin{array}{c}\text { Agree } \\ \text { Strongly }\end{array} \\ (2) & (3) & \text { (4) } & (5)\end{array}$

102. You have convenient access to e-mail at work.

.

103. You encourage counselors to try new and different/chniques.

104. You are able tø ad quidt quekly when you have

105. Cognitive theor guides much of the co seliy
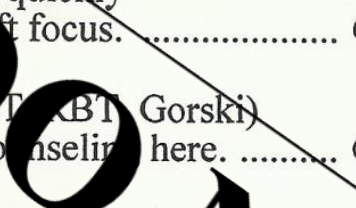

O

Gorski here.

106. You are viewedas a strong leader by the staff here.

107. Computer equipment at this program is mostly old and outdated.

108. This program provides a comfortable reception/waiting area for clients.

109. Staff here feel comfortable using computers.

110. Frequent staff turnover is a problem for this program.

111. Counselors here are able to spend enough time with clients.

O

$\mathrm{O}$

112. Support staff here have the skills they need to do their jobs.

0

$\mathrm{O}$

0

0

113. Clinical staff here are well-trained.

O

$\mathrm{O}$

O

$0 \quad 0$

114. The workload and pressures at your program

keep motivation for new training low. ....... $\mathrm{O}$

O $\quad 0$

0

O

115. More computers are needed in

this program for staff to use.

O

$0 \quad 0$

$0 \quad 0$

O 